\title{
Álgebras de Koszul Inclinadas
}

\author{
Regina Maria de Aquino
}

TESE APRESENTADA

$$
\mathrm{AO}
$$

INSTITUTO DE MATEMÁTICA E ESTATÍSTICA

DA

UNIVERSIDADE DE SÃO PAULO

PARA

OBTENÇÃO DO GRAU DE DOUTOR

EM

MATEMÁTICA

\section{Área de concentração: Álgebra Orientador: Prof. Dr. Eduardo do Nascimento Marcos}

Durante parte da elaboração deste trabalho a autora recebeu apoio financeiro do CAPES

-São Paulo, dezembro de 1998- 


\title{
ÁLGEBRAS DE KOSZUL INCLINADAS
}

\author{
Este exemplar corresponde à redação \\ final da tese de doutoramento, devida \\ mente corrigida e defendida por \\ Regina Maria de Aquino e \\ aprovada pela comissão julgadora.
}

São Paulo, 23 de março de 1999.

Banca Examinadora:

Prof.Dr. Eduardo do Nascimento Marcos - IME/USP

Prof.Dra. Maria Izabel Ramalho Martins - IME/USP

Prof.Dr. Paulo Brumatti - IMECC/UNICAMP

Prof.Dr. Arnaldo Garcia - IMPA/CNPq

Prof.Dr. Norai Romeu Rocco - IM/UnB 


\section{Dedicatória}

À minha mãe, minhas irmãs e meu querido filho André.

"É na adversidade que se constrói a força do caráter.

E na amizade, o coração generoso." 


\section{Agradecimentos}

Um coração grato, como sinto o meu, agora, é capaz de abraçar o mundo. Afinal, nenhum trabalho está posto, exclusivamente, em sua culminância, mas resulta de um esforço cotidiano no qual muitos compartilham. Agradeço à cada um que tenha dado sua contribuição nesta minha tarefa, mas preciso destacar alguns destes.

Agradeço mais especialmente:

À minha mãe, Hellanice, e às minhas irmãs, Sonia e Tania, pelo apoio material e emocional, sem os quais este trabalho não se completaria.

Ao meu filho, André, pela paciência e tranquilidade que me ofereceu ao enfrentar momentos tão tumultuados, como os que tivemos.

Ao meu orientador, Prof. Dr. Eduardo Marcos, pela dedicação constante.

Ao Prof. Dr. E.L. Green, pelo apoio e co-orientação durante meu estágio na Universidade Estadual de Virginia, USA.

Ao CAPES, que financiou este estágio.

Aos professores Héctor, Flávio e Bel pelo constante apoio acadêmico.

À Joelma, por seu auxílio inestimável na digitação deste trabalho.

Aos amigos Regina e Mário, Zita e Cau, Marta e André, Hamilton, Luciano, Maisa e Valquiria, que tanto me ajudaram nas dificuldades cotidianas.

Aos amigos em Blacksburg : Paulo, Marília e Mike, Ana Claudia, Tel, Craig e Adeel, que tornaram mais suave a estadia num país estranho.

A Cris , Jorge e Roseli, companheiros de trabalho sempre presentes.

Aos amigos, que sempre me trouxeram palavras de encorajamento.

Grata por tudo que recebi de vocês! 


\section{Resumo}

Sejam $\Lambda$ uma $k$-álgebra de dimensão finita sobre o corpo $k, T$ um $\Lambda$ módulo inclinante e $\Gamma=\operatorname{End}_{\Lambda}(T)$, o anel de endomorfismo de $T$ sobre $\Lambda$.

Através da caracterização dos morfismos, entre os somandos diretos de $T$ estabelecemos um critério que permite decidir quando a álgebra inclinada graduada $\Gamma \cong k Q / I_{I}$, onde $I$ um ideal graduado, é uma álgebra de Koszul.

Seja $\Gamma$ uma $k$-álgebra $\mathbb{Z}$-graduada, 1-gerada e de decomposição básica. Então, temos que $\Gamma$ é quadrática se e somente se vale que:

$\operatorname{dim}_{k} \operatorname{Hom}_{\Lambda}\left(I / I^{2}, \Gamma / r\right)-\operatorname{dim}_{k} \operatorname{Hom}_{\Lambda}\left(r P_{(1)}, \Gamma / r\right)+\operatorname{dim}_{k} \operatorname{Hom}_{\Lambda}\left(r^{2}, \Gamma / r\right)=0$, onde $r$ é o radical graduado de Jacobson de $\Gamma, I$ o ideal de relações e $P_{(1)}$ a cobertura projetiva de $\Omega^{1}(\Gamma / r)$.

Provamos que as álgebras quadráticas de dimensão global 3 e tais que pd $r^{2} \leq 2=\operatorname{pd} \frac{r}{r^{2}}$ são álgebras de Koszul se, e somente se, $r^{2}$ é um módulo de Koszul.

Seja $\mathcal{L}(\Gamma)$ a classe dos $\Gamma$-módulos com apresentação linear e $\mathcal{K}(\Gamma)$ a classe dos $\Gamma$-módulos que sejam módulos de Koszul. Se $\Gamma$ é uma álgebra de Koszul de dimensão global 2, então, temos que, em geral, as classes de módulos $\mathcal{L}(\Gamma)$ e $\mathcal{K}(\Gamma)$ não coincidem.

Seja $\Gamma$ uma álgebra Brenner-Butler inclinada. Então $\Gamma$ é uma álgebra de Koszul e $\mathcal{L}(\Gamma)=\mathcal{K}(\Gamma)$. Também, apresentamos uma descrição completa da classe $\mathcal{K}(\Gamma)$, e mostramos que, neste caso, esta classe pode ser infinita. 


\begin{abstract}
Let $\Lambda$ be a $k$-algebra finite dimensional over a field $k, T$ be a tilting $\Lambda$-module and $\Gamma=\operatorname{End}_{\Lambda}(T)$, the endomorphism ring of $T$ over $\Lambda$.

Throughout the caracterization of morphisms between the direct summands of $T$, we obtained a criterion to decide when the tilting algebra $\Gamma \cong k Q / I$, with $I$ a grade ideal, is a Koszul algebra.
\end{abstract}

Consider a $\mathbb{Z}$-graded $k$-algebra $\Gamma, 1$-generated, split basic. We proved that $\Gamma$ is a quadratic algebra if only if we have that

$\operatorname{dim}_{k} \operatorname{Hom}_{\Lambda}\left(I / I^{2}, \Gamma / r\right)-\operatorname{dim}_{k} \operatorname{Hom}_{\Lambda}\left(r P_{(1)}, \Gamma / r\right)+\operatorname{dim}_{k} \operatorname{Hom}_{\Lambda}\left(r^{2}, \Gamma / r\right)=0$ where $r$ is the graded Jacobson radical of $\Gamma, I$ is the ideal of relations and $P_{(1)}$ is the projective cover of $\Omega^{1}(\Gamma / r)$.

We proved that the quadratic algebras with global dimension 3 and such that pd $r^{2} \leq 2=\operatorname{pd} \frac{r}{r^{2}}$, are Koszul algebras if only if $r^{2}$ is a Koszul module.

Let $\mathcal{L}(\Gamma)$ be the class of $\Gamma$-modules with linear presentation and $\mathcal{K}(\Gamma)$ the class of Koszul $\Gamma$-modules. If $\Gamma$ is a Koszul algebra with global dimension 2, then we have that $\mathcal{L}(\Gamma)$ and $\mathcal{K}(\Gamma)$ are not coincidents, in general.

We proved that if $\Gamma$ is a Brenner-Butler tilting algebra then it is a Koszul algebra and $\mathcal{L}(\Gamma)=\mathcal{K}(\Gamma)$. Also, we gave a complete description about $\mathcal{K}(\Gamma)$, that could be infinite, in that case. 


\section{Sumário}

Introdução

Capítulo 1 - Preliminares

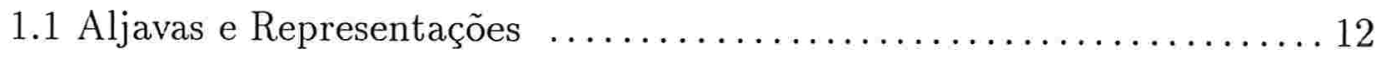

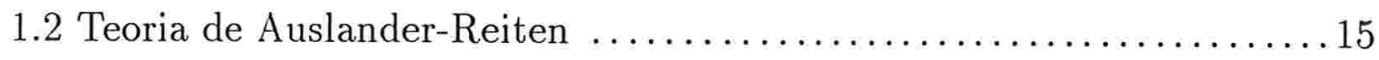

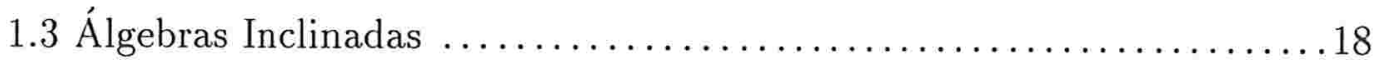

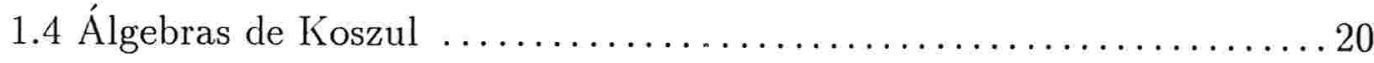

Capítulo 2 - Sobre Álgebras de Kozsul com dimensão global finita

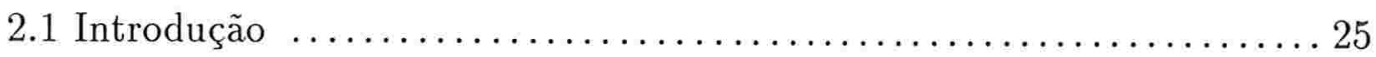

2.2 Álgebras quadráticas de dimensão global finita . . . . . . . . . . . . 27

2.3 Módulos de Koszul sobre álgebras de Koszul de dimensão global 2 . . 38

Capítulo 3 - Álgebras de Koszul Brenner-Butler-inclinadas

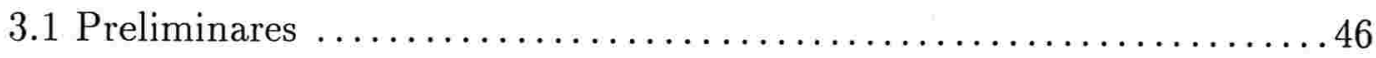

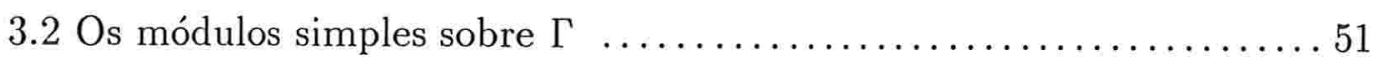

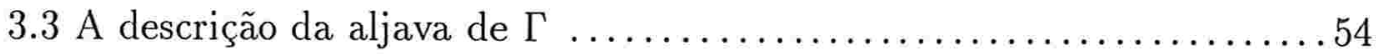

3.4 Álgebras Brenner-Butler-Koszul . . . . . . . . . . . . . . . . . . 59

3.5 Os módulos de Koszul sobre as álgebras BB-inclinadas . .........66 66

3.6 Uma generalização para as álgebras $B B$-inclinadas $\ldots \ldots \ldots \ldots \ldots 75$

Capítulo 4 - Álgebras inclinadas graduadas

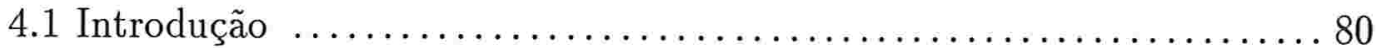

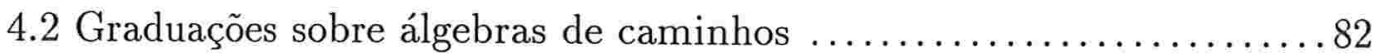

4.3 A graduação induzida por morfismos homogêneos .............. 84

$4.4 \mathrm{~A}$ apresentação projetiva minimal de $\Gamma / r \ldots \ldots \ldots \ldots \ldots \ldots \ldots$

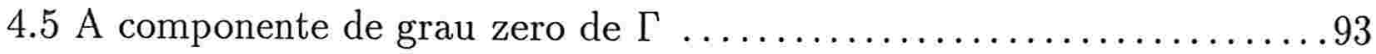




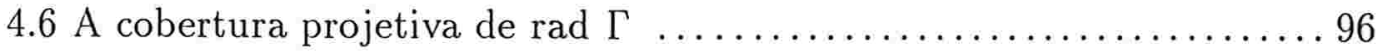

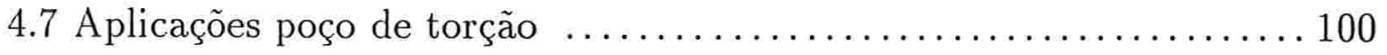

4.8 Álgebras de Koszul inclinadas ............................ 104

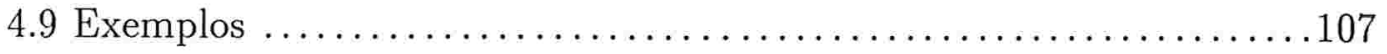

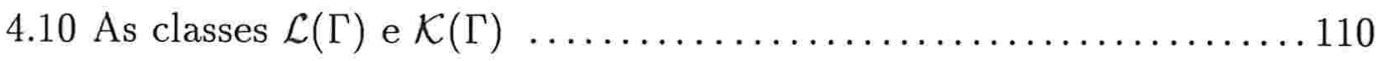

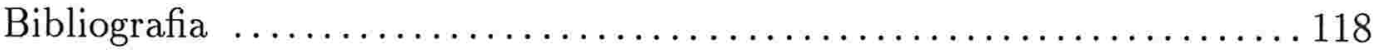




\section{Introdução}

As álgebras de Koszul têm tido muitas aplicações em vários campos da matemática, como por exemplo, na álgebra comutativa e topologia algébrica. Recentemente, foram obtidas importantes aplicações de álgebras de Koszul não-comutativa à teoria de Lie, à topologia algébrica e aos grupos quânticos. Assim, se torna uma questão interessante identificar as álgebras, ou classes de álgebras, que são álgebras de Koszul.

Por outro lado, temos a teoria das álgebras inclinadas que, de certa forma, generaliza a equivalência de Morita, quando nos traz informações da classe de módulos de uma álgebra, através da classe de módulos da álgebra hereditária, da qual esta se originou.

Este trabalho tem como objetivo relacionar essas duas classes de álgebras, no sentido de identificar quais são as álgebras inclinadas que sejam, também, álgebras de Koszul. Uma vez estabelecida esta identificação, ganhamos em informação, quando se tratar de buscar os módulos sobre essa álgebra Koszulinclinada, que sejam módulos de Koszul, já que o estudo da categoria de módulos desta vai poder se dar através do estudo da categoria dos módulos da álgebra hereditária inicial.

Numa situação inicial consideramos $\Lambda$ uma $k$-álgebra de dimensão finita sobre um corpo $k$, hereditária, básica, indecomponível, e $T$ um $\Lambda$-módulo à esquerda, inclinante. Nesta situação, o anel de endomorfismo $\Gamma=\operatorname{End}_{\Lambda}(T)$ é chamado de álgebra inclinada (cf. [HR]). Pelos resultados apresentados em $[\mathrm{AS}, 1]$, por I. Assem, vale que gldim $\Gamma=2$.

As perguntas iniciais que motivaram este trabalho foram as seguintes:

(1ํㅡ) Quando $\Gamma$ é uma álgebra de Koszul? 
(2aㅡ) Qual o grafo subjacente à álgebra $\Gamma$ ?

(3a) Como é a classe dos módulos de torção de $\Lambda$-mod que produzem módulos de Koszul sobre $\Gamma$ ?

Para responder nossa primeira pergunta, precisamos decidir quando estas álgebras são quadráticas, pois, de acordo com os resultados apresentados por Green e Martinez-Villa, em [GM,1], os conceitos Koszul e quadrática são equivalentes sobre álgebras de dimensão global 2. Um estudo mais atento em $[R]$, mostra que, mesmo no caso em que os módulos inclinantes são preprojetivos, não ocorre que $\Gamma=\operatorname{End}_{\Lambda}(T)$ é uma álgebra quadrática. Além disso, ocorre, também, que o ideal da apresentação de $\Gamma$ não é um ideal homogêneo, em geral.

Trabalhando no sentido de responder a primeira pergunta, conseguimos uma outra caracterização de álgebras quadráticas graduadas, (cf. Teorema 2.3), que nos levou a obter como conseqüência uma caracterização das álgebras de Koszul com dimensão global três, que satisfaçam a propriedade pd $\frac{r}{r^{2}}=2$, através da condição de $r^{2}$ ser um módulo de Koszul, de dimensão projetiva menor ou igual à 2 , (cf. Teorema 2.9).

Para descrever as álgebras de Koszul inclinadas, introduzimos o conceito de aplicações poço de torção,(cf. Proposição 4.11), que nos permitiu identificar quais são os somandos diretos do $\Lambda$-módulo inclinante $T$, que definem a cobertura projetiva do radical do $\Gamma$-módulo projetivo $P_{l}=\operatorname{Hom}_{\Lambda}\left(T, T_{l}\right)$, para $T_{l}$ um $\Lambda$-módulo indecomponível, com $T_{l} \in$ add $T$. Apesar de nosso resultado ter um caráter geral, no sentido de ser válido sobre $k$-álgebras de 
dimensão finita, não necessariamente hereditárias, ele se mostrou de mais fácil aplicação, no caso de álgebras inclinadas, por causa dos resultados conhecidos para as aljavas de Auslander-Reiten, $\Gamma_{\Lambda}$.

Para obtermos estes resultados, trabalhamos primeiramente com a classe de álgebras Brenner-Butler inclinadas ou simplesmente, as álgebras BBinclinadas, $\operatorname{End}_{\Lambda}(T)$, para $T=\tau^{-} S_{i} \oplus \bigoplus_{\substack{j \neq i \\ j=1}}^{n} P_{j}$, onde $\tau^{-}$é o funtor $\operatorname{Tr} D$, e os $P_{j}^{\prime} s$ são projetivos indecomponíveis tais que $\Lambda=\bigoplus_{j=1}^{n} P_{j} \quad$ e $\quad \tau^{-} S_{i} \neq 0$.

Mostramos que $\Gamma=\operatorname{End}_{\Lambda}(T)$ é uma álgebra de Koszul, descrevemos os $\Gamma$-módulos simples, assim como a aljava ordinária associada à $\Gamma$ e a classe dos módulos de Koszul sobre $\Gamma$, que, em muitas situações, é infinita, (cf cap. 3). Esta parte do trabalho, além de nos fornecer um exemplo detalhado para o caso das $B B$-inclinadas, nos deu indicações importantes, para o caso das álgebras inclinadas em geral, além de aumentar nosso interesse nesta classe de álgebras.

Como consequência da caracterização que obtivemos das álgebras Koszul inclinadas, apresentamos uma nova demonstração para o fato de que as álgebras inclinadas generalizadas do tipo $A_{n}$ (cf. exemplo 4.9.4, no cap. 4) são monomiais quadráticas, usando um lema apresentado em [AS,5], e métodos diagramáticos simples, que nos permitiu, inclusive, descrever a aljava destas álgebras.

Na direção de entender quais são os módulos de Koszul sobre as álgebras inclinadas surgiram respostas à outras questões, como as que foram apresentadas no trabalho em [GMRSZ]. Seja $\Gamma$ uma $k$-álgebra graduada e $\mathcal{L}(\Gamma)$ a classe dos $\Gamma$-módulos graduados com apresentação linear. Seja $\mathcal{K}(\Gamma)$ a classe dos $\Gamma$-módulos de Koszul. Em [GMRSZ], os autores colocam o problema de 
decidir para que tipo de álgebras estas classes seriam coincidentes e apresentam uma resposta afirmativa, para o caso das álgebras hereditárias e das monomiais de dimensão global finita.

Neste trabalho, mostramos que esta resposta é também afirmativa para as BB-inclinadas (cf. seção 3.5), mas que pode não valer para as inclinadas em geral, assim como para as $k$-álgebras $\mathbb{Z}$-graduadas de dimensão global 2 , (cf. Prop. 2.12).

Nosso trabalho ficou dividido da maneira que expomos a seguir.

No capítulo 1, apresentamos parte da teoria básica necessária para a compreensão de nosso texto.

No capítulo 2, apresentamos uma caracterização das álgebras quadráticas graduadas, e uma caracterização parcial das álgebras de dimensão global 3, através do radical quadrado da álgebra. Também neste capítulo, damos uma descrição dos módulos de Koszul sobre álgebras de dimensão global 2, respondendo, para uma situação mais geral, nossa terceira pergunta inicial neste trabalho, (cf. Proposição 2.12).

No capítulo 3, nosso principal resultado foi mostrar que as álgebras BBinclinadas são álgebras de Koszul, (cf. Teorema 3.14), para alcançar este resultado, fizemos um estudo detalhado desta classe de álgebras. Na seção 3.5, apresentamos um estudo detalhado da classe dos módulos de Koszul sobre as álgebras BB-inclinadas. Também, apresentamos um caso de álgebras inclinadas, que generaliza as BB-inclinadas, e que são álgebras de Koszul, quando o ideal da apresentação é um ideal graduado,(cf. Teorema 3.25).

No capítulo 4, além do estudo de algumas das possíveis graduações que podemos introduzir sobre anéis de endomorfismos, e do estudo detalhado da 
resolução projetiva de $\Gamma / r$, apresentamos nosso resultado central que caracteriza as álgebras de Koszul-inclinadas graduadas, (cf. Teorema 4.19), onde $\Gamma \cong k Q / I$, para $I$ um ideal graduado. Uma resposta parcial à questão da descrição da aljava de $\Gamma$, foi apresentada, como consequência destes resultados. Também, a partir deles, obtivemos uma nova demonstração para o fato de que as álgebras de tipo $A_{n}$ são quadráticas monomiais. Ainda neste capítulo, incluirnos alguns exemplos que procuram ilustrar nosso resultado central.

Como decorrência deste trabalho, podemos formular outras questões que, além de dar continuidade ao mesmo, abrem novos pontos de interesse no estudo da teoria de álgebras de Koszul. Por exemplo, sabendo que pode ocorrer que a classe dos módulos de Koszul sobre as álgebras Koszul-inclinadas pode ser infinita, obter uma caracterização destas algebras; ou, então, decidir quais são os morfismos entre somandos diretos do módulo inclinante que determinam um conjunto gerador de $r \Gamma / r^{2} \Gamma$, destas álgebras, ou então, decidir se é possível caracterizar as álgebras inclinadas, que tenham o ideal da apresentação graduado, através destes morfismos. 


\section{Capítulo 1}

\section{Preliminares}

Neste capítulo, apresentaremos a teoria básica sobre as álgebras inclinadas, as álgebras de Koszul e as representações de módulos com enfoque no que chamamos de teoria de Auslander-Reiten. Também, estaremos fixando a notação usada ao longo deste trabalho.

\subsection{Aljavas e Representações}

Nosso objetivo, neste parágrafo será introduzir as definições e conceitos básicos sobre aljavas e representações de álgebras através destas. Nossa principal referência foi o livro de Auslander-Reiten-Smalo, em [ARS].

Uma aljava $Q=\left(Q_{0}, Q_{1}\right)$ é um grafo orientado, onde $Q_{0}$ é um conjunto de vértices e $Q_{1}$ é um conjunto de flechas entre os vértices, sobre os quais são definidas aplicações $s: Q_{1} \longrightarrow Q_{0}$ e $e: Q_{1} \longrightarrow Q_{0}$ onde $s(\alpha)=i$ e $e(\alpha)=j$, quando $\alpha: i \longrightarrow j$ é uma flecha do vértice $i$ para o vértice $j$. Também denotamos por $\alpha_{i}^{j}$, ou $i \stackrel{\alpha}{\longrightarrow} j$, a flecha do vértice $i$ para o vértice $j$. Chamamos de caminho em $Q$, tanto a seqüência ordenada de flechas $\gamma=\alpha_{n}, \cdots \alpha_{1}$, onde $e\left(\alpha_{j}\right)=s\left(\alpha_{j+1}\right)$ para $1 \leq j<n$, como o símbolo $e_{i}$, para $i \in Q_{0}$. O caminho $e_{i}$ é dito trivial e definimos $s\left(e_{i}\right)=e\left(e_{i}\right)=i$. Dizemos que $\gamma$, um caminho não-trivial, é um ciclo orientado quando $s\left(\alpha_{1}\right)=e\left(\alpha_{n}\right)$.

No caso em que a aljava é finita, ou seja, em que $Q_{0}$ e $Q_{1}$ são conjuntos finitos, definimos uma álgebra de caminhos $k Q$ sobre um corpo $k$ como sendo o $k$-espaço vetorial gerado pelos caminhos em $Q$, cujo produto é definido da 
maneira a seguir. Sejam $\gamma=\alpha_{n} . \cdots, \alpha_{1}$ e $\eta=\beta_{m} . \cdots \beta_{1}$ caminhos em $k Q$. Definimos o produto de $\gamma$ por $\eta$ por:

$$
\gamma \cdot \eta=\left\{\begin{array}{ccc}
\alpha_{n} \cdot \cdots \cdot \alpha_{1} \cdot \beta_{m} \cdot \cdots \cdot \beta_{1} & \text { se } & s\left(\alpha_{1}\right)=e\left(\beta_{m}\right) \\
0 & \text { caso contrário }
\end{array}\right.
$$

Se $Q$ é uma aljava finita, é fato conhecido que $k Q$ é uma $k$-álgebra de dimensão finita se e somente se $Q$ não contém ciclos orientados. Temos que $k Q$ é uma álgebra hereditária.

Se $I$ é um ideal de $k Q$ tal que $L^{n} \subset I \subset L^{2}$, onde $L$ é o ideal de $k Q$ gerado pelas flechas e $n \geq 2$, dizemos que $I$ é um ideal admissível. O resultado a seguir , devido a Gabriel, nos diz como podemos relacionar as álgebras de dimensão finita e as álgebras de caminhos,(cf. [ARS], no capítulo III).

Teorema : (Gabriel) Seja $\Lambda$ uma álgebra de dimensão finita sobre um corpo $k$ algébricamente fechado, básica e indecomponivel. Então, $\Lambda$ é isomorfa ao quociente de uma álgebra de caminhos $k Q$ por um ideal admissível $I$, onde $Q$ é uma aljava finita e conexa.

A demonstração deste resultado tem como base as idéias que apresentamos em seguida e podem ser encontradas em [ARS, em III.1.9]. Consideremos $E=\left\{e_{1}, \cdots, e_{n}\right\}$ o conjunto formado pelo sistema completo de idempotentes ortogonais primitivos de $\Lambda$ e $R=\left\{r_{1}, \cdots, r_{t}\right\}$ um conjunto dos elementos em $r$, o radical de $\Lambda$, tal que as imagens $\bar{r}_{1}, \cdots, \bar{r}_{t}$ em $r / r^{2}$ geram $r / r^{2}$ como $\Lambda / r$-módulo. Então $E \cup R$ gera $\Lambda$ como $k$-álgebra.

A partir dos conjuntos $E$ e $R$ definidos acima, podemos construir a aljava $Q_{\Lambda}$ de $\Lambda$, como segue. Consideremos $Q_{0}$ o conjunto de vértices cuja cardinalidade é igual a do conjunto $E$ acima, e o denotaremos por $\{1, \cdots, n\}$. Fixemos uma base para o $k$-espaço vetorial $e_{j} \frac{r}{r^{2}} e_{i}$, cujos elementos denotaremos por $r_{s}(i, j)$, onde $s=1, \cdots, m$, para $m=\operatorname{dim}_{k}\left(e_{j} \frac{r}{r^{2}} e_{i}\right)$. Consideremos $Q_{1} \circ$ conjunto das flechas da aljava de $\Lambda$, cujos elementos denotaremos por $\alpha_{s}(i, j)$, com $i, j \in Q_{0}$, onde $s=1, \cdots, m$. A aljava $Q$ está definida por estes conjuntos $Q_{0}$ e $Q_{1}$ dados acima, e pelas funções $s\left(\alpha_{s}(i, j)\right)=i, e\left(\alpha_{s}(i, j)\right)=j$. A aljava $Q$ é chamada de aljava ordinária associada à $\Lambda$, ou simplesmente a aljava de $\Lambda$.

Consideremos, agora, o $k$-espaço vetorial $k Q$. Podemos definir um morfismo de álgebras $\varphi: k Q \longrightarrow \Lambda$, tomando $\phi(i)=e_{i}$, e $\phi\left(\alpha_{s}(i, j)\right)=r_{s}(i, j)$, como acima, e estendemos por linearidade. Pode-se provar que nuc $\varphi$ satisfaz a propriedade de $L^{n} \subset$ nuc $\varphi \subset L^{2}$, com $n \geq 2$, onde $L$ é o ideal de $k Q$ gerado pelas flechas, e assim, concluir que nuc $\varphi$ é um ideal admissível. 
Ao tomarmos $I=$ nuc $\varphi$, teremos que $\Lambda \cong k Q / I$. A aljava $Q$ será denotada por $Q(\Lambda)$, ou $Q_{\Lambda}$, sempre que for necessário diferenciá-la da aljava de outras álgebras. O ideal $I$ é chamado de ideal da apresentação de $\Lambda$ sobre $k Q_{\Lambda}$.

Cada $\Lambda$-módulo dado por $\Lambda e_{j} \operatorname{com} j=1, \ldots, n$ é um módulo projetivo indecomponível tal que, se existe um morfismo não-nulo $\varphi: \Lambda e_{j} \longrightarrow \Lambda e_{s}$, com $j \neq s$, então existe um caminho em $Q(\Lambda)$, fora de $I$, do vértice $s$ para o vértice $j$. Dado um caminho $\gamma=\alpha_{n}, \cdots, \alpha_{1}$ em $Q(\Lambda)$ de $s$ para $j$ podemos definir um morfismo $\varphi_{\gamma}: \Lambda e_{j} \longrightarrow \Lambda e_{s}$ dado por $\varphi_{\gamma}\left(e_{j}\right)=\gamma e_{j}$, que será não-nulo, quando $\gamma$ não pertencer a $I$.

As álgebras $\Lambda=k Q / I$ poderão ser apresentadas, neste trabalho, através do grafo de sua aljava ordinária e relações que contenha. As relações de comutatividade, quando existirem, serão indicadas por pontilhados, segundo a conveniência do texto, como na figura abaixo:

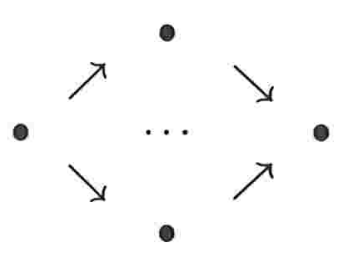

Vejamos alguns exemplos:

(1) Seja $\Lambda=k[x] /\left\langle x^{2}\right\rangle$. Então, $\Lambda$ tem a apresentação dada pela aljava $1 \stackrel{\alpha}{\longrightarrow} 1$ (um laço) e a relação $\alpha^{2}=0$.

(2) Seja $\Lambda$ a álgebra de matrizes triangulares superiores, $(3 \times 3)$, sobre um corpo $k$, algebricamente fechado. Então, $\Lambda=k Q$, onde $Q$ é a aljava

$$
1 \stackrel{\alpha}{\longrightarrow} 2 \stackrel{\beta}{\longrightarrow} 3 \text {. }
$$

Observamos que $\Lambda$ não contém relações, isto é, $I=(0)$. Este é um exemplo de álgebra hereditária.

É fato conhecido que a categoria $\Lambda$-mod e a categoria das $k$-representações da aljava de $\Lambda$ com relações, são categorias equivalentes. Denotemos por $\operatorname{Rep}(Q, I)$, a categoria das $k$-representações de uma álgebra dada por uma aljava e relações, que é definida da seguinte maneira : 
(i) Os objetos são dados pelas uplas $\left(\left(M_{j}\right)_{j \in Q_{0}},\left(f_{\alpha}\right)_{\alpha \in Q_{1}}\right)$ tal que $M_{j}$ é um $k$-espaço vetorial para cada j, e $f_{\alpha}: M_{s(\alpha)} \longrightarrow M_{e(\alpha)}$ é uma $k$-transformação linear.

(ii)Os morfismos entre os objetos $\left(\left(M_{j}\right)_{j},\left(f_{\alpha}\right)_{\alpha}\right)$ e $\left.\left(\left(N_{j}\right)_{j},\left(g_{\alpha}\right)_{\alpha}\right)\right)$ são dados por uplas $F=\left(F_{j}\right)$, onde $F_{j}: M_{j} \longrightarrow N_{j}$ é uma transformação linear tal que o diagrama abaixo é comutativo :

$$
\begin{array}{lll}
M_{j} \stackrel{f_{\alpha}}{\longrightarrow} & M_{s} \\
F_{j} \downarrow & & \downarrow F_{s} \\
N_{j} & \stackrel{g_{\alpha}}{\longrightarrow} & N_{s}
\end{array}
$$

Dados um caminho $\gamma=\alpha_{m} . \cdots \alpha_{1}$ em $Q$, e uma $k$-representação $M=$ $\left.\left(\left(M_{j}\right)_{j},\left(f_{\alpha}\right)_{\alpha}\right)\right)$, podemos definir uma $k$-transformação linear $M(\gamma): M_{s\left(\alpha_{1}\right)} \rightarrow$ $M_{e\left(\alpha_{m}\right)}$, por $M(\gamma)=f_{\alpha_{m}} \circ \cdots \circ f_{\alpha_{1}}$, estendendo-a por linearidade, quando se tratar de combinações lineares de caminhos em $k Q$. Assim, a upla $\left(\left(M_{j}\right),\left(f_{\alpha}\right)\right)$ com as propriedades acima é uma $k$-representação em $\operatorname{Rep}(Q, I)$, se dado $\rho=\sum_{\gamma} \lambda_{\gamma} \gamma \in I, \operatorname{com} \alpha_{\gamma} \in k$ e $\gamma$ caminhos em $k Q$, então $M(\rho)=0$.

\subsection{Teoria de Auslander-Reiten}

Vamos neste parágrafo, fixar a notação e apresentar mais alguns conceitos básicos da teoria de representações de álgebras, que utilizaremos ao longo deste trabalho. Mais detalhes e resultados podem ser encontrado em nossas referências, como por exemplo, em [ARS].

Seja $\Lambda$ uma $k$-álgebra de dimensão finita.

Denotaremos por $\Lambda$-mod a categoria dos módulos finitamente gerados sobre $\Lambda$. A menos de menção em contrário, os módulos sobre $\Lambda$ são módulos à esquerda. Denotaremos por ind- $\Lambda$ a subcategoria plena de $\Lambda$-mod cujos objetos são as classes de isomorfia dos módulos indecomponíveis. Sempre que não ocorram confusões, estaremos identificando um módulo indecomponível com sua classe de isomorfia. Denotaremos por add $M$, a subcategoria plena de $\Gamma$-mod, cujos objetos são os módulos que são somas diretas de somandos diretos de $M$. 
Dados $A$ e $B$ em $\Lambda$-mod, denotaremos o módulo $\operatorname{Hom}_{\Lambda}(A, B)$, abreviadamente por $(A, B)$, para simplificar nossa notação, quando for necessário.

Denotaremos o radical de uma álgebra $\Lambda$ por $r$, quando não houver possibilidade de confusões. Caso contrário, escrevemos $r=r_{\Lambda}$, para diferenciar do radical de outras álgebras. Dado um $\Lambda$-módulo $M$, denotaremos a cobertura projetiva de $M$ por $P_{\Lambda}(M)$ ou $P_{(0)}(M)$, ou ainda, $P_{(0)}$.

Quando não for especificado, o $\Lambda$-módulo $P_{j}$ é o projetivo indecomponível onde o topo $\frac{P_{j}}{r P_{j}}$, é dado pelo $\Lambda$-módulo simples $S_{j}$, e $I_{j}$ é o injetivo indecomponível cujo socle é $S_{j}$, associados ao vértice $j$.

Sobre álgebras de Artin podem ser definidos o que chamamos de morfismo quase cindido. Trata-se de um importante conceito da teoria de representações de álgebras, devido a M. Auslander e I. Reiten, que fundamentou os alicerces dos novos caminhos que a área tomou, a partir de então. Vejamos do que se trata.

Definição 1.1 Sejam $B$ e $C$ módulos sobre uma álgebra de Artin e seja $f: B \longrightarrow C$ um morfismo não-nulo. Dizemos que $f$ é um morfismo quase cindido à direita, se satisfaz as seguintes propriedades:

(i) $f$ não é um epimorfismo que cinde.

(ii) todo morfismo $X \longrightarrow C$ que não seja epimorfismo que cinde se fatora através de $f$.

Um morfismo quase cindido à direita, não-nulo, $f: B \rightarrow C$ é dito minimal, se todo morfismo $g: B \rightarrow B$ tal que $f=f \circ g$, é um isomorfismo.

Um exemplo de morfismo quase cindido à direita, minimal, é dado pela inclusão $r P \hookrightarrow P$, onde $P$ é um projetivo indecomponível. Na literatura, pode-se encontrar este morfismo denominado por morfismo poço ou simplesmente poço.

$\mathrm{O}$ conceito de morfismo quase cindido à esquerda pode ser definido dualmente.

Definição 1.2 Uma seqüência exata curta de módulos sobre uma álgebra de Artin, como abaixo:

$$
0 \longrightarrow n u c f \longrightarrow B \stackrel{f}{\longrightarrow} C \longrightarrow 0
$$

tal que $f$ é um morfismo quase cindido à direita minimal é chamada de sequência quase cindida. 
Estas seqüências estão unicamente determinadas, a menos de isomorfismos, pelo módulo $C$ (ou dualmente, por $A=n u c(f)$ ). Observamos que, é possível provar que $C$ é um módulo indecomponível, mas que $B$ nem sempre o será.

As funções componentes de $f$ são os morfismos irredutiveis, (cf na pag. 166, V.5 em [ARS]).

No caso de álgebras de Artin, podemos definir uma aljava, associada à categoria dos $\Lambda$-módulos finitamente gerados, onde os vértices são dados pelas classes de isomorfia dos módulos indecomponíveis e as flechas por morfismos irredutíveis entre estes módulos. Denotaremos por $\Gamma_{A}$ esta aljava, onde $A$ é a álgebra de Artin em questão, e a chamaremos, como se faz usualmente, de aljava de Auslander-Reiten de $A$, ou simplesmente a aljava de $A R$ de $A$. Por exemplo, se considerarmos a álgebra $\Lambda$ definida pelo exemplo 2, acima, teremos que aljava de $A R$ de $\Lambda$ é dada por:

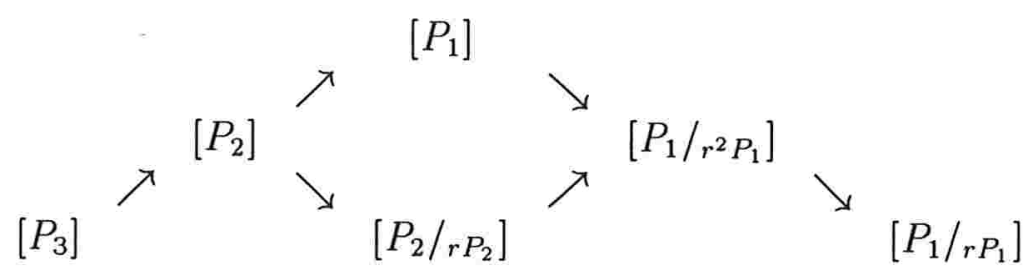

onde [.] é a classe de isomorfia dos módulos indecomponíveis sobre $\Lambda, P_{i}$ é o $\Lambda$-módulo projetivo indecomponível associado ao vértice $i=1,2,3$, e as flechas são morfismos irredutíveis entre os módulos indecomponíveis.

As álgebras hereditárias dentro da teoria de representações são as álgebras cuja estrutura e categorias de módulos são bem conhecidos. Sobre estas, existem duas classes interessantes de módulos, a dos preprojetivos e a dos preinjetivos. Tais classes fornecem uma descrição de certas componentes da aljava de $A R$ da álgebra hereditária, que contém os módulos projetivos e injetivos, respectivamente, na forma descrita abaixo.

Sobre uma algebra hereditária $\Lambda=k Q$, um $\Lambda$-módulo $M$ é dito preprojetivo (respec. preinjetivo), quando existe um inteiro não-negativo tal que $(D T r)^{n} M$ (respec. $\left.(\operatorname{Tr} D)^{n} M\right)$, é um módulo projetivo (respec. injetivo), não nulo, onde $D$ e $\operatorname{Tr}$ são funtores dados por

$$
\begin{gathered}
D=H_{\Lambda}(-, k): \Lambda-\bmod \longrightarrow \Lambda^{o p}-\bmod \\
\text { e } \operatorname{Tr}(-) \cong \operatorname{Ext}_{\Lambda}^{1}(-, \Lambda) .
\end{gathered}
$$


O módulo $(D T r)^{n} M$, é chamado de $n$-ésimo transladado de $M$ e denotado por $\tau^{n} M$. Analogamente, $(\operatorname{Tr} D)^{n} M$, também denotado por $\tau^{-n} M$, é chamado de m-ésimo transladado inverso de $\mathrm{M}$.

Pelo Lema 1.8, pag.263 em [ARS], vimos que dada $\Lambda$, uma álgebra de Artin, hereditária, tal que uma componente $\mathcal{C}$ da aljava de $\mathrm{AR}$ de $\Lambda$, contém um vértice preprojetivo, então todos os vértices desta componente são preprojetivos. Tais componentes são chamadas de componente preprojetiva da aljava de AR de $\Lambda$. Pode-se provar que, uma componente da aljava de AR de álgebras $\Lambda$, como acima, é uma componente preprojetiva (preinjetiva) se esta componente contém todos os módulos projetivos (respec. injetivos).

Também como conseqüência deste lema, segue que na aljava de AR de uma álgebra hereditária, todo módulo preprojetivo (preinjetivo) está ligado a um módulo projetivo (respec. injetivo), por uma cadeia de morfismos irredutiveis.

\section{3 Álgebras inclinadas}

Neste parágrafo, vamos definir as álgebras inclinadas e apresentar resultados e propriedades importantes sobre estas álgebras, e que utilizaremos neste trabalho. Os resultados, que aqui expomos, podem ser encontrados em $[\mathrm{AS}, 1]$ e $[\mathrm{HR}]$.

Definição 1.3 Sejam $\Lambda$ uma k-álgebra de dimensão finita sobre um corpo $k$ e $T$ um $\Lambda$-módulo. Dizemos que $T$ é um módulo inclinante se satisfaz as seguintes propriedades:

(i) $p d_{\Lambda} T \leq 1$

(ii) $\operatorname{Ext}_{\Lambda}^{1}(T, T)=0$

(iii) existe uma sequência exata curta $0 \longrightarrow \Lambda \longrightarrow T^{\prime} \longrightarrow T^{\prime \prime} \longrightarrow 0$, com $T^{\prime}$ e $T^{\prime \prime}$ em addT.

Dizemos que o $\Lambda$-módulo $T$ é livre de multiplicidade se os somandos diretos indecomponíveis de $T$, são dois a dois, não isomorfos.

Seja $\Gamma=\operatorname{End}_{\Lambda}(T)^{o p}$, o anel de endomorfismo de $T$ sobre $\Lambda$. É fácil verificar que o $\Gamma$-módulo dado por $\operatorname{Hom}_{\Lambda}\left(T, T^{\prime}\right)$, onde $T^{\prime} \in$ add $T$, é um 
módulo projetivo. Também, é fato conhecido que, o posto de Grothendieck de $\Lambda$ e $\Gamma$ coincidem.

Se $\Lambda$ é uma álgebra hereditária, o anel de endomorfismo $\Gamma=\operatorname{End}_{\Lambda}(T)^{o p}$ é chamado de álgebra inclinada (cf. [HR]). Sabemos por [AS,1], que, neste caso, gldim $\Gamma \leq 2$. Neste mesmo trabalho, foi mostrado que um $\Lambda$-módulo $T$ é um módulo inclinante se e sómente se satisfaz os itens (i) e (ii) da definição acima e a seguinte afirmação: "o número de classes de isomorfia de somandos diretos indecomponíveis de $T$ é igual ao número de classes de isomorfia de $\Lambda$-módulos simples, ou seja, é igual ao posto do grupo de Grothendieck de $\Lambda "$.

A partir de um $\Lambda$-módulo inclinante $T$, podemos definir duas classes de módulos em $\Lambda$-mod, a categoria dos módulos finitamente gerados em $\Lambda$, como se segue:

$$
\mathcal{T}(T)=\left\{M \in \Lambda-\bmod : \operatorname{Ext}_{\Lambda}^{1}(T, M)=0\right\}
$$

$\mathrm{e}$

$$
\mathcal{F}(T)=\left\{N \in \Lambda-\bmod : \operatorname{Hom}_{\Lambda}(T, M)=0\right\}
$$

Prova-se que $\mathcal{T}(T)$ é a subcategoria plena dos $\Lambda$-módulos gerados por $T$ e que $\mathcal{F}(T)$ é a subcategoria plena dos $\Lambda$-módulos cogerados por $T$. A classe $\mathcal{T}=\mathcal{T}(T)$ é chamada de classe dos módulos de torção gerados por $T$ ou simplesmente módulos de torção e $\mathcal{F}=\mathcal{F}(T)$ a classe dos módulos livre de torção cogerados por $T$ ou módulos livre de torção. Nesta situação que apresentamos, temos que o par $(\mathcal{T}(T), \mathcal{F}(T))$ forma uma teoria de torção para a categoria de $\Lambda$-módulos. Um resultado devido a Brenner-Butler (cf. $[\mathrm{BB}]$ e $[\mathrm{AS} 1]$ ) relaciona as subcategorias acima com as seguintes subcategorias plenas de $\Gamma=\operatorname{End}_{\Lambda}(T)^{o p}$ definidas por:

$$
\begin{gathered}
\mathcal{X}=\mathcal{X}(T)=\{M \in \Gamma-\bmod : M \underset{\Gamma}{\bigotimes} T=0\} \\
\mathcal{Y}=\mathcal{Y}(T)=\left\{N \in \Gamma-\bmod : \operatorname{Tor}_{1}^{\Gamma}(N, T)=0\right\}
\end{gathered}
$$

O resultado abaixo é uma versão do Teorema 2.1, pag. 20, apresentado no trabalho de I.Assem, em [AS,1]. Aqui, estaremos considerando módulos à esquerda. Vejamos como.

Teorema 1.4 (cf. em [AS,1], Teorema 2.1, pag. 20): Sejam $\Lambda$ umakálgebra de dimensão finita, $T$ um $\Lambda$-módulo à esquerda inclinante, e $\Gamma=$ 
$\operatorname{End}_{\Lambda}(T)^{o p}$. Então, o $\Gamma$-módulo à direita $T$ é um módulo inclinante e $\Lambda=$ $\operatorname{End}_{\Gamma}\left(T_{\Gamma}\right)$. Mais ainda, as subcategorias $\mathcal{T}(T)$ e $\mathcal{Y}(T)$ de módulos à esquerda, são equivalentes sob a restrição dos funtores $\operatorname{Hom}_{\Lambda}(T,-) e-\bigotimes_{\Gamma} T$, os quais são mutuamente inversos; e similarmente, as subcategorias $\mathcal{F}(T)$ e $\mathcal{X}(T)$ são equivalentes sob a restrição dos funtores $\operatorname{Ext}_{\Lambda}^{1}(T,-)$ e $\operatorname{Tor}_{\Lambda}^{1}(-, T)$ os quais são, novamente, mutuamente inversos.

As seguintes propriedades homológicas para os $\Gamma$-módulos são válidas:

(i) $p d_{\Gamma}\left[\operatorname{Hom}_{\Lambda}(T, X)\right] \leq \operatorname{pd}_{\Lambda} X$, para todo $X \in \mathcal{T}(T)$

(ii) $p d_{\Gamma}\left[\operatorname{Ext}_{\Lambda}^{1}(T, Y)\right] \leq 1+\operatorname{pd}_{\Lambda}(Y)$, para todo $Y \in \mathcal{F}(T)$

No caso em que $\Lambda$ é hereditária, vamos ter que $\operatorname{pd}_{\Gamma} X \leq 1$, para cada

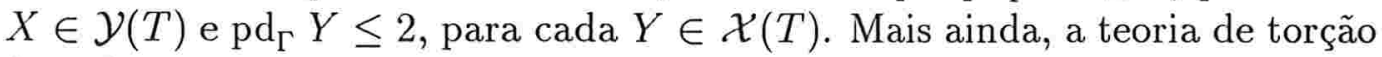
$(\mathcal{X}, \mathcal{Y})$ cinde a categoria de módulos finitamente gerados sobre $\Gamma$, ou seja, todo módulo indecomponível sobre $\Gamma$ ou pertence a $\mathcal{X}$ ou pertence a $\mathcal{Y}$.

\section{4 Álgebras de Koszul}

Vamos, agora, apresentar as definições e resultados importantes para nosso trabalho, sobre a teoria das álgebras de Koszul. Todos os resultados aqui expostos, podem ser encontrados nos trabalhos de Green e MartinezVilla, em [GM, 1 e 2].

Definição 1.5 Uma $k$-álgebra $\mathbb{Z}$-graduada $\Gamma$ é uma familia $\left\{\Gamma^{(i)}, \phi_{i j}\right\}_{i, j \in \mathbb{Z}}$ com $\Gamma^{(i)} k$-espaço vetorial e $\phi_{i j}: \Gamma^{(i)} \underset{\Gamma^{(0)}}{\bigotimes} \Gamma^{(j)} \longrightarrow \Gamma^{(i+j)}$, uma $k$-transformação linear tal que:

(i) $\Gamma^{(0)}$ é uma k-álgebra

(ii) $\Gamma^{(i)}$ é um $\Gamma^{(0)}-\Gamma^{(0)}-$ bimódulo 
(iii) o diagrama abaixo é comutativo:

$$
\begin{array}{ccc}
\Gamma^{(i)} \underset{\Gamma^{(0)}}{\otimes} \Gamma_{\Gamma^{(j)}}^{\otimes} \Gamma^{(k)} & \stackrel{\phi_{i j} \otimes 1}{\longrightarrow} & \Gamma^{(i+j)} \underset{\Gamma^{(0)}}{\otimes} \Gamma^{(k)} \\
\downarrow 1 \otimes \phi_{j k} & & \downarrow \phi_{(i+j) k} \\
\Gamma^{(i)} \underset{\Gamma^{(0)}}{\otimes} \Gamma^{(j+k)} & \underset{\phi_{i(j+k)}}{\longrightarrow} & \Gamma^{(i+j+k)}
\end{array}
$$

para cada $i, j, k$.

Denotaremos $\Gamma=\bigsqcup_{i} \Gamma^{(i)}$. ocorre que:

Dizemos que $\Gamma=\underset{i \in \mathbb{Z}}{\amalg} \Gamma^{(i)}$ é 1-gerada e de decomposição básica, quando

(1) $\phi_{i j}$ é sobrejetora para $i, j$.

(2) $\Gamma^{(0)}$ é isomorfa a um produto finito de cópias de $k$.

(3) cada componente homogênea $\Gamma^{(i)}$ é um $\Gamma^{(0)}-\Gamma^{(0)}$-bimódulo de comprimento finito.

Uma $k$-álgebra é $\mathbb{N}$-graduada quando for uma álgebra $\mathbb{Z}$-graduada, cujas componentes de grau negativo são todas nulas. Neste trabalho, estaremos considerando álgebras de dimensão finita sobre $k, \mathbb{N}$-graduadas, 1-gerada e de decomposição básica.

Claramente, as álgebras de caminhos $k Q$, onde $Q$ é uma aljava finita, são $k$-álgebras $\mathbb{N}$-graduadas, 1 -geradas e de composição básica. Dizemos que um ideal $I$ de $k Q$, é um ideal graduado se $(k Q)_{s} I_{n} \subset I_{(n+s)}$. Se $\Gamma$ é o quociente de uma álgebra de caminhos por um ideal admissível graduado, então $\Gamma$ é, também, uma $k$-álgebra $\mathbb{N}$-graduada, 1-gerada e de decomposição básica.

Por outro lado, se $\Gamma$ for uma $k$-álgebra graduada, 1-gerada e de decomposição básica teremos que $r=\coprod_{i \geq 1} \Gamma^{(i)}$, é o radical de Jacobson graduado de $\Gamma$. Mais ainda, temos que $\Gamma$ é o quociente de uma álgebra de caminhos $k Q$ por um ideal admissível graduado $I$, (cf prop.2.3 em [GM,1]).

Dizemos que um $\Gamma$-módulo graduado $M=\coprod_{i \geq j} M^{(i)}$ é gerado em grau $j$, se $M \neq 0$ e $\Gamma^{(k)} \cdot M^{(j)}=M^{(j+k)}$, para cada $k$. Se $s$ é um inteiro, definimos 
o $s$-translado de $M$, que denotaremos por $M[s]$, como sendo o $\Gamma$-módulo graduado dado por $\coprod_{i} N^{(i)}$, onde $N^{(i)}=M^{(i-s)}$, com a estrutura de módulo dada por $M$.

A categoria dos $\Gamma$-módulos graduados finitamente gerados é a categoria cujos objetos são os módulos graduados e cujos morfismos $f: \oplus_{j} M^{(j)} \longrightarrow$ $\oplus_{j} N^{(j)}$ são tais que $f\left(M^{(j)}\right) \subset N^{(j)}$. Denotaremos esta categoria por gr $\Gamma$. Denotaremos por $\operatorname{gr}_{(0)} \Gamma$ a subcategoria plena de gr $\Gamma$, dos módulos gerados em grau zero.

Definição 1.6 Um $\Gamma$-módulo $M$, gerado em grau zero, é dito um módulo de Koszul quando $M$ possui uma resolução linear, a saber, se existe uma $\Gamma$-resolução projetiva de $M$ em $\mathrm{gr} \Gamma$ :

$$
\cdots \longrightarrow P_{(3)}(M) \stackrel{f_{3}}{\longrightarrow} P_{(2)}(M) \stackrel{f_{2}}{\longrightarrow} P_{(1)}(M) \stackrel{f_{1}}{\longrightarrow} P_{(0)}(M) \stackrel{f_{0}}{\longrightarrow} M \longrightarrow 0
$$

tal que $P_{(j)}(M)$ é gerado em grau $j$ para cada $j \geq 0$.

Dizemos que um $\Gamma$-módulo $M$, tem uma apresentação linear quando $P_{(0)}(M)$ e $P_{(1)}(M)$ são gerados em graus zero e um, respectivamente; e que $M$ tem resolução linear de comprimento $s$ quando $P_{(j)}(M)$ é gerado em grau $j$, para $0 \leq j \leq s$.

Definição 1.7 Uma k-álgebra graduada $\Gamma$, 1-gerada e de decomposição básica, é uma álgebra de Koszul quando todo Г-módulo simples é um módulo de Koszul.

Por exemplo, as álgebras hereditárias são álgebras de Koszul, pois todo módulo simples tem resolução linear de comprimento um, e todo módulo sobre estas álgebras tem dimensão projetiva um.

São conhecidas propriedades interessantes sobre módulos de Koszul em álgebras de Koszul, algumas das quais exporemos a seguir. Seja $\Gamma$ uma $k$ álgebra graduada, 1-gerada e de decomposição básica. Suponha que $\Gamma$ seja uma álgebra de Koszul. É conhecido que:

(i)Se $M$ é um $\Gamma$-módulo de Koszul então $r M$ é um $\Gamma$-módulo de Koszul. Como todo projetivo é um módulo de Koszul segue que, sobre álgebras de Koszul, radicais de projetivos e potências destes, são módulos de Koszul.

(ii) Um $\Gamma$-módulo graduado $M$, gerado em grau zero, é Koszul se e somente se $\Omega^{i}(M)[-i]$ é um $\Gamma$-módulo com resolução linear para cada $i>0$, onde $\Omega^{i}(M)$ é o $i$-ésimo syzygy de $M$. 
Os resultados acima, podem ser encontrados em [GM,1].

Definição 1.8 Um ideal I de uma álgebra de caminhos $k Q$, é dito ideal quadrático, quando I é gerado por combinações lineares de caminhos de comprimento dois. A álgebra de dimensão finita sobre o corpo $k$, básica, $\Gamma$ é dita quadrática quando admite uma apresentação $\Gamma=\frac{k Q}{I}$, onde $I$ é um ideal quadrático.

Sabemos, por [GM,1], que uma álgebra graduada é quadrática, se e sómente se, $I$ satisfaz a propriedade $I \cap L^{3}=I L+L I$, onde $L$ é o ideal de $k Q(\Gamma)$ gerado pelas flechas da aljava $Q(\Gamma)$. Ainda em [GM,1], os autores mostraram que as álgebras de Koszul são quadráticas mas, a recíproca não vale em geral. Apresentamos abaixo, um exemplo devido a E.L.green, que ilustra este fato. No entanto, para álgebras de dimensão global dois, os autores mostram que $\Gamma$ é álgebra de Koszul se e somente se $\Gamma$ é álgebra quadrática.

Exemplo:(green) Seja $\Lambda$ a $k$-álgebra dada por aljavas e relações da seguinte maneira:

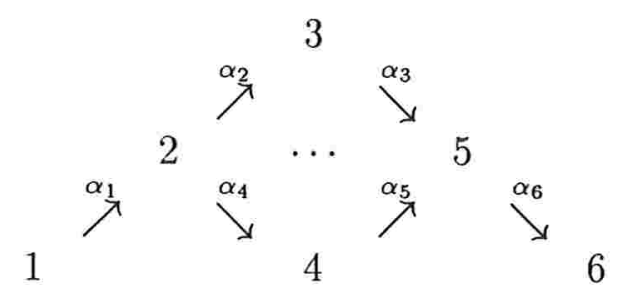

com as relações $\alpha_{2} \alpha_{1}=0=\alpha_{6} \alpha_{5}$ e $\alpha_{3} \alpha_{2}=\alpha_{5} \alpha_{4}$. Observamos que esta álgebra é uma álgebra quadrática que não é Koszul, pois é fácil ver que o $\Lambda$-módulo simples $S_{1}$, associado ao vértice 1 , não é um módulo de Koszul.

Sabemos por [GM,2], que a ext-álgebra de $\Gamma$ dada por

$$
E(\Gamma)=\coprod_{i \geq 0} \operatorname{Ext}_{\Gamma}^{i}(\Gamma / r, \Gamma / r)
$$

é uma álgebra de Koszul, se $\Gamma$ o for. No caso de álgebras hereditárias $\Lambda$, temos que $E(\Lambda) \cong \Lambda^{\mathrm{op}} /\left({ }_{\left({ }^{o p}\right)_{\Lambda}^{2}}\right.$, onde $r_{\Lambda}$ é o radical de $\Lambda$.

Consideraremos sobre $\Gamma$-mod, a classe $\mathcal{L}(\Gamma)$ dos $\Gamma$-módulos com apresentação linear, ou seja, os $\Gamma$-módulos graduados $M$, gerados em grau zero, 
e com apresentação projetiva dada por:

$$
P_{(1)} \longrightarrow P_{(0)} \longrightarrow M \longrightarrow 0
$$

tal que $P_{(i)}$ é gerado em grau $i$, para $i=0,1$.

Observamos que todo módulo simples sobre uma $k$-álgebra de dimensão finita, tem apresentação linear.

Denotaremos por $\mathcal{K}(\Gamma)$, a classe dos módulos de Koszul em $\operatorname{gr}_{(0)} \Gamma$. É fato conhecido que sobre as álgebras hereditárias e as álgebras quadráticas monomiais, as classes $\mathcal{K}(\Gamma)$ e $\mathcal{L}(\Gamma)$ coincidem, (cf. em [GMRSZ]). 


\section{Capítulo 2}

\section{Sobre Álgebras de Koszul com dimensão global finita}

Neste capítulo, estaremos apresentando uma nova caracterização para as álgebras quadráticas graduadas e uma caracterização parcial das álgebras de Koszul com dimensão global 3. Apresentaremos, também, resultados interessantes referentes aos módulos de Koszul sobre álgebras de dimensão global dois, que tem, para este trabalho, um particular interesse, já que as álgebras inclinadas são um caso particular destas álgebras.

\subsection{Introdução}

Nesta seção apresentaremos mais algumas definições básicas e alguns resultados sobre as álgebras de Koszul, bem como introduziremos a notação usada no capítulo. Todos estes resultados, assim como as definições, podem ser encontrados em [GG] e [G-M, 1 e 2].

Sejam $k$ um corpo e seja $\Gamma$ uma $k$-álgebra $\mathbb{N}$-graduada, como foi definida no capítulo anterior.

Lembremos que uma $k$-álgebra $\mathbb{N}$-graduada $\Gamma=\coprod_{i>0} \Gamma^{(i)}$ é 1 -gerada, se as aplicações $\phi_{i j}$ são sobrejetoras, $\forall i, j \geq 0$, (cf. def 1.5$)$. Neste caso, vale que $\Gamma^{(i)} \Gamma^{(j)}=\Gamma^{(i+j)}$, ou equivalentemente, $\Gamma^{(i)}=\prod_{n=1}^{i} \Gamma^{(1)}=\left[\Gamma^{(1)}\right]^{i}$. 
É fato conhecido que se uma $k$-álgebra graduada $\Gamma=\coprod_{i \geq 0} \Gamma^{(i)}$ é 1-gerada então $\Gamma$ é isomorfo, como anel graduado ao anel graduado associado ao ideal $r$, onde $r$ é o ideal de $\Gamma$ dado por $r=\coprod_{i \geq 1} \Gamma^{(i)}$, (cf em [GM,1], Prop.2.2). Caso $\Gamma$ seja de decomposição básica, dizemos que $r$ é o radical de Jacobson graduado de $\Gamma$. Pelo trabalho apresentado por E. Green e R. Gordon em [GG], vimos que $r$ é um $\Gamma$-módulo graduado, gerado em grau um.

Ao longo deste capítulo, estaremos considerando $\Gamma$ uma $k$-álgebra graduada, de decomposição básica, 1-gerada e $r$ seu radical de Jacobson graduado. Pelos resultados apresentados po Green e Martinez-Villa em [GM,1], sabemos que uma álgebra nestas condições é isomorfa ao quociente de uma álgebra de caminhos $k Q$ por um ideal $I$, onde $Q$ é uma aljava finita e $I$ um ideal graduado, admissível. Assim, ao longo deste capítulo, estaremos considerando $\Gamma=k Q / I$ onde $Q$ é uma aljava finita, e $I$ é um ideal admissível, graduado.

Fixaremos, também, a Gr $\Gamma$-resolução projetiva de $\Gamma / r$ da seguinte maneira:

$$
\cdots \longrightarrow P_{(2)} \longrightarrow P_{(1)} \longrightarrow P_{(0)} \longrightarrow \Gamma / r \longrightarrow 0
$$

onde $P_{(0)}=\Gamma$ e $r$ é o radical de Jacobson graduado de $\Gamma$.

Considere sobre $\Gamma$ um módulo graduado $M=\coprod_{i} M^{(i)}$. Lembremos que $M$ é gerado em grau $j$ se $M \neq 0$ e $\Gamma^{(k)} M^{(j)}=M^{(j+k)}, \forall k$, e que $M$ é um módulo de Koszul se $M$ é um $\Gamma$-módulo finitamente gerado, graduado, gerado em grau zero que possue resolução linear, (cf. a definição 1.6), ou seja, $M$ tem uma $\Gamma$-resolução projetiva:

$$
\cdots \longrightarrow P_{(3)}(M) \stackrel{f_{3}}{\longrightarrow} P_{(2)}(M) \stackrel{f_{2}}{\longrightarrow} P_{(1)}(M) \stackrel{f_{1}}{\longrightarrow} P_{(0)}(M) \stackrel{f_{0}}{\longrightarrow} M \longrightarrow 0
$$

tal que $P_{(i)}(M)$ é gerado em grau $i$, para cada $i \geq 0$.

Em [GM,1] foi provado que $M$ é um módulo de Koszul, se e somente se $M$ tem $\Gamma$-resolução projetiva minimal, que satisfaz as condições abaixo:

1. $f_{i}: P_{(i)}=\underset{r}{\oplus} \Gamma e_{r} \longrightarrow P_{(i-1)}=\bigoplus_{t} \Gamma e_{t}$ (onde os $e_{r}^{\prime} s$ e $e_{t}^{\prime} s$ são idempotentes) é tal que $f_{i}\left(e_{r}\right) \in \Gamma^{(1)}$, para cada $e_{r} \in P_{(i)}$ e cada $i \geq 0$, com $i<\operatorname{pd} M$.

2. $r \cdot P_{(l)}=r^{2} \cdot P_{(l-1)} \cap P_{(l)}$, para $l=\operatorname{pd} M$. 
Uma propriedade interessante dos morfismos entre projetivos gerados em mesmo grau sobre álgebras como a álgebra $\Gamma$ definida acima, pode ser apresentada como se segue.

Lema 2.1 Sejam $P$ e $Q$ módulos projetivos, não-nulos, gerados em grau zero e seja $f: P \rightarrow Q$ morfismo graduado, não nulo. Se $f$ é um monomorfismo então $f$ é um monomorfismo que cinde.

Demonstração: Como $f$ é morfismo de grau zero temos que o morfismo $\bar{f}: \frac{P}{r P} \longrightarrow \frac{Q}{r Q}$, induzido por $f$, é não-nulo. Como $\frac{P}{r P}$ e $\frac{Q}{r Q}$ são isomorfos as componentes de grau zero de $P$ e $Q$, respectivamente, e $f$ é monomorfismo, temos que $\bar{f}$ também será. Logo, $\bar{f}$ cinde. Sendo $P$ e $Q$ módulos projetivos resulta que $f$ cinde.

Pode-se provar que as condições 1 e 2 , dadas acima, são equivalentes à dizer que, para cada $i>0, P_{(j)}$ é um somando direto da cobertura projetiva de $r P_{(j-1)}$, usando o lema que acabamos de apresentar. Com efeito, o monomorfismo $P_{j} \hookrightarrow r P_{(j-1)}$ se fatora pela cobertura projetiva de $r P_{(j-1)}$, definindo um monomorfismo entre projetivos gerados em mesmo grau. Segue, pelo lema acima, que este monomorfismo cinde.

Lembremos que uma álgebra graduada $\Gamma$, nas condições que assumimos neste capítulo, é uma álgebra de Koszul, se todo $\Gamma$-módulo simples é um módulo de Koszul. Além disso, de acordo com os resultados apresentados em [G-M, 1], vale que se $\Gamma$ é uma álgebra quadrática com dimensão global 2 então $\Gamma$ é uma álgebra de Koszul. Por outro lado, os autores daquele trabalho, provam que se $\Gamma$ é uma álgebra de Koszul então $\Gamma$ é uma álgebra quadrática. Como conseqüência destes resultados, eles concluem que nas álgebras de dimensão global 2, os conceitos Koszul e quadrática são equivalentes.

\section{2 Álgebras quadráticas de dimensão global finita}

Apresentaremos aqui, uma caracterização das álgebras quadráticas graduadas, que permitirá, como conseqüência, apresentar alguns outros resultados relativos a aquelas de dimensão global 2 e 3 . Além disso, poderemos 
obter uma caracterização parcial das álgebras de Koszul de dimensão global 3 .

Definição 2.2 Seja $\Gamma=k Q / I$ uma k-álgebra, onde $Q$ é uma aljava finita $e$ $I$ é um ideal admissivel e homogêneo. Definimos para cada $X \in \Gamma-\bmod$, a função numérica $\gamma$, dada por:

$$
\begin{aligned}
\gamma(X)= & \operatorname{dim}_{k} \operatorname{Hom}_{\Gamma}\left(I / I^{2}, X\right)-\operatorname{dim}_{k} \operatorname{Hom}_{\Gamma}\left(r P_{(1)}, X\right)+ \\
& +\operatorname{dim}_{k} \operatorname{Hom}_{\Gamma}\left(r^{2}, X\right)
\end{aligned}
$$

onde $P_{(1)}$ é a cobertura projetiva de $r$, o radical graduado de $\Gamma$.

Esta função permitiu provar a caracterização a qual nos referimos acima, através do seguinte teorema:

Teorema 2.3 Seja $\Gamma=k Q / I$, onde $Q$ é uma aljava finita e I é um ideal admissivel homogêneo. Seja $\gamma$ a função numérica definida acima. Então $\Gamma$ é uma álgebra quadrática se e somente se $\gamma(\Gamma / r)=0$.

Demonstração: Considerando a $\Gamma$-resolução projetiva minimal de $\Gamma / r$ fixada em $\left(^{*}\right)$, temos a seqüência curta exata

$$
0 \longrightarrow \Omega^{1}(r) \longrightarrow P_{(1)} \stackrel{\bar{f}_{1}}{\longrightarrow} r \longrightarrow 0
$$

onde $\bar{f}_{1}$ é a cobertura projetiva de $r$ que induz $f_{1}$.

Desde que $\frac{P_{1}}{r P_{1}} \cong \frac{r}{r^{2}}$ e por passagem aos núcleos, obtemos o seguinte diagrama comutativo:

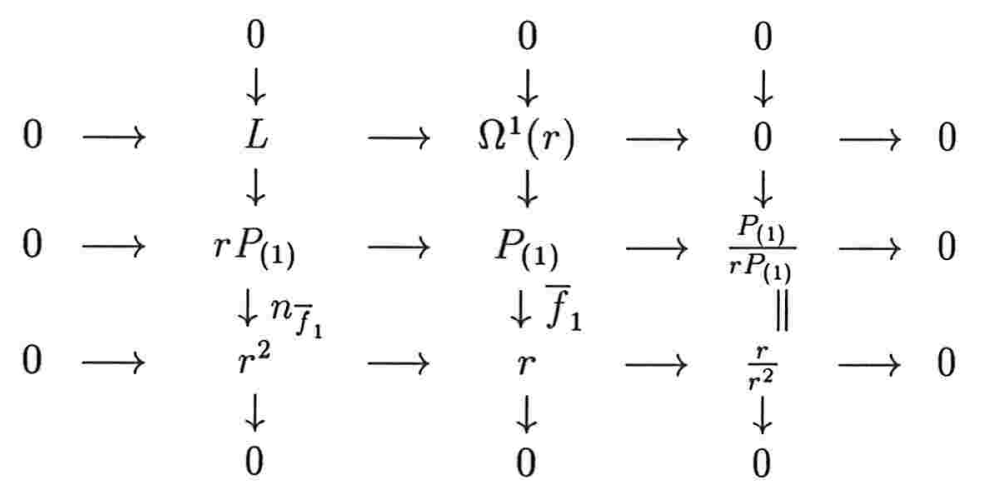


onde $n_{\bar{f}_{1}}$ foi obtida por passagem ao núcleo e $L=$ núcleo $\left(n \overline{f_{1}}\right)$. É facil ver que $L \cong \Omega^{1}(r)$.

Considerando a seqüência exata curta dada na $1 \underline{a}$ coluna à esquerda do diagrama (I), ou seja, a seqüência

$$
0 \longrightarrow \Omega^{1}(r) \longrightarrow r P_{(1)} \stackrel{n_{\bar{f}_{1}}}{\longrightarrow} r^{2} \longrightarrow 0
$$

e aplicando o funtor contravariante $\operatorname{Hom}_{\Gamma}(-, X)$, para $X \in \Gamma-\bmod$ a ela, obtemos a seguinte seqüência longa exata

$$
\begin{aligned}
0 & \longrightarrow \operatorname{Hom}_{\Gamma}\left(r^{2}, X\right) \longrightarrow \operatorname{Hom}_{\Gamma}\left(r P_{(1)}, X\right) \longrightarrow \\
& \longrightarrow \operatorname{Hom}_{\Gamma}\left(\Omega^{1}(r), X\right) \longrightarrow \operatorname{Ext}_{\Gamma}^{1}\left(r^{2}, X\right) \longrightarrow \\
& \longrightarrow \operatorname{Ext}_{\Gamma}^{1}\left(r P_{(1)}, X\right) \longrightarrow \operatorname{Ext}_{\Gamma}^{1}\left(\Omega^{1}(r), X\right) \longrightarrow \cdots
\end{aligned}
$$

Suponhamos, pois, que $\gamma(\Gamma / r)=0$.

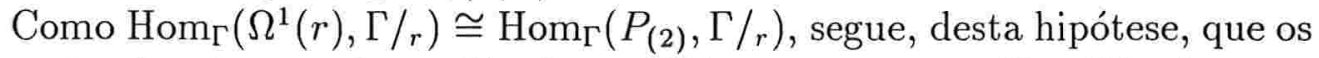
três primeiros termos da seqüência em (II), ao tomarmos $X=\Gamma / r$, formam uma seqüência exata curta. Do fato de que $\Gamma / r$ é um módulo semi-simples resulta que esta última seqüência induz uma outra seqüência exata curta, a saber, a seqüência

$$
0 \longrightarrow \operatorname{Hom}_{\Gamma}\left(\frac{r^{2}}{r^{3}}, \frac{\Gamma}{r}\right) \longrightarrow \operatorname{Hom}_{\Gamma}\left(\frac{r P_{(1)}}{r^{2} P_{(1)}}, \frac{\Gamma}{r}\right) \longrightarrow \operatorname{Hom}_{\Gamma}\left(\frac{\Omega^{1}(r)}{r \Omega^{1}(r)}, \frac{\Gamma}{r}\right) \longrightarrow 0
$$

Desta nova seqüência podemos concluir que $\frac{r^{2}}{r^{3}}$ é um somando direto de $\frac{r P_{(1)}}{r^{2} P_{(1)}}$, e portanto que $\frac{\Omega^{1}(r)}{r \Omega^{1}(r)} \cong \frac{P_{(2)}}{r P_{(2)}}$.

Deste fato resulta que $P_{(2)}$ é um somando direto da cobertura projetiva de $r P_{(1)}$. Como $\Gamma$ é 1-gerada então $r P_{(1)}$ é gerado em grau 2. Portanto, segue que $P_{(2)}$ é gerado em grau 2. Por [B], sabemos que $P_{(2)} \cong I / I^{2}$; e sendo $I$ um ideal graduado homogêneo temos então que $I$ é gerado em grau 2, donde segue que $\Gamma$ é um álgebra quadrática.

Reciprocamente, se I é um ideal quadrático então $P_{(2)}$ é gerado em grau 2. Portanto, $P_{(2)}$ é um somando da cobertura projetiva de $r P_{(1)}$ e, consequentemente, o morfismo $\operatorname{Hom}_{\Gamma}\left(-, \frac{\Gamma}{r}\right)\left(n_{\bar{f}_{1}}\right)$ é um epimorfismo. Logo, os três primeiros termos da seqüência em (II), para $X=\Gamma / r$, formam uma seqüência 
exata curta. De novo, usando que $\operatorname{Hom}_{\Gamma}\left(\Omega^{1}(r), \Gamma / r\right) \cong \operatorname{Hom}_{\Gamma}\left(P_{(2)}, \Gamma / r\right)$, segue então que $\gamma(\Gamma / r)=0$, como queríamos.

Corolário 2.4 Seja $\Gamma$ como no Teorema 2.3. Suponhamos que gldim $\Gamma=2$. Então $\Gamma$ é álgebra de Koszul $\Leftrightarrow \operatorname{Ext}_{1}^{1}\left(r^{2}, \Gamma / r\right) \cong \operatorname{Ext}_{\Gamma}^{1}\left(r P_{(1)}, \Gamma / r\right)$.

Demonstração: Desde que, para álgebras de dimensão global 2, os conceitos quadrática e Koszul são equivalentes, segue do Teorema 2.3, que $\Gamma$ é álgebra de Koszul se e somente se $\gamma(\Gamma / r)=0$. Afirmamos que $\gamma(\Gamma / r)=0$ se e somente se $\operatorname{Ext}_{\Gamma}^{1}\left(r^{2}, \Gamma / r\right) \cong \operatorname{Ext}_{\Gamma}^{1}\left(r P_{(1)}, \Gamma / r\right)$. Com efeito, observando o diagrama (II) apresentado na demonstração do Teorema 2.3 , e usando que gldim $\Gamma=2$, segue que é exata a seguinte seqüência longa

$$
\begin{aligned}
0 & \longrightarrow \operatorname{Hom}_{\Gamma}\left(r^{2}, \Gamma / r\right) \longrightarrow \operatorname{Hom}_{\Gamma}\left(r P_{(1)}, \Gamma / r\right) \longrightarrow \operatorname{Hom}_{\Gamma}\left(\Omega^{1}(r), \Gamma / r\right) \longrightarrow \\
& \longrightarrow \operatorname{Ext}_{\Gamma}^{1}\left(r^{2}, \Gamma / r\right) \longrightarrow \operatorname{Ext}_{\Gamma}^{1}\left(r P_{(1)}, \Gamma / r\right) \longrightarrow 0,
\end{aligned}
$$

pois $\Omega^{1}(r) \cong P_{(2)}$. Portanto, vale que $\gamma(\Gamma / r)=0 \Leftrightarrow$ a seqüência dada pelos 3 primeiros módulos na seqüência longa acima é exata $\Leftrightarrow \operatorname{Ext}_{\Gamma}^{1}\left(r^{2}, \Gamma / r\right) \cong$ $\operatorname{Ext}_{\Gamma}^{1}\left(r P_{(1)}, \Gamma / r\right)$.

\section{Observações:}

1. Se gldim $\Gamma=2$ então $\gamma(X) \geq 0$, para todo $X \in \Gamma-\bmod$.

Com efeito, a seqüência longa exata (II), que apresentamos na demonstração do Corolário 2.4, para X qualquer, nos diz que

$$
{ }_{\operatorname{Ext} \Gamma}^{1}\left(r P_{(1)}, X\right) \cong \frac{\operatorname{Ext}_{\Gamma}^{1}\left(r^{2}, X\right)}{H(X)}, \text { onde } H(X):=\frac{\left.\operatorname{Hom} \Gamma_{(2)}, X\right)}{\operatorname{Hom} \Gamma\left(r P_{(1)}, X\right) / P_{\text {нот } \Gamma}\left(r^{2}, X\right)} .
$$

Logo $\gamma(X)=\operatorname{dim}_{k} H(X) \geq 0$, como queríamos.

2. Se gldim $\Gamma=2$ e $r^{2}$ é um $\Gamma$-módulo projetivo então $\Gamma$ é uma álgebra de Koszul.

Do fato de ser $r^{2}$ um módulo projetivo e pela análise do diagrama (I), na demonstração do Teorema 2.3 , resulta que a seqüência exata curta

$$
0 \longrightarrow P_{2} \longrightarrow r P_{1} \longrightarrow r^{2} \longrightarrow 0
$$

cinde. Daí, segue que $P_{(2)}$ é gerado em grau 2 e, portanto, que $\Gamma$ é álgebra de Koszul. 
Os exemplos a seguir ilustram o comportamento da função $\gamma$, em $X=$ $\Gamma / r$, sendo que o exemplo 1 nos mostra que não vale a recíproca da observação 2 .

Exemplo 1 Seja $\Gamma$ a $k$-álgebra apresentada pela aljava e relações abaixo

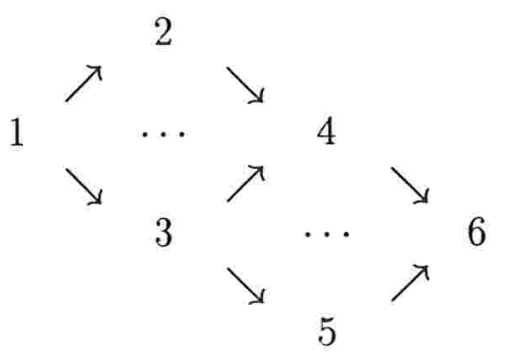

$\Gamma$ é claramente quadrática e é fácil ver que gldim $\Gamma=2$. Logo, é álgebra de Koszul.

Seja $M$ a $k$-representação abaixo:

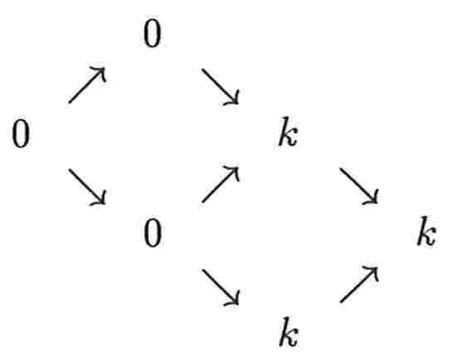

Um cálculo rápido mostra que $r P_{(1)}=P_{4} \oplus P_{6}^{3} \oplus M, r^{2}=P_{6}^{2} \oplus M$ e $P_{(2)}=$ $P_{4} \oplus P_{6}$. Note que $r^{2}$ não é um $\Gamma$-módulo projetivo.

Exemplo 2 Seja $\Gamma$ apresentada por:

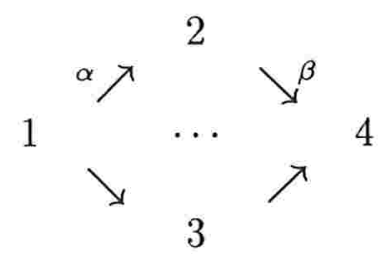

$\operatorname{com} \beta \alpha=0$.

$\Gamma$ é uma álgebra quadrática de dimensão global 2 (logo, é álgebra de Koszul). É fácil ver que $r P_{(1)}=r\left(P_{2} \oplus P_{3}\right)=S_{4}^{3}, r^{2}=S_{4}$, e $P_{(2)}=P_{4}^{2}$ e que $\gamma(\Gamma / r)=0$. Note que $r^{2}$ é um $\Gamma$-módulo projetivo. 
Exemplo 3 Seja $\Gamma$ apresentada por:

$$
1 \stackrel{\alpha}{\longrightarrow} 2 \stackrel{\beta}{\longrightarrow} 3 \stackrel{\gamma}{\longrightarrow} 4 \stackrel{\delta}{\longrightarrow} 5
$$

$\operatorname{com} \gamma \beta \alpha=0$. Temos que $\Gamma$ não é quadrática (logo, não é álgebra de Koszul).

Temos que $r^{2}=S_{3} \oplus P_{4} \oplus P_{5}, P_{(2)}=P_{4}$ e $r P_{(1)}=P_{3} \oplus P_{4} \oplus P_{5}$. É então claro que $\gamma(\Gamma / r)=1 \neq 0$ e $r^{2}$ não é um módulo projetivo.

Exemplo 4 Seja. $\Gamma$ apresentada por:

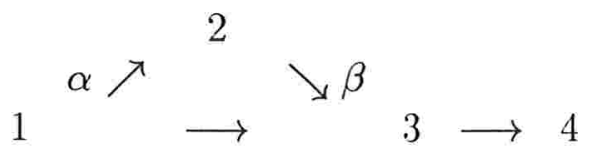

$\operatorname{com} \beta \alpha=0$.

$\Gamma$ é quadrática, de dimensão global $2 \mathrm{e}$, portanto, é álgebra de Koszul, e tal que $r^{2}=P_{4}^{2}, r P_{(1)}=S_{4} \oplus P_{3} \oplus P_{4}$ e $P_{(2)}=P_{3}$ e $\gamma(\Gamma / r)=0$.

Antes de continuarmos a expor nossos resultados, vamos apresentar o lema abaixo, que nos será útil no que segue neste capítulo.

Sejam $M$ e $N \quad \Gamma$-módulos graduados, ambos gerados em grau zero, e suponhamos que exista um epimorfismo graduado $f: M \rightarrow N$. Dadas as $\Gamma$ apresentações projetivas minimais de $M$ e $N$, podemos considerar o seguinte diagrama comutativo:

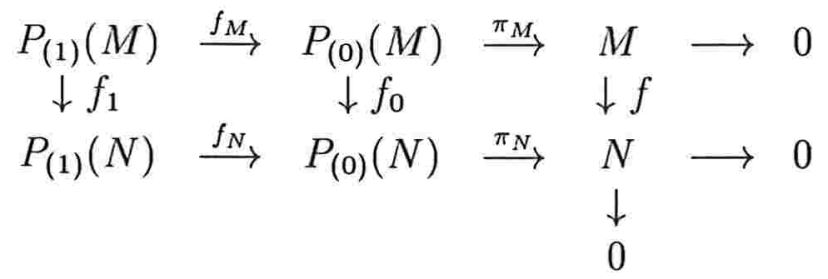

Lema 2.5 Com as hipóteses acima, vale que:

1. $f_{0}$ é um epimorfismo que cinde.

2. Se $N=\frac{M}{r M}$ e $M$ tem ambos apresentação linear, então $f_{1}$ é um monomorfismo que cinde.

Demonstração: 
1. Desde que $f$ é um epimorfismo, segue imediatamente que $f_{0}$ cinde.

2. Observamos que $f_{1}$ é um morfismo graduado, pois o diagrama que apresentamos é obtido por levantamentos na categoria gr $\Gamma$. Agora, se $N=M / r M$ e $M$ tem apresentação linear e então $P_{(1)}(M)$ e $P_{(1)}(N)$ são ambos gerados em grau 1. Mais ainda, temos que $P_{(1)}(M)$ é um somando direto da cobertura projetiva de $r P_{(0)}(M)$. Como $N$ é um módulo semi-simples, temos que $P_{(1)}(N)$ é a cobertura projetiva deste radical. Portanto, temos que $P_{(1)}(M)$ é um somando direto de $P_{(1)}(N)$. Segue que $f_{1}$ é um monomorfismo que cinde.

Proposição 2.6 Seja $\Gamma=k Q / I$ com $Q$ uma aljava finita, I um ideal homogêneo admissivel e gldim $\Gamma=3$. Suponhamos que $\Gamma$ seja quadrática $e$ $p d r^{2} \leq 1$. Então $\Gamma$ é uma álgebra de Koszul.

Demonstração: Consideremos a $\mathrm{Gr} \Gamma$-resolução projetiva de $\Gamma / r$ dada por:

$$
0 \longrightarrow P_{(3)} \stackrel{f_{3}}{\longrightarrow} P_{(2)} \stackrel{f_{2}}{\longrightarrow} P_{(1)} \stackrel{f_{1}}{\longrightarrow} P_{(0)}=\Gamma \longrightarrow \Gamma / r \longrightarrow 0
$$

Pela resolução projetiva minimal dada em $[\mathrm{B}]$, na seção 1.1 , pag. 462, para o semisimples $\Gamma / r$, vale que $P_{(2)} \cong I / I^{2}$. Como $\Gamma$ é quadrática segue que $P_{(2)}$ é gerado em grau 2. Assim, $\Gamma / r$ tem resolução linear de comprimento 2 e $r$ tem apresentação linear dada por $P_{(2)} \stackrel{f_{2}}{\longrightarrow} P_{(1)} \stackrel{\bar{f}_{1}}{\longrightarrow} r \longrightarrow 0$.

Consideremos, agora, o diagrama comutativo abaixo que é induzido pelas resoluções projetivas minimais de $r$ e $r / r^{2}$ :

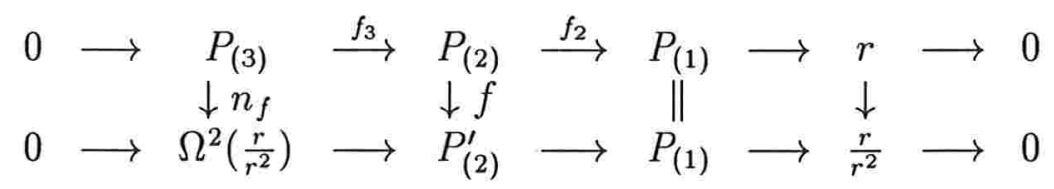

onde $n_{f}$ é obtida por passagem ao núcleo.

Do fato de que $\frac{r}{r^{2}} \in$ add $\left(\frac{\Gamma}{r}\right)$, segue que $\frac{r}{r^{2}}$ tem resolução linear de comprimento dois. Portanto, $\Omega^{2}\left(\frac{r}{r^{2}}\right)$ é um $\Gamma$-módulo gerado em grau 3 , já que $P_{(1)}$ é gerado em grau um.

Observamos que $P_{(2)}$ é um somando direto da cobertura projetiva de $r P_{(1)}$, ou seja, de $P_{(0)}\left(r P_{(1)}\right)=P_{(2)}^{\prime}$, uma vez que $P_{(2)}$ é gerado em grau 2. Por outro 
lado, o epimorfismo $r P_{(1)} \stackrel{n_{\mathcal{f}_{1}}}{\longrightarrow} r^{2}$, definido no diagrama (I) do Teorema 2.3, nos diz que $P_{(0)}\left(r^{2}\right)$ é também um somando direto de $P_{(1)}^{\prime}$. Além disso, a seqüência curta $0 \longrightarrow \Omega^{1}(r) \longrightarrow r P_{(1)} \stackrel{n_{\bar{f}_{1}}}{\longrightarrow} r^{2} \longrightarrow 0$, é exata, resultando então destes fatos que $P_{(1)}^{\prime}=P_{(0)}\left(r P_{(1)}\right)=P_{(2)} \oplus P_{(0)}\left(r^{2}\right)$.

Aplicando o lema 2.5 ao diagrama acima, temos que $f$ é um monomorfismo que cinde, e consequentemente, $n_{f}$ é um monomorfismo. Logo, podemos construir o seguinte diagrama comutativo:

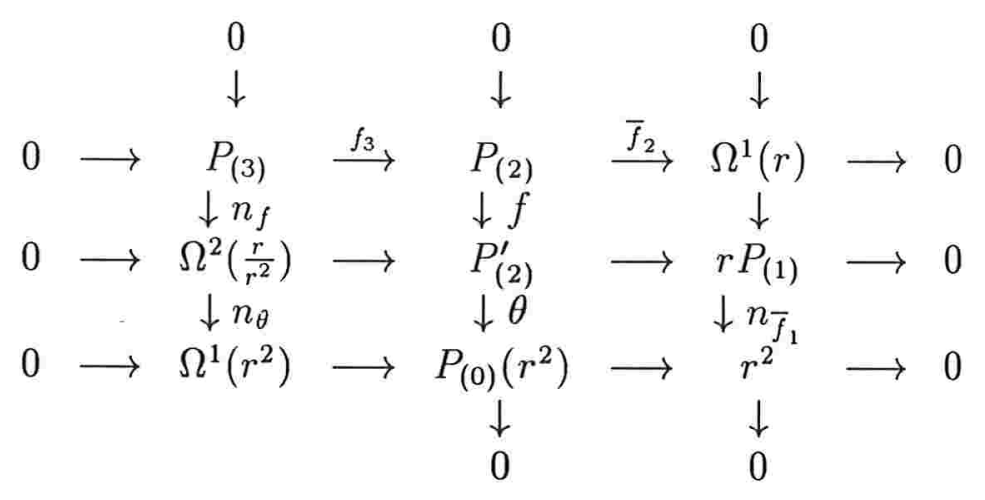

onde $n_{\theta}$ é obtida por passagem ao núcleo. Como $\bar{f}_{2}$ é um epimorfismo, segue que $n_{\theta}$ é um epimorfismo.

Seja a seqüência exata curta definida pela $1 \underline{\underline{a}}$ coluna à esquerda do diagrama acima, a saber:

$$
0 \longrightarrow P_{(3)} \stackrel{n_{f}}{\longrightarrow} \Omega^{2}\left(\frac{r}{r^{2}}\right) \stackrel{n_{\theta}}{\longrightarrow} \Omega^{1}\left(r^{2}\right) \longrightarrow 0
$$

Desde que, por hipótese, pd $r^{2} \leq 1$, temos que $\Omega^{1}\left(r^{2}\right)$ é um $\Gamma$-módulo projetivo. Se $\Omega^{1}\left(r^{2}\right)=0$ então $P_{(3)} \cong \Omega^{2}\left(\frac{r}{r^{2}}\right)$. Caso contrário, $P_{(3)}$ é um somando direto de $\Omega^{2}\left(\frac{r}{r^{2}}\right)$. Em ambas as situações, temos que $P_{(3)}$ é um $\Gamma$-módulo gerado em grau 3 . Portanto, $\Gamma$ é uma álgebra de Koszul.

\section{Observações:}

1. Seja $\Gamma=k Q / I$, onde $Q$ é uma aljava finita, $I$ é um ideal homogêneo quadrático e $3<\operatorname{gldim} \Gamma<\infty$. Se pdr $r^{2} \leq 1$, então $\Gamma / r$ tem resolução linear de comprimento 3 .

2. O exemplo a seguir mostra que a recíproca da Proposição 2.6 não é válida. 
Seja $\Gamma$ a $k$-álgebra apresentada por:

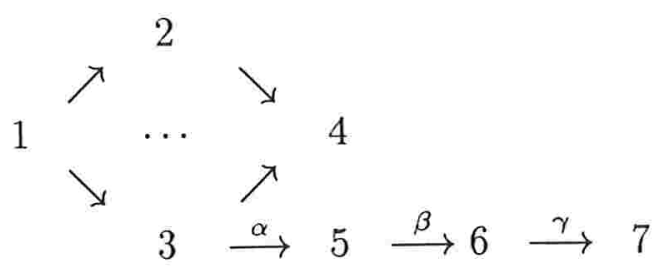

$\operatorname{com} \beta \alpha=\gamma \beta=0$.

Claramente $\Gamma$ é álgebra quadrática de gldim $\Gamma=3$ e pd $r^{2}=2$. Além disso, $\Gamma$ é álgebra de Koszul.

Em [GM,1], foi provado que se $\Gamma$ é uma álgebra de Koszul então $\Gamma$ é quadrática. Também, sobre álgebras de Koszul, temos que $r^{2}$ é um $\Gamma$-módulo de Koszul.

Estamos interessados, agora, em estudar a possibilidade destas duas propriedades acima, a saber, $\Gamma$ é uma álgebra quadrática e $r^{2}$ um $\Gamma$-módulo de Koszul, forneceram uma condição necessária e suficiente para termos uma álgebra de Koszul.

Suponhamos, então, que $\Gamma=k Q / I$, onde $Q$ é uma aljava finita, $I$ é um ideal admissível graduado e gldim $\Gamma=3$. Então, temos que pd $r^{2} \leq 2$.

Se $\operatorname{pd} r \leq 1$, pela Proposição 2.6, temos que $\Gamma$ é álgebra de Koszul. Assim, resta-nos analisar o caso em que $\mathrm{pd} r^{2}=2$. Para tanto, observamos que, como estamos assumindo gldim $\Gamma=3$, então pd $\frac{r}{r^{2}}=2$ ou 3 . Com efeito, suponhamos que seja pd $\frac{r}{r^{2}} \leq 1$. Como, claramente, não pode ocorrer de ser $\frac{r}{r^{2}}$ um $\Gamma$-módulo projetivo, então temos que pd $\frac{r}{r^{2}}=1$. Portanto, teríamos que $r P_{(1)} \cong P_{(2)}^{\prime}$ e através do diagrama (III) na demonstração da Proposição 2.6, concluiríamos que pd $r=1$, o que contraria o fato de ser pd $\frac{\Gamma}{r}=3$.

Ánalisemos, então, o caso em que pd $\frac{r}{r^{2}}=2$. Para apresentar nosso próximo resultado que tratará da situação em que pd $r^{2}=2$ e pd $\frac{r}{r^{2}}=2$, precisaremos apresentar um lema que nos auxiliará em sua demonstração.

Lema 2.7 Seja $\Gamma=k Q / I$, onde $Q$ é uma aljava finita e $I$ é um ideal quadrático, tal que gldim $\Gamma=3$. Seja $N=S_{1} \oplus \cdots \oplus S_{t}$ um módulo semisimples sobre $\Gamma$, tal que $S_{i} \not \approx S_{j}$, se $i \neq j$. Se pd $N=2$ então $P_{(2)}(N)$ é somando direto de $P_{(0)}\left(\Omega^{2}(\Gamma / r)\right)=P_{(2)}$. 
Demonstração: Imediata.

Proposição 2.8 Seja $\Gamma=k Q / I$, onde $Q$ é uma aljava finita, I é um ideal admissivel homogêneo e tal que gldim $\Gamma=3$. Suponhamos $\Gamma$ é quadrática $e$ que $p d r^{2}=2=p d\left(\frac{r}{r^{2}}\right)$. Se $r^{2}$ é um $\Gamma$-módulo de Koszul então $\Gamma$ é uma álgebra de Koszul.

Demonstração: Consideremos a seqüência exata curta

$$
0 \longrightarrow P_{(3)} \stackrel{n_{f}}{\longrightarrow} \Omega^{2}\left(\frac{r}{r^{2}}\right) \stackrel{n_{\theta}}{\longrightarrow} \Omega^{1}\left(r^{2}\right) \longrightarrow 0
$$

obtida no diagrama (III) da demonstração da Proposição 2.6.

Como pd $r^{2}=2$, podemos construir o seguinte diagrama comutativo:

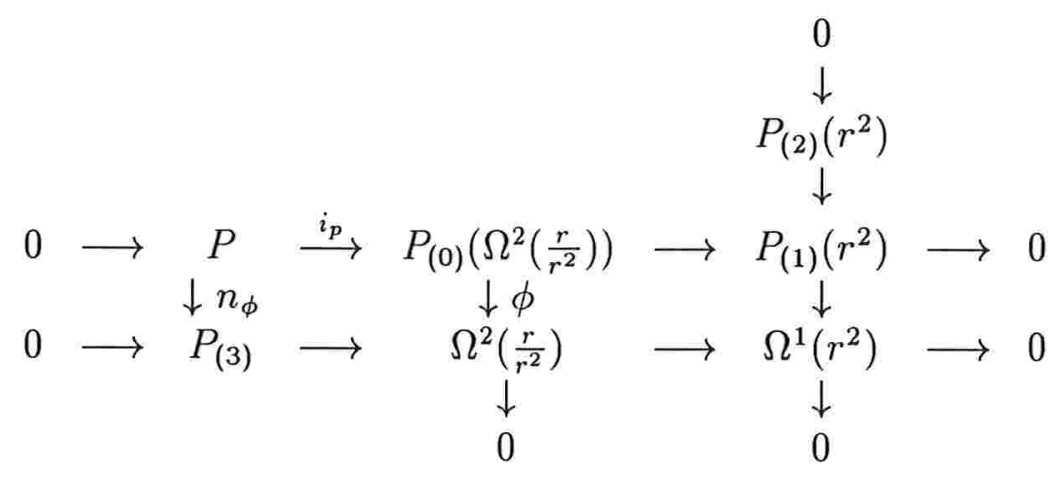

onde $n_{\phi}$ é obtida por passagem ao núcleo

Como pd $\frac{r}{r^{2}}=2$, temos que $\Omega^{2}\left(\frac{r}{r^{2}}\right) \cong P_{(0)}\left(\Omega^{2}\left(\frac{r}{r^{2}}\right)\right)$, ou seja, $\phi$ é um isomorfismo. Logo, $n_{\phi}$ é um monomorfismo, e pelo Lema da Serpente, o conúcleo de $n_{\phi}$ é isomorfo à $P_{(2)}\left(r^{2}\right)$.

Então, a seqüência exata curta $0 \longrightarrow P \stackrel{n_{\phi}}{\longrightarrow} P_{(3)} \longrightarrow P_{(2)}\left(r^{2}\right) \longrightarrow 0$, cinde. Ou seja, $P_{(3)}=P_{(2)}\left(r^{2}\right) \oplus P$.

Desde que, por hipótese, $r^{2}$ é um módulo de Koszul, segue que $P_{(2)}\left(r^{2}\right)$ é somando direto da cobertura projetiva de $r P_{(1)}\left(r^{2}\right)$. Pelo diagrama acima, temos que $P_{(1)}\left(r^{2}\right)$ é um somando direto de $P_{(0)}\left(\Omega^{2}\left(\frac{r}{r^{2}}\right)\right)=\Omega^{2}\left(\frac{r}{r^{2}}\right)$. Portanto, temos que $P_{(2)}\left(r^{2}\right)$ é um somando direto da cobertura projetiva de $r \Omega^{2}\left(\frac{r}{r^{2}}\right)$.

Como $\frac{r}{r^{2}}$ é um semi-simples temos que $\frac{r}{r^{2}}=S_{1}^{n_{1}} \oplus \cdots \oplus S_{t}^{n_{t}}$, onde podemos assumir que $S_{i} \not S_{j}$, se $i \neq j$. Aplicando o Lema 2.7 ao semi-simples $S_{1} \oplus$ 
$\cdots \oplus S_{t}$, que é um somando direto de $\Gamma / r$ de dimensão projetiva dois, obtemos, como conseqüência, que $\Omega^{2}\left(\frac{r}{r^{2}}\right) \in$ add $P_{(2)}$. Logo, $P_{(2)}\left(r^{2}\right)$ é gerado em grau 3 .

Por outro lado, observando que $P_{(0)}\left(\frac{r}{r^{2}}\right)=P_{(1)}$ que, por sua vez é gerado em grau um e que $\frac{r}{r^{2}}$ tem resolução linear de comprimento dois, segue que $P_{(2)}\left(\frac{r}{r^{2}}\right)$ é gerado em grau 3 , e portanto, teremos que $\Omega^{2}\left(\frac{r}{r^{2}}\right)$ é um $\Gamma$-módulo projetivo gerado em grau 3. Segue destes argumentos que $P$ é um módulo graduado gerado em grau 3.

Logo, temos que $P_{(3)}$ é gerado em grau 3 .

Portanto, $\Gamma$ é álgebra de Koszul.

Observamos que podemos ter $\Gamma$ uma álgebra de Koszul com gldim $\Gamma=3$ e pd $\frac{r}{r^{2}}=3$. O exemplo a seguir ilustra este fato. Seja $\Gamma$ a $k$-álgebra dada por:

$$
\begin{array}{lllllll}
1 & \longrightarrow & 2 & \stackrel{\alpha}{\longrightarrow} & 5 & \stackrel{\beta}{\longrightarrow} & 6 \stackrel{\gamma}{\longrightarrow}
\end{array}
$$

com as relações de comutatividade no quadrado e $\beta \alpha=0=\gamma \beta$.

É fácil ver que gldim $\Gamma=3$. Vale que pd $\frac{r}{r^{2}}=3$, uma vez que $S_{2}$, um dos simples do topo de $\frac{r}{r^{2}}$, tem resolução linear

$$
0 \longrightarrow P_{7} \longrightarrow P_{6} \longrightarrow P_{4} \oplus P_{5} \longrightarrow P_{2} \longrightarrow S_{2} \longrightarrow 0 \text {. }
$$

Portanto, podemos concluir que $\Gamma$ é álgebra de Koszul observando que todos os módulos simples tem resolução linear.

As Proposições 2.6 e 2.8 e as observações que fizemos antes de apresentar o Lema 2.7, permitiu-nos apresentar a seguinte caracterização parcial das álgebras de Koszul com dimensão global 3.

Teorema 2.9 Seja $\Gamma=k Q / I$ com $Q$ aljava finita, $I$ ideal graduado homogêneo, gldim $\Gamma=3$ e pd $\frac{r}{r^{2}}=2$. Então, $\Gamma$ é uma álgebra de Koszul se e somente se $\Gamma$ é quadrática com $r^{2}$ um módulo de Koszul.

Demonstração: Como observamos acima, o resultado é conseqüência das Proposições 2.6 e 2.8, e das observações que mencionamos. 


\subsection{Módulos de Koszul sobre as álgebras de Koszul de dimensão global 2}

Em [G-M,2], os autores provam na Proposição 6.1, que sobre álgebras hereditárias, um módulo $M$ é um módulo quasi-Koszul se, e somente se, $r M$ é um módulo projetivo. Este resultado motivou-nos a estabelecer o resultado seguinte, para as álgebras de Koszul.

Proposição 2.10 Seja $\Gamma$ uma k-álgebra graduada, 1-gerada, de decomposição básica, Koszul. Seja $M$ um $\Gamma$-módulo graduado, gerado em grau zero, tal que pd $M=1$. Se $r M$ é um módulo projetivo então $M$ é um módulo de Koszul.

Demonstração: Seja a gr $\Gamma$-resolução projetiva minimal de $M$ dada por:

$$
0 \longrightarrow P_{(1)} \stackrel{f}{\longrightarrow} P_{(0)} \stackrel{\pi}{\longrightarrow} M \longrightarrow 0
$$

Esta resolução induz a seqüência exata curta

$$
0 \longrightarrow P_{(1)} \stackrel{\bar{f}}{\longrightarrow} r P_{(0)} \stackrel{\tilde{\pi}}{\longrightarrow} r M \longrightarrow 0
$$

Como, por hipótese, $r M$ é projetivo, esta última seqüência cinde. Segue que $P_{(1)}$ é um somando direto de $r P_{(0)}$.

Por hipótese, temos que $M$ é gerado em grau zero, portanto $P_{(0)}$ é um módulo projetivo gerado em grau zero. Do fato de $\Gamma$ ser Koszul, segue que $r P_{(0)}$ é um módulo gerado em grau um. Logo, $P_{(1)}$ é um módulo gerado em grau um.

Portanto, $M$ é um módulo de Koszul.

O exemplo a seguir nos mostra que, sobre álgebras de Koszul $\Gamma$ com $1<\operatorname{gldim} \Gamma<\infty$, é possível encontrar módulos de Koszul de dimensão projetiva um cujo radical não é projetivo. Considere $\Gamma$ a $k$-álgebra dada por:

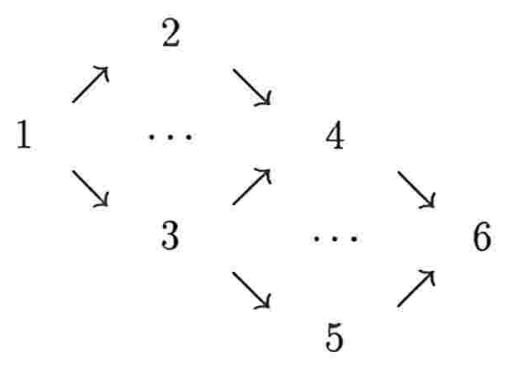


e considere $M$ o $\Gamma$-módulo cuja $k$-representação é:

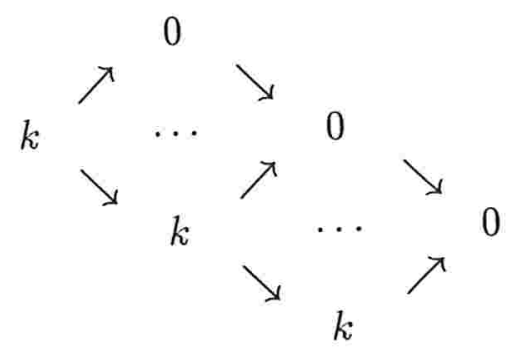

É fácil ver que $\operatorname{pd} M=1$. $M$ é um módulo de Koszul pois tem resolução projetiva $0 \longrightarrow P_{2} \longrightarrow P_{1} \longrightarrow M \longrightarrow 0$. No entanto, o radical de $M$, que é a $k$-representação abaixo, não é um módulo projetivo.

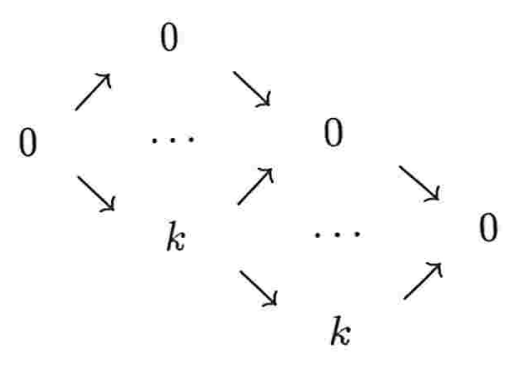

Nosso objetivo, agora, será estabelecer algumas condições que caracterizem os módulos de Koszul, sobre álgebras de Koszul de dimensão global 2. Assim, no que segue, estamos assumindo que gldim $\Gamma=2$.

Sejam $S$ um $\Gamma$-módulo simples, não-projetivo, e a apresentação linear de $S$ :

$$
P_{(1)} \stackrel{f}{\longrightarrow} P_{(0)} \longrightarrow S \longrightarrow 0
$$

Vamos escrever $P_{(1)}=\bigoplus_{m} P_{m}$, onde os $P_{m}$ são os projetivos indecomponíveis, para $m$ percorrendo um certo conjunto finito de índices. Escrevemos, também, $f=\left(f_{m}\right)_{m}$, onde $f_{m}$ é a restrição de $f$ a $P_{m}$.

Observamos que, se para cada $m$, a aplicação $f_{m}$ é um monomorfismo, o módulo conúcleo $f_{m}$ é um $\Gamma$-módulo de Koszul. De fato, a seqüência exata curta

$$
0 \longrightarrow P_{m} \stackrel{f_{m}}{\longrightarrow} P_{(0)} \longrightarrow \text { conuc } f_{m} \longrightarrow 0
$$

é uma resolução linear do $\Gamma$-módulo conuc $f_{m}$, o conúcleo de $f_{m}$, pois $S$ é módulo de Koszul. 
Isto que observamos acima, continua válido para qualquer somando direto de $P_{(1)}$. Notemos que, o fato de $f_{m}$ não ser um monomorfismo, implica que $r P_{m} \neq 0$.

O lema a seguir nos dá uma condição para que o conuc $f_{m}$ seja um $\Gamma$ módulo de Koszul no caso em que $f_{m}$ não é um monomorfismo. Denotaremos o módulo núcleo de $f_{m}$ por nuc $f_{m}$, e análogamente para qualquer morfismo.

Lema 2.11 Com a notação acima, seja $f_{m}$ uma função componente de $f$ tal que $f_{m}$ não é um monomorfismo. Então, conuc $f_{m}$ é um módulo de Koszul se e somente se nuc $f_{m}$ é um somando direto de nuc $f$.

Demonstração: Consideremos a seqüência exata longa

$$
0 \longrightarrow \operatorname{nuc} f_{m} \longrightarrow P_{m} \stackrel{f_{m}}{\longrightarrow} P_{(0)} \longrightarrow \operatorname{conuc} f_{m} \longrightarrow 0
$$

Como $P_{m}$ e $P_{(0)}$ são projetivos e gldim $\Gamma=2$, segue que nuc $f_{m}$ é um $\Gamma$-módulo projetivo. Portanto, a seqüência exata acima é uma resolução projetiva minimal para conuc $f_{m}$.

Consideremos o seguinte diagrama:

$$
\begin{aligned}
& 0 \longrightarrow \text { nuc } f_{m} \stackrel{\alpha}{\longrightarrow} P_{(0)}\left(r P_{m}\right) \\
& j_{m} \downarrow \\
& 0 \longrightarrow \operatorname{nuc} f \longrightarrow \bigoplus_{m} P_{(0)}\left(r P_{m}\right)
\end{aligned}
$$

onde $\alpha$ é o morfismo induzido pela inclusão nuc $f_{m} \hookrightarrow r P_{m}$ e $j_{m}$ a inclusão natural.

Como $\Gamma$ é Koszul, temos que $S$ é um módulo de Koszul, e portanto, nuc $f$ é um somando direto de $\bigoplus_{m} P_{(0)}\left(r P_{m}\right)$.

Logo, conuc $f_{m}$ é Koszul se e somente se nuc $f_{m}$ é somando direto de $P_{0}\left(r P_{m}\right)$ se e somente se nuc $f_{m}$ é somando direto de nuc $f$.

Com as observações que fizemos sobre a resolução de módulos simples sobre álgebras de Koszul e o lema enunciado acima, temos um critério para decidir quando os conúcleos das funções componentes da apresentação linear dos módulos simples, sobre álgebras de Koszul, são módulos de Koszul.

O resultado que segue, fornece uma caracterização homológica dos módulos com apresentação linear, sobre uma álgebra de Koszul, para que estes sejam módulos de Koszul. 
Temos vários exemplos em que a classe $\mathcal{L}(\Gamma)$ dos módulos com apresentação linear, contém estritamente a classe $\mathcal{K}(\Gamma)$, dos módulos de Koszul. Sabemos por [GMRSZ] que se $\Gamma$ é hereditária, elas coincidem. No caso de $\Gamma$ ser uma álgebra $B B$-inclinante (cf. cap. 3, seção 5 ), está claro que $\mathcal{L}(\Gamma)=\mathcal{K}(\Gamma)$. Para álgebras inclinadas em geral e álgebras graduadas de dimensão global 2, a inclusão é estrita, em geral, como mostra nosso próximo resultado.

Proposição 2.12 Seja $\Gamma=k Q / I$ onde $Q$ é uma aljava finita, I é um ideal admissivel homogêneo graduado, e tal que $\Gamma$ é Koszul de gldim $\Gamma=2$.

Seja $M$ um $\Gamma$-módulo graduado gerado em grau zero tal que pd $M=2$ e $M$ tem apresentação linear. Então, $M$ é um $\Gamma$-módulo de Koszul se e somente se $\Omega^{2}(M) \in$ add $\Omega^{2}(\Gamma / r)$, como módulos graduados.

Demonstraçâo: Consideremos o diagrama comutativo abaixo, que é induzido pelas gr $\Gamma$-resoluções projetivas de $M$ e $M / r M$

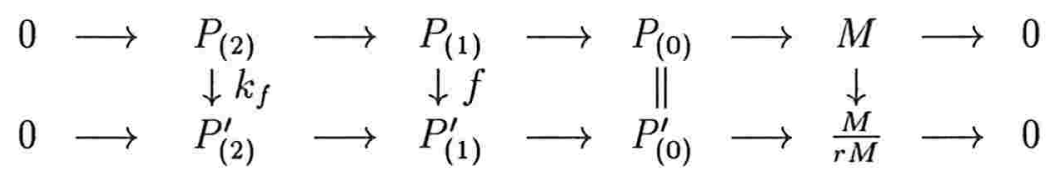

Como $M$ tem apresentação linear, segue pelo Lema 2.5, que $f$ é um monomorfismo que cinde. Portanto, temos que $k_{f}$ é um monomorfismo.

Mas $\Gamma$ é Koszul, e $\frac{M}{r M}$ é semi-simples gerado em grau zero, então $P_{(2)}^{\prime}$ é um somando direto da cobertura projetiva de $r P_{(1)}^{\prime}$.

Suponhamos que $M$ seja um módulo de Koszul. Então $P_{(2)}$ é somando direto da cobertura projetiva de $r P_{(1)}$ e portanto somando direto de $r P_{(1)}^{\prime}$ pois $f$ é monomorfismo que cinde, pelo Lema 2.5. Mas, $k_{f}$ é monomorfismo, portanto $P_{(2)}$ é um somando direto de $P_{(2)}^{\prime}$. Mas, temos que $P_{(2)}^{\prime} \in$ add $\Omega^{2}(\Gamma / r)$. Logo, o mesmo vale para $P_{(2)}$.

Reciprocamente, suponha que $P_{(2)}$ é somando direto de add $\Omega^{2}(\Gamma / r)$. Como observamos anteriormente, temos que $P_{(2)}^{\prime}$ é somando direto de add $\Omega^{2}(\Gamma / r)$. Do fato de $k_{f}$ ser monomorfismo, podemos concluir que $k_{f}$ cinde. Logo, $P_{(2)}$ será somando direto da cobertura projetiva de $r P_{(1)}$. Ou seja, $M$ é módulo de Koszul.

A proposição acima nos ensina um método de exibirmos módulos de Koszul sobre álgebras de Koszul de dimensão global 2, cuja apresentação seja conhecida. O exemplo a seguir ilustra este fato. 
Exemplo: Seja $\Gamma$ a $k$-álgebra dada por:

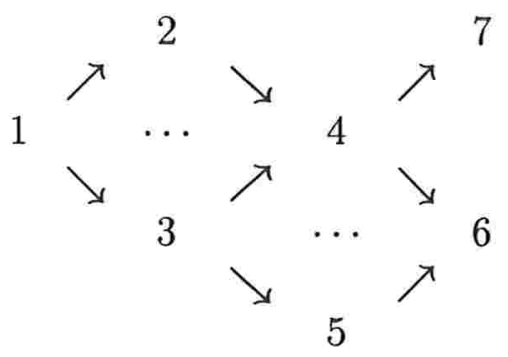

Temos $\Omega^{2}\left(S_{3}\right)=P_{6}$. Consideremos o $\Gamma$-módulo $M$ dado pela $k$-representação:

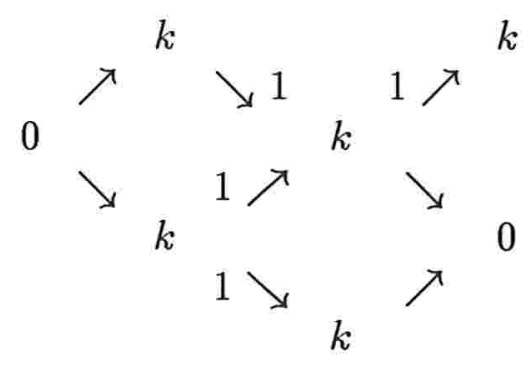

$M$ tem apresentação linear e $\Omega^{2}(M)=P_{6}$. Logo, $M$ é Koszul.

Com efeito, pois a $\Gamma$-resolução projetiva minimal de $M$ é:

$$
0 \longrightarrow P_{6} \longrightarrow P_{4} \oplus P_{6}^{2} \longrightarrow P_{2} \oplus P_{3} \longrightarrow M \longrightarrow 0
$$

Como, para $i=1,2, P_{(i)}$ é um somando direto da cobertura projetiva de $r P_{(i-1)}$, segue que esta resolução é linear.

Dado $M$ um módulo de Koszul de dimensão projetiva um, sobre uma álgebra de Koszul, é possível, sob certas condições, construir alguns módulos de Koszul sobre esta álgebra, a partir deste módulo $M$. Isto é mostrado na proposição abaixo. Este resultado tem um particular interesse quando tomamos $\Gamma$ uma álgebra inclinada Koszul e módulos de Koszul na classe de torção definida pelo módulo inclinante. Exemplos e mais detalhes sobre este caso serão expostos nos capítulos seguintes.

Proposição 2.13 Sejam $\Gamma$ uma álgebra de Koszul de dimensão global $2 e$ $M$ um $\Gamma$-módulo de Koszul, tal que pd $M=1$. 
Seja $M^{\prime}$ um submódulo graduado de $M$ tal que $p d M^{\prime}=1$, a inclusão $M^{\prime} \stackrel{j}{\hookrightarrow} M$, induz o monomorfismo $\frac{M^{\prime}}{r M^{\prime}} \stackrel{\bar{j}}{\longrightarrow} \frac{M}{r M}$ e $\frac{M}{M^{\prime}}$ não é um projetivo. Então, vale que

1. Se pd $\frac{M}{M^{\prime}}=1$, então $\frac{M}{M^{\prime}}$ e $M^{\prime}$ são módulos de Koszul.

2. $p d \frac{M}{M^{\prime}}=1$ se e somente se $M^{\prime}$ é módulo de Koszul.

3. Se pd $\frac{M}{M^{\prime}}=2$, então $\frac{M}{M^{\prime}}$ é Koszul, se e somente se $\Omega^{1}\left(M^{\prime}\right)$ é gerado em grau 2.

Demonstração: Sejam $P_{(0)}$ e $P_{(0)}^{\prime}$ as coberturas projetivas de $M$ e $M^{\prime}$, respectivamente. Do fato de, por hipótese, o monomorfismo $\bar{j}$ cindir, resulta que o monomorfismo $j^{\prime}: P_{(0)}^{\prime} \longrightarrow P_{(0)}$, induzido por $\bar{j}$, cinde. Mais ainda, o conúcleo deste morfismo é a cobertura projetiva de $\frac{M}{M^{\prime}}$.

Assim, considere o diagrama abaixo, obtido a partir das resoluções projetivas de $M, M^{\prime}$ e $\frac{M}{M^{\prime}}$, e que por isso, é comutativo:

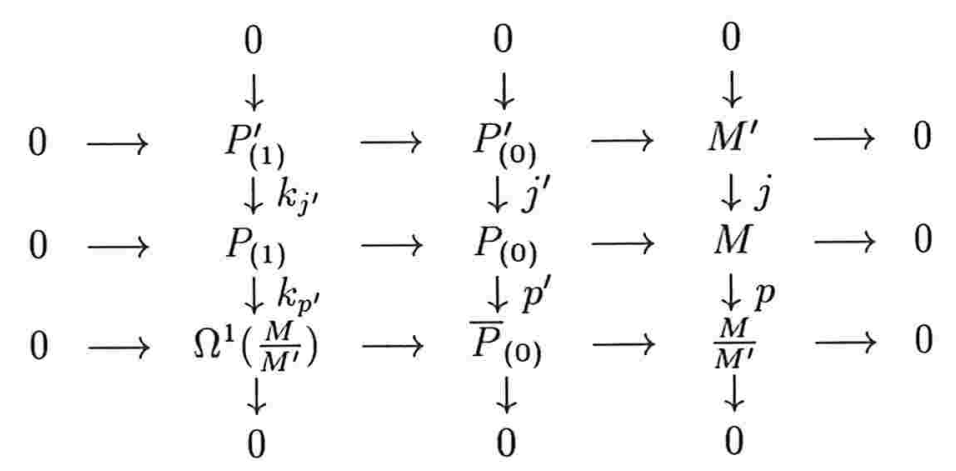

(1)Se pd $\frac{M}{M^{\prime}}=1$ então temos que $\Omega^{1}\left(\frac{M}{M^{\prime}}\right)$, é projetivo não-nulo. Então, $\Omega^{1}\left(\frac{M}{M^{\prime}}\right)$ é um somando direto de $P_{(1)}$, e portanto, um módulo gerado em grau um. Logo, $\frac{M}{M^{\prime}}$ é Koszul.

(2)Um argumento análogo ao acima, mostra que se pd $\frac{M}{M^{\prime}}=1$ então $M^{\prime}$ é Koszul.

Reciprocamente, se $M^{\prime}$ é Koszul então $P_{(1)}^{\prime}$ é gerado em grau um. Como $P_{(1)}$ também é gerado em grau um, $k_{j^{\prime}}$ é um monomorfismo, e $j^{\prime}$ é um monomorfismo que cinde, então $P_{(1)}^{\prime}$ é somando direto de $P_{(1)}$. Logo, a seqüência exata curta dada pela $1^{a} \underline{a}$ coluna à esquerda do diagrama, cinde, e portanto, $\Omega^{1}\left(\frac{M}{M^{\prime}}\right)$ tem que ser um somando direto de $P_{(1)}$, o que mostra que pd $\frac{M}{M^{\prime}}=1$. 
(3)Suponhamos que pd $\frac{M}{M^{\prime}}=2$. Então a seqüência exata curta

$$
0 \longrightarrow P_{(1)}^{\prime} \longrightarrow P_{(1)} \longrightarrow \Omega^{1}\left(\frac{M}{M^{\prime}}\right) \longrightarrow 0
$$

é uma resolução projetiva de $\Omega^{1}\left(\frac{M}{M^{\prime}}\right)$. Mas, pelos resultados em [GM,1], e que foram comentados no parágrafo 1.4 , é conhecido que $\frac{M}{M^{\prime}}$ é módulo de Koszul se e somente se $\Omega^{1}\left(\frac{M}{M^{\prime}}\right)[-1]$ também for, e isto ocorre, se e somente se $P_{(1)}^{\prime}$ é gerado em grau 2 .

Observamos que, nas condições da proposição acima, se assumirmos que $\frac{M}{M^{\prime}}$ é projetivo, segue que $M^{\prime}$ é módulo de Koszul, pois teriamos que $M=$ $M^{\prime} \oplus \frac{M}{M^{\prime}}$. Também, notemos que pode ocorrer ser $M^{\prime}$ um submódulo de $M$, nas condições fixadas pela proposição, e tal que pd $M^{\prime} \geq 1$.

Exemplo: O exemplo a seguir ilustra como funciona a Proposição 2.13 apresentada acima.

Seja $\Gamma$ a $k$-álgebra dada por:

$$
\begin{array}{lllll}
2 & \longleftarrow & 1 & \longrightarrow & 5 \\
\downarrow & \ddots & \downarrow & \ddots & \downarrow \\
4 & \longleftarrow & & \\
& & & \longrightarrow & 6 \\
& & & \\
& & & &
\end{array}
$$

com as relações de comutatividade. Seja $\Gamma$-módulo $M$, cuja $k$-representação é:

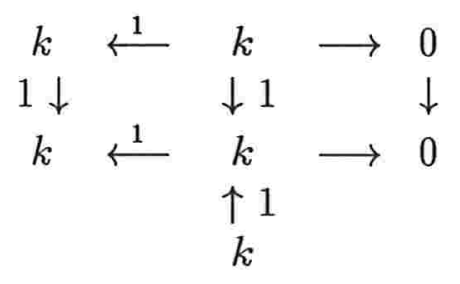

A resolução linear de $M$ é $0 \longrightarrow P_{3} \oplus P_{5} \longrightarrow P_{7} \oplus P_{1} \longrightarrow M \longrightarrow 0$, o que mostra que $M$ é Koszul. 
Consideremos o $\Gamma$-submódulo $M^{\prime}$ do módulo $M$, cuja $k$-representação é:

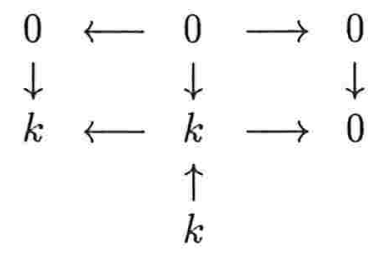

Notemos que $M^{\prime}$ não é Koszul, pois tem resolução projetiva $0 \longrightarrow P_{6} \longrightarrow$ $P_{7} \longrightarrow M^{\prime} \longrightarrow 0$, onde $P_{6}$ não é gerado em grau um. No entanto, $M^{\prime}$ se encontra nas hipóteses da Proposição 2.13 .

Observe que $\frac{M}{M^{\prime}}$ é o $\Gamma$-módulo cuja $k$-representação é:

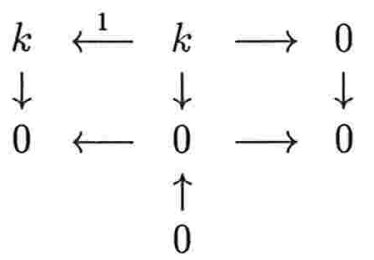

e cuja resolução projetiva $0 \longrightarrow P_{6} \longrightarrow P_{3} \oplus P_{5} \longrightarrow P_{1} \longrightarrow \frac{M}{M^{\prime}} \longrightarrow 0$ é linear, mostrando que $\frac{M}{M^{\prime}}$ é um $\Gamma$-módulo de Koszul com dimensão projetiva 2 .

Corolário 2.14 Seja $\Gamma$ uma álgebra de Koszul.

Sejam $M$ um $\Gamma$-módulo de Koszul e $M^{\prime}$ um submódulo graduado de $M$, ambos gerados em grau zero, tais que pd $M=p d M^{\prime}=1$ e $\frac{M}{M^{\prime}}$ não é um módulo projetivo. Então pd $M / M^{\prime}=1$ se e somente se $M^{\prime}$ é Koszul.

Demonstração: Imediata a partir da demonstração do item (2), na Proposição 2.13 , desde que $M$ e $M^{\prime}$ são ambos módulos gerados em grau zero, e portanto, a inclusão de $M^{\prime}$ em $M$, induz um morfismo de $\frac{M^{\prime}}{r M^{\prime}}$ em $\frac{M}{r M}$, que é um monomorfismo que cinde. Observamos que, como $\frac{M}{M^{\prime}}$ não é um módulo projetivo, então temos que $\operatorname{pd} \frac{M}{M^{\prime}} \geq 1$. 


\section{Capítulo 3}

\section{Álgebras de Koszul Brenner-Butler-inclinadas}

Neste capítulo, estaremos apresentando uma descrição completa das álgebras Brenner-Butler-inclinadas, centrada no resultado abaixo, que nos motivou a descrever a aljava, as relações e os simples desta álgebra.

Teorema: Toda álgebra BB-inclinada é uma álgebra de Koszul.

Estaremos, também, apresentando uma generalização deste tipo de álgebra, que também tem a propriedade de ser álgebra de Koszul.

\subsection{Preliminares}

Nesta seção introduziremos as notações, as definições e os fatos mais importantes da teoria que serão usados na apresentação de nossos resultados.

Seja $\Lambda=k Q$ a álgebra de caminhos sobre um corpo $k$, onde $Q$ é uma aljava finita, conexa e sem ciclos orientados. (Segue, pois, que $\Lambda$ é uma $k$-álgebra de dimensão finita hereditária).

Seja $P_{1}, P_{2}, \cdots, P_{n}$, uma lista completa dos $\Lambda$-módulos projetivos indecomponíveis, não isomorfos. Fixemos $S=S_{i}$ o $\Lambda$-módulo simples, associado ao vértice $i$ de $Q$. Suponhamos que $\tau^{-} S \neq 0$. Consideremos o $\Lambda$-módulo 
$T=\tau^{-} S \oplus \underset{j \neq i}{\bigoplus} P_{j}$. Desde que $\Lambda$ é hereditária, é claro que $\operatorname{pd} T \leq 1 \mathrm{e}$ $\operatorname{Ext}_{\Lambda}^{1}(T, T) \cong \operatorname{Ext}_{\Lambda}^{1}\left(\tau^{-} S, \tau^{-} S\right) \cong D \operatorname{Hom}_{\Lambda}\left(\tau^{-} S, S\right)=0$, pelas fórmulas de Auslander. Além disso, por construção, o número de somandos diretos indecomponíveis de $T$, não isomorfos, é igual ao número de classes de isomorfia dos $\Lambda$-módulos simples. Assim, pela definição 1.3 , temos que $T$ é um $\Lambda$-módulo inclinante.

Definição 3.1 Seja $S_{i}$ um módulo simples sobre $\Lambda=k Q$, onde $Q$ é uma aljava finita conexa, tal que $\tau^{-} S_{i} \neq 0$. Seja $T=\tau^{-} S_{i} \oplus \underset{j \neq i}{\oplus} P_{j}$ um $\Lambda$-módulo inclinante. No caso em que $S=S_{i}$ é um $\Lambda$-módulo projetivo, $T$ é chamado de APR-inclinante( $c f$. [ARS]).

Se $S=S_{i}$ não é um $\Lambda$-módulo projetivo, a álgebra $\Gamma=\operatorname{End}_{\Lambda}(T)^{o p}$ é chamada de álgebra Brenner-Butler inclinada ou simplesmente BB-inclinada.

É fato conhecido que, a classe dos módulos livre de torção $\mathcal{F}(T)=$ Cogen $(\tau T)$. Portanto, pela teoria de torção induzida pelo inclinante $T$, temos que $\mathcal{F}(T)=$ Cogen $(S)$, (cf. [AS,1]). Segue que ind $\mathcal{F}(T)=\{S\}$. Lembremos que, conforme observamos na seção 1.3, os projetivos indecomponíveis de $\Gamma$ são, a menos de isomorfismos, da forma $\operatorname{Hom}_{\Lambda}\left(T, P_{j}\right)$, para $j \neq i$ e $\operatorname{Hom}_{\Lambda}\left(T, \tau^{-} S\right)$.

Seja $\hat{S}=\operatorname{Ext}_{\Lambda}^{1}(T, S)$. Temos que $\hat{S}$ é um $\operatorname{End}_{\Lambda}(T)^{o p}$-módulo simples, pois vale que $\operatorname{Ext}_{\Lambda}^{1}(T, S) \cong \operatorname{DHom}_{\Lambda}(S, \tau T)=\operatorname{DHom}_{\Lambda}(S, S) \cong k$, pelas fórmulas de Auslander.

Além disso, sobre álgebras hereditárias, são bem conhecidas as seguintes propriedades:

1. Podemos ordenar os $\Lambda$-módulos projetivos indecomponíveis, de tal maneira que tenhamos $\operatorname{Hom}_{\Lambda}\left(P_{s}, P_{j}\right)=0$, se $s>j$.

2. Se $M$ é $\Lambda$-módulo indecomponível e $\operatorname{Hom}_{\Lambda}(M, P) \neq 0$ então $M$ é projetivo.

Portanto, se $S=S_{i}$ não é um projetivo, o item (2) nos diz que $\left(\tau^{-} S, P\right)=$ 0 , para todo $P$ projetivo. Por outro lado, se $S$ é um projetivo, como T é um inclinante, temos que $\operatorname{End}_{\Lambda}(T)^{o p}$ é uma algebra hereditária, logo, Koszul. 
Neste trabalho, estaremos interessados em analisar o caso em que $S$ não é um projetivo, para concluirmos que $\Gamma=\operatorname{End}_{\Lambda}(T)^{o p}$ é, também, uma álgebra de Koszul.

É conhecido que dados $\mathrm{M}$ e $\mathrm{N}$, módulos indecomponíveis sobre a álgebra hereditária $\Lambda$, não projetivos, tais que $\tau^{n} M \neq 0 \neq \tau^{n} N$, para algum $n \in \mathbb{Z}$, vale que $\operatorname{Hom}_{\Lambda}(M, N) \cong \operatorname{Hom}_{\Lambda}\left(\tau^{n} M, \tau^{n} N\right)$, (cf. em VIII, no[ARS]).

Como conseqüência desta assertiva temos que, se $S$ é um $\Lambda$-módulo simples, não projetivo e não injetivo, então $\operatorname{Hom}_{\Lambda}(S, S) \cong \operatorname{Hom}_{\Lambda}\left(\tau^{-} S, \tau^{-} S\right)$.

Conforme a observação anterior, fixemos uma ordem sobre os $\Lambda$-módulos projetivos indecomponíveis $P_{1}, \cdots, P_{n}$, de forma que $\left(P_{s}, P_{j}\right)=0$, se $s>j$.

Sejam $\hat{P}_{\hat{j}}=\left(T, P_{j}\right), j=1, \cdots, n$, com $j \neq i$, e $\hat{P}_{\hat{i}_{n}}=\left(T, \tau^{-} S\right)$, os $\Gamma$ módulos projetivos indecomponíveis. É claro que, para $j \neq i,\left(P_{s}, P_{j}\right) \cong$ $\left(\left(T, P_{s}\right),\left(T, P_{j}\right)\right)$. Como $S$ não é projetivo, temos que $\left(\hat{P}_{i_{n}}, \hat{P}_{\hat{j}}\right) \cong\left(\tau^{-} S, P_{j}\right)$ para $j \neq i$.

Assim, a ordenação dos $\Lambda$-módulos projetivos indecomponíveis, fixada acima, fornece uma ordenação sobre os projetivos indecomponíveis de $\Gamma$, de forma que

$$
\begin{gathered}
\left(\hat{P}_{\hat{s}}, \hat{P}_{\hat{j}}\right)=0, \text { se } \hat{s}>\hat{j} \quad(s, j \neq i) \\
\left(\hat{P}_{i_{n}}, \hat{P}_{\hat{j}}\right)=0, \text { com } j \neq i
\end{gathered}
$$

Lembrando que $P_{i}$ não é somando direto de $T$, e que estamos considerando, ao longo deste trabalho, módulos à esquerda, resulta que $\Gamma=\operatorname{End}_{\Lambda}(T)^{o p}=$ $\Gamma_{\Gamma} \Gamma=\bigoplus_{\substack{j \neq i \\ j=1}}^{n} \hat{P}_{\hat{j}} \oplus \hat{P}_{\hat{i}_{n}}$ e que

$$
\Gamma \cong\left[\begin{array}{cccccc}
\left(P_{1}, P_{1}\right) & 0 & 0 & \cdots & 0 & 0 \\
\left(P_{1}, P_{2}\right) & \left(P_{2}, P_{2}\right) & 0 & \cdots & 0 & 0 \\
\left(P_{1}, P_{3}\right) & \left(P_{2}, P_{3}\right) & \left(P_{3}, P_{3}\right) & \cdots & 0 & 0 \\
\vdots & \vdots & \vdots & \vdots & \vdots & \\
\left(P_{1}, P_{n}\right) & \left(P_{2}, P_{n}\right) & \left(P_{3}, P_{n}\right) & \cdots & \left(P_{n}, P_{n}\right) & 0 \\
\left(P_{1}, \tau^{-} S\right) & \left(P_{2}, \tau^{-} S\right) & \left(P_{3}, \tau^{-} S\right) & \cdots & \left(P_{n}, \tau^{-} S\right) & \left(\tau^{-} S, \tau^{-} S\right)
\end{array}\right]
$$

Das observações acima, temos que $\left(\tau^{-} S, P_{j}\right)=0,\left(\tau^{-} S, \tau^{-} S\right) \cong(S, S) \cong$ $k$, e $\left(P_{s}, P_{s}\right) \cong k$, para cada $s$, (pois $\Lambda$ é hereditária), segue que $\Gamma$ pode ser 
descrita como uma álgebra de matrizes, da seguinte forma:

$$
\Gamma \cong\left[\begin{array}{ccccc}
k & 0 & 0 & \cdots & 0 \\
\left(P_{1}, P_{2}\right) & k & 0 & \cdots & 0 \\
\left(P_{1}, P_{3}\right) & \left(P_{2}, P_{3}\right) & k & \cdots & 0 \\
\vdots & \vdots & \vdots & \vdots & \vdots \\
\left(P_{1}, \tau^{-} S\right) & \left(P_{2}, \tau^{-} S\right) & \left(P_{3}, \tau^{-} S\right) & \cdots & k
\end{array}\right]
$$

Considere $\left\{\mathcal{E}_{\hat{1}}, \mathcal{E}_{\hat{2}}, \ldots, \mathcal{E}_{\hat{i}_{n}}\right\}$ o sistema completo de idempotentes ortogonais primitivos de $\Gamma$ tal que $\Gamma=\Gamma \mathcal{E}_{\hat{i}_{n}} \oplus \cdots \oplus \Gamma \mathcal{E}_{\hat{1}}$. Comparando com a descrição de $\Gamma$, como uma álgebra de matrizes, dada acima, podemos após uma reordenação de índices, se necessária, identificar os $\Gamma$-módulos projetivos à esquerda, da seguinte maneira:

$$
\Gamma \mathcal{E}_{\hat{j}}=\hat{P}_{\hat{j}}=\left(T, P_{j}\right) \text { para } j \neq i, \Gamma \mathcal{E}_{\hat{i}_{n}}=\left(T, \tau^{-} S\right) .
$$

Assim, enquanto álgebra de matrizes, os $k$-espaços vetoriais que são as componentes dos $\Gamma$-módulos projetivos indecomponíveis, estão alinhadas em linhas.

A seguir, estaremos apresentando alguns lemas que nos descrevem os radicais dos projetivos $\left(T, P_{j}\right)$ sobre $\Gamma$, onde $j \neq i$ e fornecendo uma descrição de $\left(T, P_{i}\right)$, em termos dos módulos que pertencem à teoria de torção definida por $T$.

Lema 3.2 Sejam $l_{1}, l_{2}, \cdots, l_{t}$ os vértices predecessores imediatos de $i$ e $P_{j}$ um projetivo indecomponivel, com $j \notin\left\{l_{1}, \cdots, l_{t}, i\right\}$. Então $r_{\Gamma}\left(T, P_{j}\right) \stackrel{\Gamma-\underset{m o d}{\cong}}{\cong}$ $\left(T, r_{\Lambda} P_{j}\right)$.

Demonstração: Temos que $\left(T, P_{j}\right) \stackrel{(k)}{\cong} \bigoplus_{l \leq j}\left(P_{l}, P_{j}\right)$. Portanto, resulta que $r_{\Gamma}\left(T, P_{j}\right) \stackrel{k}{\cong} \bigoplus_{l<j}\left(P_{l}, P_{j}\right)$.

Mas, se $l<j$, temos que $\left(P_{l}, P_{j}\right) \cong\left(P_{l}, r_{\Lambda} P_{j}\right)$ e portanto $r_{\Gamma}\left(T, P_{j}\right) \cong$ $\bigoplus_{l<j}\left(P_{l}, r_{\Lambda} P_{j}\right) \cong\left(T, r_{\Lambda} P_{j}\right)$.

Como $r_{\Gamma}\left(T, P_{j}\right)$ é um $\Gamma$-submódulo de $\left(T, r P_{j}\right)$, pois a $j$-ésima componente de $\psi$, dada por $\psi_{j j}$ é nula, para toda $\psi \in r_{\Gamma}\left(T, P_{j}\right)$, segue pela igualdade acima que o isomorfismo que apresentamos é um isomorfismo de $\Gamma$-módulos. 
Lema 3.3 Seja $i$, o indice que fixamos acima. Então, vale que $\left(T, P_{i}\right) \cong$ $\left(T, r_{\Lambda} P_{i}\right)$.

Demonstração: Considere a seqüência exata curta $0 \longrightarrow r P_{i} \stackrel{\beta}{\longrightarrow} P_{i} \longrightarrow$ $\frac{P_{i}}{r P_{i}} \longrightarrow 0$. Aplicando o funtor $\operatorname{Hom}_{\Lambda}(T,-)$, obteremos imediatamente que $\left(T, P_{i}\right) \stackrel{\beta_{*}}{\cong}\left(T, r_{\Lambda} P_{i}\right)$, pois $\frac{P_{i}}{r P_{i}}$ é simples e $\left(T, \frac{P_{i}}{r P_{i}}\right)=0$.

Lema 3.4 Seja $P=\bigoplus_{s=1}^{t} P_{l_{s}}$, com $l_{1}, \ldots, l_{t}$ os vértices antecessores imediatos de i. Denotemos $r_{\Lambda} \stackrel{s=1}{P_{l_{s}}}=P_{i}^{m_{s}} \oplus P_{l_{s}}^{\prime}$, para cada $s=1, \ldots, t$, onde $P_{l_{s}}^{\prime}=$ $\oplus_{l^{\prime}} P_{l^{\prime}}^{m_{l^{\prime}}}$ com $l^{\prime}$ definidos pelos vértices sucessores imediatos de $l_{s}$, e $m_{s}, m_{l^{\prime}}$ o número de flechas de $l_{s}$ para $i$ e de $l_{s}$ para $l^{\prime}$, respectivamente. Então, $r_{\Gamma}\left(T, P_{l_{s}}\right) \cong\left(T, r_{\Lambda} P_{i}\right)^{m_{s}} \oplus\left(T, P_{l_{s}}^{\prime}\right)$, como $\Gamma$-módulos.

Além disso, se denotamos $P^{\prime}=\bigoplus_{s=1}^{t} P_{l_{s}}^{\prime}$, então $r_{\Gamma}(T, P)=\bigoplus_{s=1}^{t}\left(T, r_{\Lambda} P_{i}\right)^{m_{s}} \oplus$ $\left(T, P^{\prime}\right)$.

Demonstração: Pela identificação que exibimos, da álgebra $\Gamma$, como uma álgebra de matriz triangular inferior, é fácil ver que, fixado $s$, temos

$$
r_{\Gamma}\left(T, P_{l_{s}}\right)=\left\{\varphi: T \longrightarrow P_{l_{s}} / \varphi_{l_{s} l_{s}}: P_{l_{s}} \longrightarrow P_{l_{s}}, \text { é nula }\right\} \text {. }
$$

Porém, todo morfismo chegando em $P_{l_{s}}$, que não seja isomorfismo, se fatora pelo radical de $P_{l_{s}}$. Logo, resulta que $r_{\Gamma}\left(T, P_{l_{s}}\right)=\left(T, r_{\Lambda} P_{l_{s}}\right)$.

Segue disso que $r_{\Gamma}(T, P)=\oplus_{s=1}^{t} r_{\Gamma}\left(T, P_{l_{s}}\right)=\oplus_{s=1}^{t}\left[\left(T, r_{\Lambda} P_{i}\right)^{m_{s}}\right] \oplus\left(T, P^{\prime}\right)$.

O próximo resultado é uma conseqüência imediata dos lemas acima.

Lema 3.5 Sejam $\Gamma=\Gamma_{i}$ e $P$ um $\Lambda$-módulo projetivo. Então, $\operatorname{Hom}_{\Lambda}(T, P)$ tem radical projetivo.

Demonstração: Imediata pelos Lemas 3.2, 3.3, 3.4. 


\subsection{Os módulos simples sobre $\Gamma$}

Nesta seção estaremos descrevendo os $\Gamma$-módulos simples. Lembrando que o posto do grupo de Grothendieck de $\Lambda$ e de $\Gamma$ coincidem, poderemos exibir um conjunto completo das classes de isomorfia de tais módulos. No caso dos simples livres de torção, vamos indicar quais são os $\Lambda$-módulos $M$ tais que $\operatorname{Hom}_{\Lambda}(T, M)$ é um $\Gamma$-módulo simples. Como já observamos, o módulo simples de torção, é o módulo $\hat{S} \cong \operatorname{Ext}_{\Lambda}^{1}(T, S)$, para o qual estaremos exibindo, neste parágrafo, uma resolução projetiva minimal.

Para as descrições propostas, necessitamos conhecer a resolução projetiva minimal de $\tau^{-} S_{i}$ em $\Lambda$-mod. No entanto, aproveitaremos para, através desta resolução, provar que $\tau^{-} S_{i}$ é um módulo de Koszul.

Seja $0 \longrightarrow S \longrightarrow I_{i} \longrightarrow I_{1} \longrightarrow 0$ a coresolução injetiva minimal do $\Lambda$ módulo simples $S=S_{i}$, onde $I_{1}=I_{l_{1}}^{m_{1}} \oplus \cdots \oplus I_{l_{t}}^{m_{t}}$ tal que $l_{s}, s=1, \cdots, t$ são os vértices predecessores imediatos de $i$, e $m_{s}$ é o número de flechas de $l_{s}$ para i. Então, temos por [ARS], em 4.1.11,pag.107, que o topo de $\tau^{-} S=\operatorname{soc} I_{1}$.

Teorema 3.6 O $\Lambda$-módulo $\tau^{-} S$ é um módulo de Koszul.

Demonstração: Do fato de $\Lambda$ ser hereditária, temos que $(D S)^{*}=0$. Portanto, a seqüência curta

$$
0 \longrightarrow\left(D I_{i}\right)^{*} \longrightarrow\left(D I_{1}\right)^{*} \longrightarrow \tau^{-} S \longrightarrow 0
$$

é uma $\Lambda$-resolução projetiva de $\tau^{-} S$. Como $\left(D I_{j}\right)^{*}=\left(P_{j}\right)^{*}$, para todo vértice $j$ de $Q$, temos que a resolução é da forma

$$
0 \longrightarrow P_{i} \stackrel{f}{\longrightarrow} \bigoplus_{s=1}^{t} P_{l_{s}}^{m_{s}} \stackrel{\pi}{\longrightarrow} \tau^{-} S \longrightarrow 0
$$

onde $f$ é o morfismo induzido pela multiplicação pelas flechas que ligam os vértices $l_{1}, \ldots, l_{t}$ a $i$ na aljava de $\Lambda$.

Como $P_{i}$ é somando direto de $\operatorname{rad} \bigoplus_{j} P_{l_{j}}$, e $\Lambda$ é graduada, cada $P_{l_{s}}$ é um módulo projetivo gerado em grau zero, resultando que $\tau^{-} S$ é gerado em grau zero, e a resolução é linear. 
Consideremos, como já fizemos nas seções anteriores, $S_{l_{1}}, \ldots, S_{l_{t}}$ os módulos simples sobre $\Lambda$, não-isomorfos, associados aos vértices $l_{1}, \ldots, l_{t}$, antecessores imediatos de $i$. Observamos que $\frac{\tau^{-} S}{r_{\Lambda} \tau^{-S}}=\bigoplus_{S=1}^{t} S_{l_{s}}$, para $S=S_{i}$, conforme a demonstração do Teorema 3.6, acima.

Para nossos propósitos, precisaremos introduzir a noção de traço. Dados $M$ e $N$ módulos sobre a álgebra $\Lambda$, definimos o traço de $M$ em $N$, que denotamos por $\operatorname{tr}_{N}(M)$, como sendo o submódulo de $M$ gerado pelas imagens de todos os morfismos de $N$ em $M$.

Seja $Q_{s}$ a soma dos módulos projetivos indecomponíveis em $\Lambda$, não isomorfos entre si, e distintos de $P_{l_{s}}$ e $P_{i}$.

Proposição 3.7 Um conjunto dos representantantes das classes de isomorfia dos $\Gamma$-módulos simples, é o conjunto

$$
\left\{\operatorname{Hom}_{\Lambda}(T, M): M \in \mathcal{T}(T)\right\} \sqcup\left\{\hat{S}=\operatorname{Ext}_{\Lambda}^{1}(T, S)\right\}
$$

onde $M$ é um dos seguintes módulos:

1. $M \cong S_{j}$ com $j \neq i, j \notin\left\{l_{1}, \cdots, l_{t}\right\}$.

2. $M=M_{s}=\frac{P_{l_{s}}}{\operatorname{tr}_{Q_{s}}\left(P_{l_{s}}\right)}$, onde $s=1, \ldots, t$.

Demonstração:

1. Se $M$ é um módulo simples com $M \notin\left\{S_{l_{1}}, \cdots, S_{l_{t}}, S_{i}\right\}$ então teremos que $\operatorname{Hom}_{\Lambda}(T, M) \cong \operatorname{Hom}_{\Lambda}(P(M), M) \cong k$.

2. Se $M=M_{s}=\frac{P_{l_{s}}}{\operatorname{tr}_{Q_{s}}\left(P_{l_{s}}\right)}, s=1,2, \cdots, t$, então, desde que $\operatorname{tr}_{Q_{s}}\left(P_{l_{s}}\right)=$ $\oplus_{j \neq i} P_{j}^{m_{j}}$, com $P_{j}$ submódulo de $P_{l_{s}}$, e $m_{j} \geq 1$, temos que $M$ é um $\Lambda$ módulo cuja $k$-representação é tal que $M(i) \cong k^{m_{s}}$, onde $m_{s}=$ número de flechas que ligam o vértice $l_{s}$ ao vértice $i ; M\left(l_{s}\right)=k ; M(j)=$ 0 , para todo $j \neq l_{s}, j \neq i$ e $M\left(\alpha_{s, i}\right)$ é um monomorfismo, para cada flecha $\alpha_{s, i}$ que liga $l_{s}$ a $i$.

Por outro lado, $\operatorname{Hom}_{\Lambda}\left(P_{i}, \tau^{-} S_{i}\right)=0$, pois $P_{i}=\Omega^{1}\left(\tau^{-} S_{i}\right)$, conforme o Teorema 3.6. Portanto, $\operatorname{Hom}_{\Lambda}\left(\tau^{-} S, M_{s}\right)=0$. Logo, $\operatorname{Hom}_{\Lambda}\left(T, M_{s}\right)=$ $\operatorname{Hom}_{\Lambda}\left(P_{l s}, M_{s}\right) \cong k$. 
Portanto, temos que o conjunto definido no enunciado desta proposição, é formado por $\Gamma$-módulos simples. É claro que, os elementos desses conjuntos, por construção, são dois a dois, não isomorfos, então, ele contém $n$ elementos distintos e é por isso, um conjunto de representantes dos módulos simples de $\Gamma$.

Observação: Notemos que a $k$-representação de $M=\frac{P_{l_{s}}}{\operatorname{tr}_{Q_{s}}\left(P_{l_{s}}\right)}$, conforme notação adotada na Proposição 3.7, pode ser esboçada localmente, pelo seguinte grafo

$$
k \stackrel{f_{i j}}{\longrightarrow} k^{m_{s}}
$$

onde $m_{s}$ é o número de flechas de $l_{s}$ para $i$.

Então, temos que $M$ é módulo de Koszul se e sómente se $\Gamma$ é uma APRinclinante. Com efeito, como $r_{\Lambda} M \cong S_{i}^{m_{s}}$, onde $m_{s}$ é o número de flechas de $l_{s}$ para $i$ e de acordo com [G-M,2], sabemos que, sobre álgebras hereditárias, um módulo $M$ é Koszul se e somente se $r M$ é projetivo, resulta que $S_{i}$ é um simples projetivo.

Nosso próximo resultado apresenta a resolução projetiva minimal do $\Gamma$ módulo simples $\hat{S}$.

Proposição 3.8 Mantendo a notação definida acima, temos que a seqüência exata abaixo é uma $\Gamma$-resolução projetiva minimal do simples $\hat{S}$,

$$
0 \longrightarrow\left(T, r P_{i}\right) \stackrel{f_{*}}{\longrightarrow}(T, P)=\bigoplus_{s=1}^{t}\left(T, P_{l_{s}}^{m_{s}}\right) \stackrel{\pi_{*}}{\longrightarrow}\left(T, \tau^{-} S\right) \longrightarrow \hat{S} \longrightarrow 0
$$

Demonstração: Consideremos a resolução projetiva de $\tau^{-} S_{i}$, dada na demonstração do Teorema 3.6. Aplicando o funtor $\operatorname{Hom}_{\Lambda}(T,-)$, obtemos a seguinte seqüência longa exata de $\Gamma$-módulos

$0 \longrightarrow\left(T, P_{i}\right) \stackrel{f_{*}}{\longrightarrow}(T, P)=\bigoplus_{s=1}^{t}\left(T, P_{l_{s}}\right)^{m_{s}} \stackrel{\pi_{*}}{\longrightarrow}\left(T, \tau^{-} S\right) \longrightarrow \operatorname{Ext}_{\Lambda}^{1}\left(T, P_{i}\right) \longrightarrow 0$

onde $\pi_{*}=\operatorname{Hom}_{\Lambda}(T, \pi)=$ composição $\operatorname{com} \pi$.

Afirmamos que $\operatorname{Ext} t_{\Lambda}^{1}\left(T, P_{i}\right)=$ conuc $\pi_{*} \cong \hat{S}$. Vejamos porque. Como $\pi_{*}$ é morfismo sobre um projetivo indecomponível, temos que $\pi_{*}$ não é um epimorfismo, resultando, então, que $\operatorname{Im} \pi_{*} \subseteq r_{\Gamma}\left(T, \tau^{-} S\right)$. 
Por outro lado, seja $\varphi \in r_{\Gamma}\left(T, \tau^{-} S\right)$, digamos $\varphi=\left(\varphi_{1}, \varphi_{2}, \cdots, \varphi_{n}\right)$ com $\varphi_{n} \in\left(\tau^{-} S, \tau^{-} S\right)$, então temos que ter $\varphi_{n}=0$, pois $\left(\tau^{-} S, \tau^{-} S\right) \cong k$; segue que $\varphi$ é da forma $\varphi=\left(\varphi_{1}, \varphi_{2}, \cdots, \varphi_{n-1}, 0\right): T \longrightarrow \tau^{-} S$. Se considerarmos o diagrama:

$$
\begin{aligned}
& T^{\prime} \\
& \downarrow \bar{\varphi} \\
& P \stackrel{\pi}{\longrightarrow} \tau^{-} S \longrightarrow 0
\end{aligned}
$$

onde $T^{\prime}=\bigoplus_{j \neq i} P_{j}$ e $\bar{\varphi}=\left(\varphi_{1}, \cdots, \varphi_{n-1}\right) \in r_{\Gamma}\left(T, \tau^{-} S\right)$, então temos que $\bar{\varphi}$ se fatora por $\pi$, ou seja, existe $\psi^{\prime}: T^{\prime} \longrightarrow P$ tal que $\bar{\varphi}=\pi \psi^{\prime}$. Mas, $\psi^{\prime}$ se estende a um $\Gamma$-morfismo $\psi: T \longrightarrow P$ com $\psi_{n}: \tau^{-} S \longrightarrow P$, nulo, e tal que $\varphi=\pi \psi$. Logo, $\varphi \in \operatorname{Im} \pi_{*}$, ou seja, $\operatorname{Im} \pi_{*}=r_{\Gamma}\left(T, \tau^{-} S\right)$. Portanto, conuc $\pi_{*} \cong \hat{S}$, como queriamos mostrar.

Observamos que, nuc $\pi_{*}=\operatorname{Hom}_{\Lambda}\left(T, P_{i}\right)$. Mas, pelo lema 3.3 , temos que $\left(T, P_{i}\right)=\left(T, r_{\Lambda} P_{i}\right)$, que é um $\Gamma$-módulo projetivo. Segue que a seqüência longa exata acima é uma resolução projetiva minimal de $\hat{S}$.

\subsection{A descrição da aljava de $\Gamma$}

Nesta seção estaremos descrevendo a aljava das álgebras BB-inclinadas. Antes de apresentar nossos resultados, faremos alguns comentários, para esclarecer o que queremos que provar adiante.

Um esboço local da aljava de $\Gamma$ pode ser dada da maneira como mostraremos a seguir. Considere um esboço da aljava de $\Lambda, Q_{\Lambda}$, dada localmente em torno do vértice $i$, por:

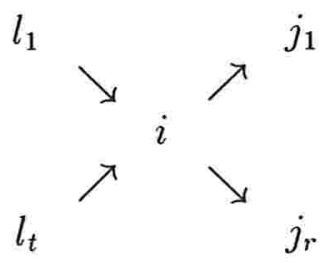

$\operatorname{com}\left(\alpha_{s}\right): l_{s} \longrightarrow i$ e $\left(\beta_{m}\right): i \longrightarrow j_{m}$ flechas unindo estes vértices, que estão sendo representadas por uma flecha única $\cdot \longrightarrow \cdot$ 
Sobre $Q(\Gamma)$, temos que $T$, como $\Lambda$-módulo à esquerda, produz localmente em torno dos vértices $\hat{i}_{n}$ e $\hat{j}_{m}$, o seguinte grafo orientado:

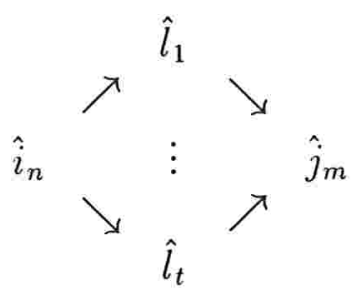

para cada $m=1, \ldots, r, \operatorname{com}\left(\delta_{s}\right): \hat{i}_{n} \longrightarrow \hat{l}_{s}$ flechas ligando estes vértices, representadas por $\cdot \longrightarrow \cdot$

Ou seja, o vértice $\hat{i}_{n}$, associado ao simples $S_{\hat{i}_{n}}=\hat{S}$, torna-se uma fonte, os vértices sucessores imediatos de $\hat{i}_{n}$ são os vértices $\hat{l}_{s}$, onde $l_{s} \operatorname{com} s=1, \cdots, t$ são os vértices predecesores imediatos de $i$, e os vértices sucessores imediatos destes $\hat{l}_{s}$ serão, entre outros, os vértices $\hat{j}_{m}$, onde $j_{m}$ com $m=1, \cdots, r$, são os vértices sucessores imediatos de $i$. No restante, a aljava permanece inalterada. Vejamos um exemplo para fixarmos nossas idéias.

Seja $\Lambda=k \tilde{D}_{5}$, o diagrama de Dynkin $\tilde{D}_{5}$, com a orientação dada por:

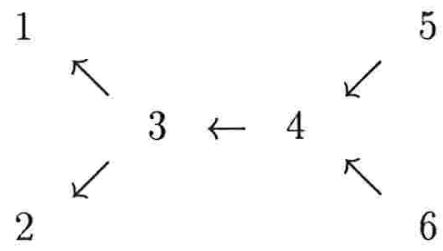

e tomemos $T=\tau^{-} S_{4} \oplus \underset{j \neq 4}{\bigoplus} P_{j}$, o módulo inclinante associado ao vértice 4 .

Então, temos que a aljava de $\Gamma$ é

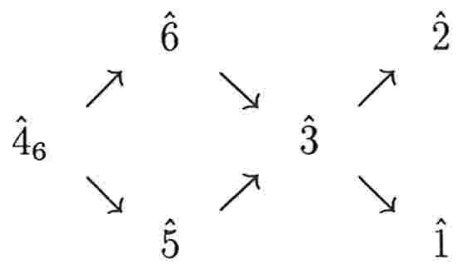

Os resultados que apresentamos a seguir demonstram as afirmações feitas acima. Lembremos que, como vimos no parágrafo 3.1 , vale que $r_{\Gamma}\left(T, P_{j}\right)=$ $\left(T, r_{\Lambda} P_{j}\right)$, para cada $j \neq i$ tal que $r_{\Lambda} P_{j} \in$ add $T$, e para cada $s=1, \cdots, t$, que $r_{\Gamma}\left(T, P_{l_{s}}\right)=\left(T, r_{\Lambda} P_{i}\right)^{m_{s}} \oplus\left(T, P_{l_{s}}^{\prime}\right)$, onde $r_{\Lambda} P_{l_{s}}=P_{i}^{m_{s}} \oplus P_{l_{s}}^{\prime}$. 
Proposição 3.9 Seja $j \neq i$ vértice em $Q(\Lambda)$, tal que o $\Lambda$-módulo projetivo indecomponível $P_{j}$ satisfaz " $P_{i}$ não é somando diveto de $r_{\Lambda} P_{j}$ e $P_{i}$ não é somando direto de $r_{\Lambda}^{2} P_{j} "$.

Então, \# flechas de $\hat{j}$ para $\hat{s}$ em $Q(\Gamma)=$ \# flechas de $j$ para $s$ em $Q(\Lambda)$, para cada $s \neq i$.

Demonstração: Para saber o número de flechas de $\hat{j}$ para $\hat{s}$, em $Q(\Gamma)$, precisamos calcular a dimensão do $k$-espaço vetorial $\mathcal{E}_{\hat{s}} \frac{r \Gamma}{r^{2} \Gamma} \mathcal{E}_{\hat{j}}$, (cf. Prop III.1.14 em [ARS]).

Pelos lemas da seção 3.1, cujos resultados foram relembrados logo acima, e da hipótese de que $j \notin\left\{i, l_{1}, \cdots, l_{t}\right\}$, temos que:

$$
\frac{r \Gamma}{r^{2} \Gamma} \mathcal{E}_{\hat{j}}=\frac{r\left(T, P_{j}\right)}{r^{2}\left(T, P_{j}\right)}=\frac{\left(T, r_{\Lambda} P_{j}\right)}{\left(T, r_{\Lambda}^{2} P_{j}\right)} .
$$

Agora, considere a seguinte seqüência exata de $\Lambda$-módulos:

$$
0 \longrightarrow r_{\Lambda}^{2} P_{j} \longrightarrow r_{\Lambda} P_{j} \longrightarrow \frac{r_{\Lambda} P_{j}}{r_{\Lambda}^{2} P_{j}} \longrightarrow 0
$$

Aplicando o funtor $\operatorname{Hom}_{\Lambda}(T,-)$, obtemos a seqüência exata longa de $\Gamma$ módulos, dada por:

$$
0 \longrightarrow\left(T, r_{\Lambda}^{2} P_{j}\right) \longrightarrow\left(T, r_{\Lambda} P_{j}\right) \longrightarrow\left(T, \frac{r_{\Lambda} P_{j}}{r_{\Lambda}^{2} P_{j}}\right) \longrightarrow \operatorname{Ext}_{\Lambda}^{1}\left(T, r^{2} P_{j}\right) \longrightarrow 0
$$

Mas, $\operatorname{Ext}_{\Lambda}^{1}\left(T, r_{\Lambda}^{2} P_{j}\right)=\operatorname{Ext}_{\Lambda}^{1}\left(\tau^{-} S_{i}, r_{\Lambda}^{2} P_{j}\right) \cong D \operatorname{Hom}_{\Lambda}\left(r_{\Lambda}^{2} P_{j}, S_{i}\right)$, pelas fórmulas de Auslander.

Como $P_{i}$ não é somando direto de $r_{\Lambda}^{2} P_{j}$, segue que $S_{i}$ não é somando direto de topo $r_{\Lambda}^{2} P_{j}$, logo $\left(r_{\Lambda}^{2} P_{j}, S_{i}\right)=0$, pois $r_{\Lambda}^{2} P_{j}$ é projetivo.

Segue pela exatidão da seqüência acima que

$$
\left(T, \frac{r_{\Lambda} P_{j}}{r_{\Lambda}^{2} P_{j}}\right) \cong \frac{\left(T, r_{\Lambda} P_{j}\right)}{\left(T, r_{\Lambda}^{2} P_{j}\right)}=\frac{r \Gamma}{r^{2} \Gamma} \mathcal{E}_{\hat{j}}
$$

Portanto,temos que $\operatorname{dim}_{k} \mathcal{E}_{\hat{s}} \frac{r \Gamma}{r^{2} \Gamma} \mathcal{E}_{\hat{j}}=\operatorname{dim}_{k}\left(P_{s}, \frac{r_{\Lambda} P_{j}}{r_{\Lambda}^{2} P_{j}}\right)=\#$ flechas de $j$ para $s$ em $Q(\Lambda)$.

Mais uma vez, observamos que a Proposição 3.9 mostra que o número de flechas de $\hat{j}$ para $\hat{s}$ em $Q(\Gamma)$, onde $s \neq i$ e $j \notin\left\{i, l_{1}, \cdots, l_{t}\right\}$ ou $j$ não é um 
antecessor imediato de algum $\operatorname{dos} l_{s}, \operatorname{com} s=1, \cdots, t$, é igual ao número de flechas de $j$ para $s$ em $Q(\Lambda)$.

Considere, agora, o seguinte esboço local para a aljava de $\Lambda$ :

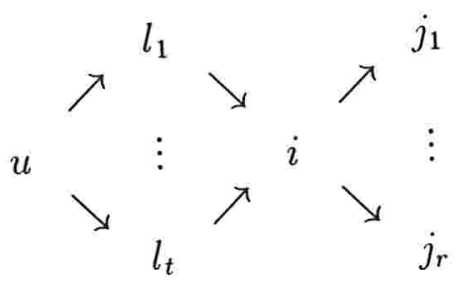

Precisamos analisar o que ocorre com os vértices $l_{1}, \cdots, l_{t}$ e os antecessores imediatos destes (como o vértice $u$ na figura), pois, para cada $l=l_{s}$, temos que $P_{i}$ é somando direto de $r_{\Lambda} P_{l}$ e para cada $u$ como acima, teremos $P_{i}$ é somando direto de $r_{\Lambda}^{2} P_{u}$ e portanto a proposição acima, não se aplica. Assim, precisaremos fazer uma análise distinta, para estes casos.

Proposição 3.10 Seja $l=l_{s}$ um vértice predececessor imediato de $i$ em $Q_{\Lambda}$. Denotemos $r P_{l_{s}}=P_{i}^{m_{s}} \oplus P_{l}^{\prime}$, onde $m_{s}$ é o número de flechas em $Q_{\Lambda}$ de $l_{s}$ para $i$, como no lema 3.4. Então, os vértices sucessores imediatos de $\hat{l}_{s}$ são os vértices $\hat{j}$, tais que $j$ é um sucessor imediato de $i$ ou $j$ é um sucessor imediato de $l_{s}$, distinto de $i$. Além disso, o número de flechas de $\hat{l}_{s}$ para $\hat{j}$ em $Q_{\Gamma}=m_{s}$. (o número de flechas de $i$ para $j$ em $\left.Q(\Lambda)\right)+$ o número de vezes que $S_{j}$ aparece no topo de $P_{l}^{\prime}$.

Demonstração: Sabemos que $\hat{P}_{\hat{\imath}}=\Gamma \mathcal{E}_{\hat{l}}=\left(T, P_{l}\right) \stackrel{k}{\cong} \bigoplus_{m \leq l}\left(P_{m}, P_{l}\right)$. Também, temos que $r_{\Lambda} \Gamma \mathcal{E}_{\hat{l}} \stackrel{\Gamma \text {-módulos }}{=}\left(T, r_{\Lambda} P_{i}\right)^{m_{s}} \oplus\left(T, P_{l}^{\prime}\right)$, onde temos que $r_{\Lambda} P_{l}=$ $P_{i}^{m_{s}} \oplus P_{l}^{\prime}$, como foi definido no Lema 3.4 .

Como $r_{\Lambda}^{2} P_{i}$ e $r_{\Lambda} P_{l}^{\prime} \in$ add $T$, os Lemas 3.2 e 3.4 , se aplicam e obtemos que $r_{\Gamma}\left(T, r_{\Lambda} P_{i}\right)=\left(T, r_{\Lambda}^{2} P_{i}\right)$, e que $r_{\Gamma}\left(T, P_{l}^{\prime}\right)=\left(T, r_{\Lambda} P_{l}^{\prime}\right)$. Logo,

$$
\begin{gathered}
\frac{r_{\Gamma} \Gamma \mathcal{E}_{\hat{l}}}{r^{2} \Gamma \mathcal{E}_{\hat{l}}}=\frac{\left(T, r_{\Lambda} P_{i}\right)^{m_{s}}}{r_{\Gamma}\left(T, r_{\Lambda} P_{i}\right)^{m_{s}}} \oplus \frac{\left(T, P_{l}^{\prime}\right)}{r_{\Gamma}\left(T, r_{\Lambda} P_{l}^{\prime}\right)} \cong\left(\frac{\left(T, r_{\Lambda} P_{i}\right)}{\left(T, r_{\Lambda}^{2} P_{i}\right)}\right)^{m_{s}} \oplus \frac{\left(T, P_{l}^{\prime}\right)}{\left(T, r_{\Lambda} P_{l}^{\prime}\right)} \\
\cong\left(T, \frac{r_{\Lambda} P_{i}}{r_{\Lambda}^{2} P_{i}}\right)^{m_{s}} \oplus\left(T, \frac{P_{l}^{\prime}}{r_{\Lambda} P_{l}^{\prime}}\right)
\end{gathered}
$$

pois $\operatorname{Ext}_{\Lambda}^{1}\left(T, r_{\Lambda}^{2} P_{i}\right)=0=\operatorname{Ext}_{\Lambda}^{1}\left(T, r_{\Lambda} P_{l}^{\prime}\right)$. Pela descrição dada na Proposicão 3.7 , temos que o topo de $r_{\Gamma} \mathcal{E}_{\hat{l}} \cong\left(\oplus_{m=1}^{r} \hat{S}_{\hat{j}_{m}}^{m_{j_{m}}}\right)^{m_{s}} \oplus\left(\oplus_{l^{\prime}} \hat{S}_{\hat{l}^{\prime}}^{m_{l^{\prime}}}\right)$, onde $l^{\prime}$ 
percorre o conjunto dos outros vértices sucessores de $l$, que são distintos de $i$, e $m_{l^{\prime}}$ é a multiplicidade do simples $\hat{S}_{\hat{l}^{\prime}}$ no topo de $r_{\Gamma} \mathcal{E}_{\hat{l}}$. Logo, $\operatorname{dim}_{k} \mathcal{E}_{\hat{j}} \frac{r \Gamma}{r^{2} \Gamma} \mathcal{E}_{\hat{\imath}} \neq 0$, para $j$ um sucessor imediato de $i$ ou $j$ tal que $S_{j}$ aparece no topo de $P_{l}^{\prime}$. Então, para estes $j$, o número de flechas de $\hat{l}$ para $\hat{j}$ em $Q(\Gamma)$ é igual a

$=m_{s} \cdot \operatorname{dim}_{k}\left(P_{j}, \frac{r_{\Lambda} P_{i}}{r_{\Lambda}^{2} P_{i}}\right)+\operatorname{dim}_{k}\left(P_{j}, \frac{P_{l}^{\prime}}{r_{\Lambda} P_{l}^{\prime}}\right)$

$=m_{s} \cdot$ o número de flechas de $i$ para $j$ em $Q(\Lambda)+$ número de vezes que $S_{j}$ aparece no topo de $P_{l}^{\prime}$.

Proposição 3.11 Seja u um antecessor imediato de alguns dos $l_{j}^{\prime}$ s, como no desenho abaixo:

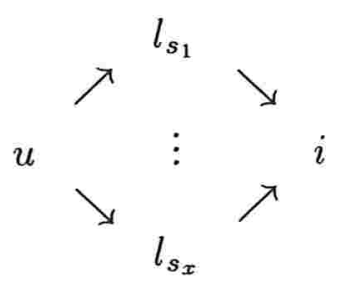

Então, os vértices sucessores imediatos de $\hat{u}$ em $Q(\Gamma)$ são os mesmos que em $Q(\Lambda)$, e o número de flechas que os liga em $Q(\Gamma)$, é o mesmo que os ligava em $Q(\Lambda)$.

Demonstração: Temos que $P_{i}$ é somando direto de $r_{\Lambda}^{2} P_{u}$. Assim, segue que $\frac{r_{\Gamma}\left(T, P_{u}\right)}{r_{\Gamma}^{2}\left(T, P_{u}\right)} \cong \frac{\left(T, r_{\Lambda} P_{u}\right)}{r_{\Gamma}\left(T, r_{\Lambda} P_{u}\right)}$. Seja $r_{\Lambda} P_{u}=\bigoplus_{s=1}^{x} P_{l_{s}}^{m_{u_{s}}} \oplus\left(\oplus_{u^{\prime}} \tilde{P}_{u^{\prime}}^{m_{u^{\prime}}}\right)$, onde $m_{u_{s}}$ é o número de flechas de $u$ para $l_{s}$ e $m_{u^{\prime}}$ é o número de flechas de $u$ para $u^{\prime} \notin\left\{l_{s_{1}} \cdots, l_{s_{x}}\right\}$. Então, temos que

$$
\begin{aligned}
& \frac{\left(T, r_{\Lambda} P_{u}\right)}{r_{\Gamma}\left(T, r_{\Lambda} P_{u}\right)}=\bigoplus_{s=1}^{x}\left(\frac{\left(T, P_{l_{s}}\right)}{r_{\Gamma}\left(T, P_{l_{s}}\right)}\right)^{m_{u_{s}}} \oplus \bigoplus_{u}^{\prime}\left(\frac{\left(T, \tilde{P}_{u^{\prime}}\right)}{r_{\Gamma}\left(T, \tilde{P}_{u^{\prime}}\right)}\right)^{m_{u^{\prime}}} \\
& =\bigoplus_{s}\left(T, M_{l_{s}}\right)^{m_{u_{s}}} \oplus \underset{u^{\prime}}{\bigoplus^{\prime}}\left(T, S_{u^{\prime}}\right)^{m_{u^{\prime}}}
\end{aligned}
$$

conforme a Proposição 3.7, do parágrafo anterior.

Se $j \neq u^{\prime}$ ou $j \notin\left\{l_{s_{1}}, \cdots, l_{s_{x}}\right\}$ então $\mathcal{E}_{j} \frac{r \Gamma}{r^{2} \Gamma} \mathcal{E}_{\hat{u}}=0$, pois o topo de $r_{\Gamma} \hat{P}_{\hat{u}}=$ $\bigoplus_{l_{s}}\left(\hat{S}_{\hat{l}_{s}}\right)^{m_{u_{s}}} \oplus \underset{u^{\prime}}{\bigoplus^{\prime}}\left(\hat{S}_{\hat{u}^{\prime}}\right)^{m} u_{u^{\prime}}$. 
Então, temos que, para cada $u^{\prime}$ definido acima, fixado,

$\operatorname{dim}_{k} \mathcal{E}_{\hat{u}^{\prime}} \frac{r_{\Gamma} \hat{P}_{\hat{u}}}{r_{\Gamma}^{2} \hat{P}_{\hat{u}}}=$ o número de vezes que $\hat{S}_{\hat{u}^{\prime}}$ aparece no topo de $r_{\Gamma} \hat{P}_{\hat{u}}$ $=$ o número de vezes que $S_{u^{\prime}}$ aparece no topo de $r_{\Lambda} P_{u}$ $=$ o número de flechas de $u$ para $u^{\prime}$ em $Q(\Lambda)$

E para cada $l \in\left\{l_{s_{1}}, \cdots, l_{s_{x}}\right\}$, temos que

$$
\begin{aligned}
\operatorname{dim}_{k} \mathcal{E}_{i} \frac{r_{\Gamma} \hat{P}_{\hat{u}}}{r_{\Gamma}^{2} \hat{P}_{\grave{u}}} & =\text { o número de vezes que } \hat{S}_{\hat{l}} \text { aparece no topo de } r_{\Gamma} \hat{P}_{\hat{u}} \\
& =\text { o número de flechas de } u \text { para } l \text { em } Q(\Lambda)
\end{aligned}
$$

Finalmente, observamos que $\left(\hat{P}_{\hat{i}_{n}}, \hat{P}_{\hat{j}}\right)=0$, para cada $j \neq i$, e da resolução projetiva de $\hat{S}$, que $\oplus_{s=1}^{t} \hat{P}_{\hat{l}_{s}}^{m_{s}}$ é a cobertura projetiva de $r_{\Gamma} \hat{P}_{\hat{i}_{n}}$. Assim, temos que $\hat{i}_{n}$ é uma fonte de $Q_{\Gamma}$, e que as extremidades das flechas que saem de $\hat{i}_{n}$ são os vértices $\hat{l}_{1}, \cdots, \hat{l}_{t}$. Além disso, temos que o número de flechas de $\hat{i}_{n}$ para $\hat{l}_{s}$ é o mesmo que o de $l_{s}$ para $i$. Com efeito, fixado $s=1, \cdots, t$, temos que o número de flechas de $\hat{i}_{n}$ para $\hat{l}_{s}=$ número de vezes que $\hat{S}_{\hat{l}_{s}}$ aparece no topo de $r_{\Gamma} \hat{P}_{\hat{i}_{n}}=$ o número de vezes que $S_{l_{s}}$ aparece no topo de $\tau^{-} S=o$ número de flechas de $l_{s}$ para $i$.

Assim, obtemos que a aljava de $Q_{\Gamma}$ é da forma descrita no início deste parágrafo.

\section{4 Álgebras Brenner-Butler-Koszul}

Neste parágrafo, estaremos interessados em demonstrar o principal resultado deste capítulo, a saber, que toda álgebra BB-inclinada é uma álgebra de Koszul. Para tanto, além de usarmos os resultados que apresentamos anteriormente, precisaremos descrever as aplicações entre alguns dos projetivos indecomponíveis de $\Gamma=\Gamma_{i}$, que são multiplicação por flechas. Seguindo a estes resultados, poderemos descrever as relações que estão definidas sobre $\Gamma$, o que faremos, logo após aos resultados que mencionamos acima. 
Sabemos que $\left(T, P_{i}\right) \cong\left(T, r_{\Lambda} P_{i}\right)$, pelo Lema 3.3. Para nossos propósitos, vamos explicitá-lo. Considere o morfismo de $\Gamma$-módulos:

$$
\left(T, r_{\Lambda} P_{i}\right) \stackrel{\beta_{*}}{\longrightarrow}\left(T, P_{i}\right)
$$

definido por $\beta_{*}(\varphi)=\beta \cdot \varphi$, para cada $\varphi \in\left(T, r_{\Lambda} P_{i}\right)$, onde $\beta=\left(\left(\beta_{1}^{v_{1}}\right)_{1 \leq u_{1} \leq v_{j_{1}}}\right.$, $\left.\cdots,\left(\beta_{r}^{v_{r}}\right)_{1 \leq v_{r} \leq v_{j_{m}}}\right)$ tal que $\beta_{m}^{v_{m}}$ é o morfismo definido pela multiplicação pela

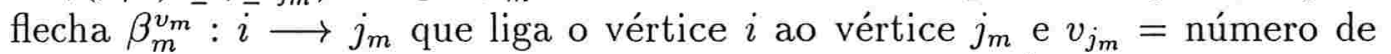
flechas entre estes vértices, para cada $m=1, \cdots, r$. É claro que $\beta$ é um monomorfismo. Pela identificação que apresentamos no Lema 3.3, segue que $\beta_{*}$ é um isomorfismo.

Seja $\hat{S}_{\hat{l}_{s}}$ o simples de $\Gamma$ associado ao vértice $\hat{l}_{s}$, onde $s=1, \cdots, t$, como convencionamos ao longo dos parágrafos anteriores. Pela Proposição 3.7, temos que $\hat{S}_{\hat{l}_{s}}=\operatorname{Hom}_{\Lambda}\left(T, M_{s}\right)$, onde $M_{s}$ tem $k$-representaçao que pode ser esboçada, localmente, pelo seguinte grafo

$$
k \stackrel{f_{i l s}}{\longrightarrow} k^{m_{l s}}
$$

onde $m_{l_{s}}$ é o número de flechas de $l_{s}$ para $i$. É fácil verificar que a resolução projetiva de $M_{s}$ é a seqüência exata curta $0 \longrightarrow r P_{i}^{m_{l_{s}}} \oplus P_{l}^{\prime} \stackrel{g=\left(g_{1}, g_{2}\right)}{\longrightarrow} P_{l_{s}} \longrightarrow$ $M_{s} \longrightarrow 0$, onde $r P_{l_{s}}=P_{i}^{m_{l_{s}}} \oplus P_{l}^{\prime}$ e o morfismo $g_{1}$ é dado pela multiplicação pela soma dos caminhos $\sum_{m, v_{m}, u_{s}} \beta_{m}^{v_{m}} \alpha_{s}^{u_{s}}$, que ligam o vértice $l_{s}$ aos vértices $j_{1}, \cdots, j_{m}$, passando por $i$, onde $\beta_{m}^{v_{m}}$ é a flecha que liga o vértice $i$ ao vértice $j_{m}$, como definimos acima, e $\alpha_{s}^{u_{s}}$ é a flecha que liga $l_{s}$ ao vértice $i$, para $u_{s}=1, \cdots, m_{l_{s}}$, onde $m_{l_{s}}$ é o número de flechas que ligam $l_{s}$ a $i$.

Aplicando o funtor $\operatorname{Hom}_{\Lambda}(T,-)$ a resolução projetiva de $M_{s}$, exibida acima, obtemos a seguinte seqüência exata curta

$$
0 \longrightarrow\left(T, r P_{i}^{m l_{s}} \oplus P_{l}^{\prime}\right) \stackrel{\left(g_{1_{*}}, g_{2_{*}}\right)}{\longrightarrow}\left(T, P_{l_{s}}\right) \longrightarrow\left(T, M_{s}\right)=\hat{S}_{\hat{l}_{s}} \longrightarrow 0
$$

que é a resolução projetiva minimal de $\hat{S}_{\hat{l}_{s}}$. Segue que cada função componente de $g_{1 *}$ é definida pela multiplicação por uma flecha que liga o vértice $\hat{l}_{s}$ ao vértice $\hat{j}_{m}$, para cada $m=1, \cdots, r$, ou seja, é a composição com o $\Lambda$-morfismo definido pela multiplicação pelo caminho $\beta_{m}^{v_{m}} \alpha_{s}^{u_{s}}$, fixados $m$ e $s$.

Vamos denotar estas flechas de $Q_{\Gamma}$, que definem o morfismo $g_{1 *}$, por $\gamma_{l_{s}, j_{m}}^{u_{s}, v_{m}}$, onde $s=1, \cdots, t, m=1, \cdots, r, 1 \leq u_{s} \leq m_{l_{s}}, 1 \leq v_{m} \leq v_{j_{m}}$, e tal que $m_{l_{s}}=$ número de flechas de $l_{s}$ para $i$, e $v_{j_{m}}=$ número de flechas de $i$ para $j_{m}$. 
Consideraremos, agora, a resolução projetiva dos $\Gamma$-módulos simples $\hat{S}_{\hat{j}}$, onde $j \notin\left\{i, l_{1}, \cdots, l_{t}\right\}$, dada por $0 \longrightarrow\left(T, r P_{j}\right) \stackrel{i_{\bullet}}{\longrightarrow}\left(T, P_{j}\right) \longrightarrow \hat{S}_{\hat{j}} \longrightarrow 0$. Com a apresentação dada pela Proposição 3.8 , juntamente com estas, que apresentamos acima, obtivemos o resultado a seguir.

Proposição 3.12 Seja $\Gamma=\Gamma_{i}$ uma álgebra BB-inclinante. Então, com a apresentação de $\Gamma$ dada como enunciamos acima, temos que $\Gamma$ é uma álgebra quadrática.

Demonstração: Consideremos $\mathcal{E}_{\mathfrak{j}_{1}}, \cdots, \mathcal{E}_{\hat{j}_{r}}$ os idempotentes de $\Gamma$, pertencentes ao sistema completo de idempotentes ortogonais primitivos de $\Gamma$, (como descrevemos em 3.1), e tais que $\oplus_{m=1}^{r}\left(\Gamma \mathcal{E}_{\hat{j}_{m}}\right)^{v_{m}}=\left(T, r_{\Lambda} P_{i}\right)$, onde cada $v_{j_{m}}$, é o número de vezes que $\hat{S}_{\hat{j}_{m}}$ aparece no topo de $\left(T, r_{\Lambda} P_{i}\right)$, como descrevemos anteriormente.

Seja a resolução projetiva minimal de $\hat{S}$, que foi obtida e apresentada na Proposição 3.8

$$
0 \longrightarrow\left(T, r P_{i}\right) \stackrel{f_{*}}{\longrightarrow}(T, P)=\bigoplus_{s=1}^{t}\left(T, P_{l_{s}}^{m_{l_{s}}}\right) \stackrel{\pi_{*}}{\longrightarrow}\left(T, \tau^{-} S\right) \longrightarrow \hat{S} \longrightarrow 0
$$

Com a identificação que apresentamos acima, para os módulos $\left(T, P_{i}\right)$ e $\left(T, r P_{i}\right)$, segue que $f_{*}$ é um $\Gamma$-morfismo definido pela composição por $f \beta$. Pelo que apresentamos no Teorema 3.6, temos que $f$ é o $\Lambda$-morfismo definido em cada função componente, pela multiplicação pela flechas que ligam os vértices $l_{s}$ ao vértice $i$, para $s=1, \cdots, t$. Assim, vamos considerar as $\alpha_{s}^{u_{s}}: l_{s} \longrightarrow i$, que são as flechas que ligam $l_{s}$ ao vértice $i$, onde $s=1, \cdots, t$ e $1 \leq u_{s} \leq m_{l_{s}}$, com $m_{l_{s}}=$ número de flechas de $l_{s}$ para $i$, como definimos nas observações feitas acima. Denotando o $\Lambda$-morfismo definido pela multiplicação pela flecha $\alpha_{s}^{u_{s}}$, com a mesma notação, obteremos que a matriz do morfismo $f$ é

$$
f=\left[\begin{array}{c}
\left.\left(\alpha_{1}^{u_{1}}\right)_{1 \leq u_{1} \leq m_{l_{1}}}\right) \\
\vdots \\
\left(\alpha_{t}^{u_{t}}\right)_{1 \leq u_{t} \leq m_{l_{t}}}
\end{array}\right]
$$

Então, o morfismo $f \beta$ está definido por uma matriz cujas entradas são as composições com os caminhos $\beta_{m}^{v_{m}} \alpha_{s}^{u_{s}}$, apresentados acima. Em particular, o morfismo restrição de $f \beta$ a $\left(T, P_{j_{m}}\right)^{v_{m}}$, para cada $m=1, \cdots, r$, que 
denotaremos por $f \beta_{m}$, é dado pela matriz

$$
\left[\begin{array}{cccc}
\beta_{m}^{1} \cdot \alpha_{1}^{1} & \beta_{m}^{2} \cdot \alpha_{1}^{1} & \cdots & \beta_{m}^{v_{j_{m}}} \cdot \alpha_{1}^{1} \\
\cdots & \cdots & \cdots & \cdots \\
\beta_{m}^{1} \cdot \alpha_{1}^{m_{l_{1}}} & \beta_{m}^{2} \cdot \alpha_{1}^{m_{l_{1}}} & \cdots & \beta_{m}^{v_{j_{m}}} \cdot \alpha_{1}^{m_{l_{1}}} \\
\vdots & \vdots & \vdots & \vdots \\
\beta_{m}^{1} \cdot \alpha_{t}^{m_{l_{t}}} & \beta_{m}^{2} \cdot \alpha_{t}^{m_{l_{t}}} & \cdots & \beta_{m}^{v_{j_{m}}} \cdot \alpha_{t}^{m_{l_{t}}}
\end{array}\right]
$$

Portanto, temos que cada entrada desta matriz é definida pela multiplicação pelo caminho $\beta_{m}^{v_{m}} \cdot \alpha_{s}^{u_{s}}$ em $Q_{\Lambda}$. Segue das observações e afirmações que fizemos acima, antes de enunciar este resultado, que cada morfismo $\left(\beta_{m_{c}}^{v_{m}} \cdot \alpha_{s}^{u_{s}}\right)_{*}$ é um $\Gamma$-morfismo definido pela multiplicação pela flecha $\gamma_{l_{s}, j_{m}}^{u_{s}, v_{m}}$, que liga o vértice $\hat{l}_{s}$ ao vértice $\hat{j}_{m}$.

Decorre destes argumentos, que o morfismo $(f \beta)_{*}$ tem cada uma de suas funções componentes definidas pela multiplicação por flechas de $Q_{\Gamma}$. Como $\pi_{*}$ é composição com morfismos que são multiplicação por flechas e como $\pi_{*} f_{*}=0$, segue que $\Gamma$, com a apresentação dada, é uma álgebra quadrática.

Como conseqüência da Proposição 3.12, obtemos o seguinte resultado:

Teorema 3.13 Toda álgebra Brenner-Butler inclinada é Koszul.

Demonstração: Pela Proposição 3.12, temos que $I$ é ideal quadrático. Mas, como $\Gamma=\Gamma_{i}$ tem dimensão global dois, segue, por [GM,1], que $\Gamma$ é uma álgebra de Koszul.

A seguir, apresentaremos as relações que estão definidas sobre $\Gamma$, e que decorrem dos resultados e da apresentação que obtivemos acima.

Seja $\tilde{\psi} \in\left(T, r_{\Lambda} P_{i}\right)$. Temos que $\left(\pi_{*} \circ f_{*}\right)(\tilde{\psi})=0$. Mas, se $\tilde{\psi}$ é um morfismo induzido pela multiplicação por uma soma de caminhos em $Q(\Gamma)$ com término nos vértices $\hat{j}_{1}, \ldots, \hat{j}_{r}$, segue que $\left(\pi_{*} \circ f_{*}\right)(\tilde{\psi})$ representa uma relação em $Q(\Gamma)$, começando em $\hat{i}_{n}$ e terminando nestes vértices.

Temos que $f_{*}(\tilde{\psi})=(f \circ \beta)(\tilde{\psi})$ onde $f$ e $\beta$, são os morfismos definidos acima, e tais que as funções componentes de $f \beta$ estão definidas pela multiplicação pelas flechas de $Q_{\Gamma}$, dadas por $\gamma_{l_{s}, j_{m}}^{u_{s}, v_{m}}$, como definimos nas observações que fizemos antes de apresentar a Proposição 3.12. 
Vimos que $\Lambda$-morfismo $\pi: \bigoplus_{s=1}^{t}=P_{l_{s}}^{m_{l_{s}}} \longrightarrow \tau^{-} S$ induz o $\Gamma$-morfismo $\pi_{*}$ que apresenta o $\Gamma$-módulo simples $\hat{S}$. Segue que $\pi_{*}=\left(\left(\delta_{1}^{u_{1}}\right)_{1 \leq u_{1} \leq m_{l_{1}}}, \cdots\right.$, $\left.\left(\delta_{t}^{u_{t}}\right)_{1 \leq u_{t} \leq m_{l_{t}}}\right)$, onde cada função componente é o morfismo definido pela multiplicação pela flecha $\left(\delta_{s}^{u_{s}}\right)$ que liga o vértice $\hat{i}_{n}$ ao vértice $\hat{l}_{s}$ em $Q(\Gamma)$.

Assim, temos que as entradas da matriz $\pi_{*}(f \beta)_{*}$, quando consideradas como elementos de $k Q(\Gamma)$, definem o ideal das relações de $\Gamma$. Em particular, para cada $m=1, \cdots, r$ fixado, temos que

$$
\begin{aligned}
& \pi_{*}\left(f \beta_{m}\right)_{*}= \\
& {\left[\delta_{1}^{1}\left(\beta_{m}^{1} \alpha_{1}^{1}\right)+\cdots+\delta_{1}^{m_{l_{1}}}\left(\beta_{m}^{1} \alpha_{1}^{m l_{l_{1}}}\right)+\cdots+\right.} \\
& \delta_{r}^{1}\left(\beta_{m}^{1} \alpha_{1}^{r}\right)+\cdots+\delta_{r}^{m l_{r}}\left(\beta_{m}^{1} \alpha_{r}^{m_{l r}}\right)+\cdots+ \\
& \left.\delta_{t}^{1}\left(\beta_{m}^{1} \alpha_{t}^{1}\right)+\cdots+\delta_{t}^{m_{l_{t}}}\left(\beta_{m}^{1} \alpha_{t}^{m_{l_{t}}}\right), \cdots\right] \\
& =\left[\sum_{s=1}^{t} \sum_{u_{s}=1}^{m_{l_{1}}} \delta_{s}^{u_{s}}\left(\beta_{m}^{1} \alpha_{s}^{u_{s}}\right), \cdots, \sum_{s=1}^{t} \sum_{u_{s}=1}^{m_{l_{s}}} \delta_{s}^{u_{s}}\left(\beta_{m}^{v_{j m}} \alpha_{s}^{u_{s}}\right)\right]
\end{aligned}
$$

Portanto, as relações de $\Gamma$ que começam em $\hat{i}_{n}$ e terminam em $\hat{j}_{m}$, são dadas por $\sum_{s=1}^{t} \sum_{u_{s}=1}^{m_{l_{s}}} \delta_{s}^{u_{s}}\left(\beta_{m}^{v_{m}} \alpha_{s}^{u_{s}}\right)=0$, para cada $v_{m}=1, \cdots, v_{j_{m}}$ e $m=1, \cdots, r$.

Observamos que as relações de $\Gamma$ são exatamente aquelas que começam no vértice $\hat{i}_{n}$, pois todos os outros vértices estão associados à projetivos cujos radicais são, também, projetivos (cf. Lema 3.2, Lema 3.3, e Lema 3.4). Ou seja, as relações de $\Gamma$ são definidas por somas de caminhos de comprimento dois, começando em $\hat{i}_{n}$ e terminando nos vértices $\hat{j}_{1}, \cdots, \hat{j}_{r}$, da maneira exposta acima.

Segue que $\Gamma \cong k Q /_{I}$, onde $Q$ é a aljava definida no parágrafo 3.3 , e $I$ é o ideal de $k Q$, gerado pelos caminhos $\sum_{s=1}^{t} \sum_{u_{s}=1}^{m_{l_{s}}} \delta_{s}^{u_{s}}\left(\beta_{m}^{v_{m}} \alpha_{s}^{u_{s}}\right)$, para cada $m=1, \cdots, r$ fixado.

Vejamos um desenho para fixar as idéias do resultado acima. Considere o seguinte esboço local de $Q(\Lambda)$ :

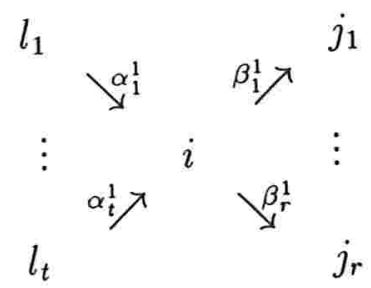


Então, temos que $f \circ \beta_{m}$ é morfismo em $\Gamma$ induzido pela multiplicação por $\beta_{m}^{1} . \alpha_{s}^{1}$, produto de flechas em $Q(\Lambda)$. Observamos que podem existir mais de uma flecha ligando os vértices, que não estão desenhadas para maior clareza de nossas idéias.

Observe o desenho a seguir, trata-se de um esboço do comportamento local de $Q(\Gamma)$ :

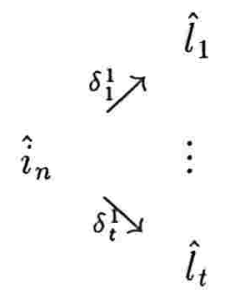

Como vimos acima, cada vértice $\hat{l}_{s}$ se liga ao vértice $\hat{j}_{m}$ através da flecha $\gamma_{l_{s}, j_{m}}^{u_{s}, v_{m}}$, definida pela $s$-ésima coordenada da $m$-ésima componente do morfismo $f_{*}$. Apresentamos abaixo um desenho, para fixarmos estas idéias

$$
\begin{array}{ccc}
\hat{l}_{1} & \stackrel{\left(\beta_{m}^{1} \alpha_{1}^{1}\right)}{\longrightarrow} & \hat{j}_{m} \\
\vdots & \left(\beta_{m}^{1} \alpha_{t}^{1}\right) \nearrow & \\
\hat{l}_{t} & &
\end{array}
$$

para cada $m=1, \ldots, r$.

Finalmente, podemos desenhar um esboço local de $Q(\Gamma)$, da seguinte maneira:

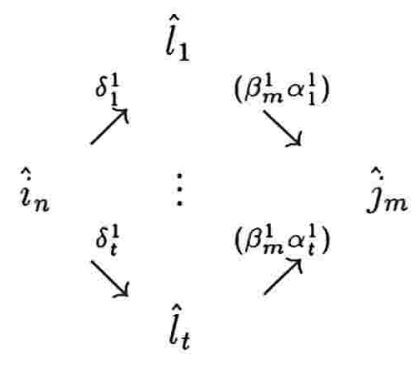

para cada $m=1, \ldots, r$.

\section{Exemplos:}


1. Seja $\Lambda=k Q$ com $Q$ dada por:

$$
\begin{array}{rl} 
& \\
& \uparrow \\
4 \\
& \uparrow \\
1 \rightarrow 2 \rightarrow 3 & 3 \leftarrow 5 \leftarrow 6
\end{array}
$$

e o $\Lambda$-módulo inclinante $T=\tau^{-} S_{3} \oplus \underset{j \neq 3}{\bigoplus} P_{j}$, associado ao vértice 3 . Então $\Gamma$ é dada por:

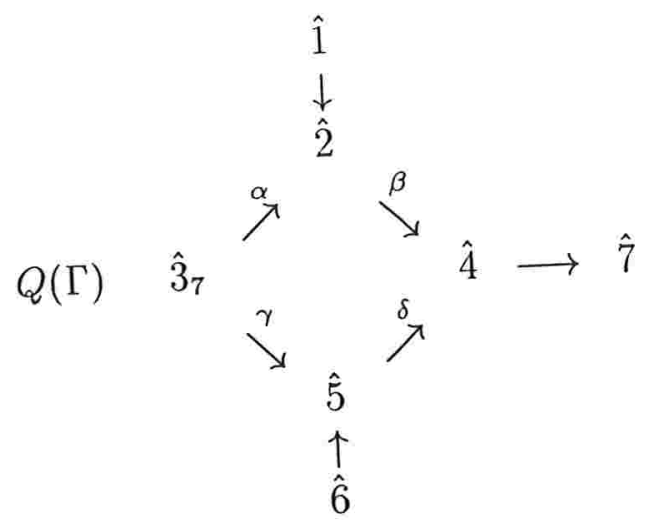

onde $\beta \alpha+\delta \gamma=0$.

2. Se $\Lambda$ é dada por:

$$
Q: 1^{\swarrow} \rightarrow^{\searrow^{\beta}} 3
$$

e $T=\tau^{-} S_{1} \oplus \underset{j \neq 1}{\bigoplus} P_{j}$ então $\Gamma$ será dada por:

$$
\hat{1}_{3} \stackrel{\bar{\delta}}{\longrightarrow} \hat{2} \underset{\bar{\beta}}{\stackrel{\bar{\alpha}}{\longrightarrow}} \hat{3}
$$

$\operatorname{com} \bar{\alpha} \bar{\delta}=0$, e $\bar{\beta}$ equivalente à $\beta$.

3. Seja $\Lambda$ dada por:

$$
1 \stackrel{\gamma}{\leftarrow} 2 \underset{\beta}{\stackrel{\alpha}{\leftarrow}} 3
$$


e T o módulo inclinante associado ao vértice 2 , ou seja, $T=\tau^{-} S_{2} \oplus$ $P_{1} \oplus P_{3}$.

Então, $\Gamma$ é dada por:

$$
\hat{2}_{3} \underset{\delta_{2}}{\stackrel{\delta_{1}}{\rightleftarrows}} \hat{3} \underset{\tilde{\beta}_{\gamma}}{\stackrel{\tilde{\alpha}_{\gamma}}{\rightrightarrows}} \hat{1}
$$

$\operatorname{com} \delta_{1} \tilde{\alpha}_{\gamma}+\delta_{2} \tilde{\beta}_{\gamma}=0$

\subsection{Os módulos de Koszul sobre as álgebras BB-inclinadas}

Neste parágrafo, estaremos considerando o $\Lambda$-módulo $B B$-inclinante $T=$ $\tau^{-} S_{i} \oplus \underset{j \neq i}{\bigoplus} P_{j}$, com $\Lambda$ hereditária e $\Gamma=\operatorname{End}_{\Lambda}(T)^{o p}$, como nos parágrafos anteriores. Lembremos que, no parágrafo anterior, provamos que $\Gamma$ é uma álgebra de Koszul.

É fato conhecido que, numa álgebra inclinada, todo módulo que pertença à classe $\mathcal{Y}(T)$, tem dimensão projetiva um. Como observamos antes, a classe de torção em $\Gamma$-mod é gerada pelo simples $\hat{S}=\hat{S}_{\hat{i}_{n}} \cong \operatorname{Ext}_{\Lambda}^{1}\left(T, S_{i}\right)$, que é um $\Gamma$-módulo de Koszul com dimensão projetiva dois, se $\Gamma$ não é hereditária. Assim, somente os módulos que possuam somandos diretos em add $\hat{S}$ terão dimensão projetiva 2 .

Os resultados a seguir relacionam, sob certas condições, os módulos de Koszul sobre $\Lambda$ e sobre $\Gamma$. Daqui por diante, estaremos considerando $\Gamma=\Gamma_{i}$, a álgebra Brenner-Butler inclinada associada ao vértice $i$, como definimos no início deste capítulo. Estaremos, também, considerando os $\Lambda$-módulos $M \in \mathcal{T}(T)$, indecomponíveis, e tais que $M \notin$ add $T$, pois quando $M \in \operatorname{add} T$ temos que $\operatorname{Hom}_{\Lambda}(T, M)$ é projetivo, e portanto, um $\Gamma$-módulo de Koszul.

Lema 3.14 Sejam $M \in \mathcal{T}(T)$ um $\Lambda$-módulo indecomponível, não projetivo, e $\mathcal{M}=\operatorname{Hom}_{\Lambda}(T, M)$, um $\Gamma=\Gamma_{i}$-módulo. Suponhamos que $\hat{S}$ não é somando direto de $\frac{\mathcal{M}}{r_{\Gamma} \mathcal{M}}$ e fixemos $\mathcal{P}:\left[0 \longrightarrow P_{(1)} \stackrel{f}{\longrightarrow} P_{(0)} \longrightarrow M \longrightarrow 0\right]$ a $\Lambda$-resolução projetiva minimal de $M$. Então, $\operatorname{Hom}_{\Lambda}(T,-)(\mathcal{P})$ é uma $\Gamma$-resolução projetiva minimal de $\mathcal{M}$. 
Demonstração: Aplicamos o funtor $\operatorname{Hom}_{\Lambda}(T,-)$ a resolução projetiva de $M$ acima, e obtemos a seqüência exata longa de $\Gamma$-módulos dada por:

$$
\begin{gathered}
0 \longrightarrow\left(T, P_{(1)}\right) \stackrel{f_{*}}{\longrightarrow}\left(T, P_{(0)}\right) \longrightarrow(T, M)= \\
=\mathcal{M} \stackrel{\delta}{\longrightarrow} \operatorname{Ext}_{\Lambda}^{1}\left(T, P_{(1)}\right) \longrightarrow \operatorname{Ext}_{\Lambda}^{1}\left(T, P_{(0)}\right) \longrightarrow \operatorname{Ext}_{\Lambda}^{1}(T, M) \longrightarrow 0
\end{gathered}
$$

Como $M \in \mathcal{T}(T)$, temos que $\operatorname{Ext}_{\Lambda}^{1}(T, M)=0$ e que $\frac{M}{r M} \in \mathcal{T}(T)$. Então $P_{(0)} \in \mathcal{T}(T)$ e, portanto, $P_{i}$ não é somando direto de $P_{(0)}$. Segue que $\operatorname{Ext}_{\Lambda}^{1}\left(T, P_{(0)}\right)=0$.

Como $\hat{S}$ não é somando direto do topo de $\mathcal{M}$, o morfismo $\delta$ é zero, pois $\operatorname{Ext}_{\Lambda}^{1}\left(T, P_{i}\right) \cong \operatorname{Ext}_{\Lambda}^{1}\left(T, S_{i}\right)=\hat{S}$.

Logo, $\operatorname{Ext}_{\Lambda}^{1}\left(T, P_{(1)}\right)=0$ e obtemos a $\Gamma$-resolução projetiva para $\mathcal{M}$ dada por:

$$
0 \longrightarrow\left(T, P_{(1)}\right) \longrightarrow\left(T, P_{(0)}\right) \longrightarrow \mathcal{M} \longrightarrow 0
$$

Pela $B B$-equivalência entre módulos de torção (cf. em $[\mathrm{AS} 1]$ ) segue que, ela é minimal, desde que $M \in \mathcal{T}(T)$.

Proposição 3.15 Nas mesmas hipóteses do Lema 3.14, assuma, também, que $M$ e $\mathcal{M}$ são ambos gerados em grau zero e que $P_{i}$ não é somando direto de $r_{\Lambda} P_{(0)}$. Então, $\mathcal{M}$ é um $\Gamma$-módulo de Koszul se e somente se $M$ é um $\Lambda$-módulo de Koszul.

Demonstração: Assuma que $\mathcal{M}$ é um $\Gamma$-módulo de Koszul. Então a resolução projetiva de $\mathcal{M}$, apresentada no lema anterior, é linear.

Segue que

$$
r_{\Gamma}\left(T, P_{(1)}\right)=r_{\Gamma}^{2}\left(T, P_{(0)}\right) \cap\left(T, P_{(1)}\right) .
$$

Pelo Lema 3.3, e como $P_{i}$ não é somando direto de $P_{(1)}$, podemos concluir que $r_{\Gamma}\left(T, P_{(1)}\right)=\left(T, r_{\Lambda} P_{(1)}\right)$. O mesmo argumento nos diz que $r_{\Gamma}^{2}\left(T, P_{(0)}\right)=$ $r_{\Gamma}\left(T, r_{\Lambda} P_{(0)}\right)$. Usando novamente que $P_{i}$ não é somando direto de $r_{\Lambda} P_{(0)}$, obtemos a seguinte igualdade $r_{\Gamma}^{2}\left(T, P_{(0)}\right)=\left(T, r_{\Lambda}^{2} P_{(0)}\right)$. Segue que $\left(T, r_{\Lambda} P_{(1)}\right)$ $=\left(T, r_{\Lambda}^{2} P_{(0)} \cap P_{(1)}\right)$.

Pela $B B$-equivalência entre módulos de torção, e como ambos são submódulos de $P_{(1)}$, podemos concluir que $r_{\Lambda} P_{(1)}=r_{\Lambda}^{2} P_{(0)} \cap P_{(1)}$.

Como $M$ é gerado em grau zero, podemos concluir que $M$ é um $\Lambda$-módulo de Koszul. 
Suponha, agora, que $M$ é um $\Lambda$-módulo de Koszul. Como $\Lambda$ é hereditária temos que $r_{\Lambda} M$ é um $\Lambda$-módulo projetivo (cf [G-M,2]), e portanto a seqüência exata curta induzida pela $\Lambda$-resolução projetiva de $M$, dada por:

$$
0 \longrightarrow P_{(1)} \longrightarrow r_{\Lambda} P_{(0)} \longrightarrow r_{\Lambda} M \longrightarrow 0 \text { cinde }
$$

Como $P_{i}$ não é somando direto de $r_{\Lambda} P_{(0)}$, temos que $\left(T, r_{\Lambda} P_{(0)}\right)$ é um $\Gamma$-módulo projetivo.

Aplicando o funtor $\operatorname{Hom}_{\Lambda}(T,-)$ à seqüência $\left({ }^{*}\right)$ obtemos que:

$$
0 \longrightarrow\left(T, P_{(1)}\right) \longrightarrow\left(T, r_{\Lambda} P_{(0)}\right) \longrightarrow\left(T, r_{\Lambda} M\right) \longrightarrow 0
$$

é seqüência exata curta, pois $\operatorname{Ext}_{\Lambda}^{1}\left(T, P_{(1)}\right)=0$. Logo, cinde. Portanto, temos que $\left(T, P_{(1)}\right)$ é um somando direto de $\left(T, r_{\Lambda} P_{(0)}\right)=r_{\Gamma}\left(T, P_{(0)}\right)$.

Como está suposto ser $\mathcal{M}$ gerado em grau zero, podemos concluir que $\mathcal{M}$ é um módulo de Koszul sobre $\Gamma$.

\section{Observações:}

1. Nem todo módulo de Koszul sobre $\Lambda$ produz um módulo de Koszul sobre $\Gamma$, como mostra o exemplo abaixo.

Sejam $\Lambda$ dada por:

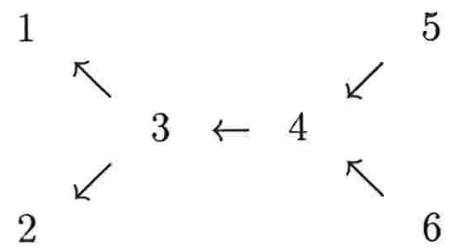

e $T$ o $\Lambda$-módulo inclinante associado ao vértice 1 . Notemos que $T$ é um APR-inclinante, e neste caso, temos que $\Gamma$ é hereditária.

Temos que $\operatorname{Hom}_{\Lambda}\left(T, S_{3}\right)$, cuja $k$-representação é dada por:

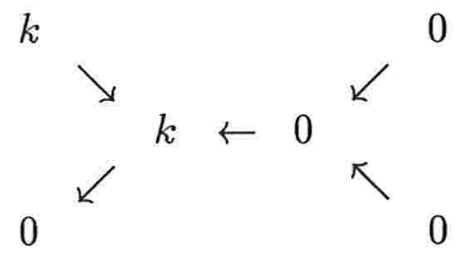

não é um módulo de Koszul, pois seu radical não é projetivo, porém $S_{3}$ é um $\Lambda$-módulo de Koszul. 
2. Nas condições da Proposição 3.15 temos que a hipótese " $P_{i}$ não é somando direto de $r_{\Lambda} P_{(0)}$ ", pode ser substituída por " $\hat{S}_{\hat{l}_{1}}, \cdots, \hat{S}_{\hat{l}_{t}}$ não são somandos diretos do topo de $\mathcal{M}^{\prime \prime}$.

Com efeito, se $P_{i}$ não é somando direto de $r_{\Lambda} P_{(0)}$ então $P_{l_{s}}$ não é somando direto de $P_{(0)}$, para todo $s=1, \cdots, t$. Ou equivalentemente, $\left(T, P_{l_{j}}\right)$ não é somando direto de $\left(T, P_{(0)}\right)$, para $s=1, \cdots, t$. Portanto, $\hat{S}_{\hat{l}_{s}}$ não é somando direto do topo de $\mathcal{M}$, para cada $s=1, \cdots, t$.

Reciprocamente, a conclusão anterior nos diz que $\left(T, P_{l_{s}}\right)$ não aparece como somando direto de $\left(T, P_{(0)}\right)$, para cada $s=1, \cdots, t$. O mesmo ocorre entre $P_{l_{s}}$ e $P_{(0)}$ e consequentemente entre $r_{\Lambda} P_{l_{s}}$ e $r_{\Lambda} P_{(0)}$. Logo, $P_{i}$ não é somando direto de $r_{\Lambda} P_{(0)}$.

Lema 3.16 Seja $\mathcal{M}=\operatorname{Hom}_{\Lambda}(T, M)$ um módulo sobre $\Gamma=\Gamma_{i}$, com $M \in$ $\mathcal{T}(T)$ indecomponível. Então, $\hat{S}$ é um somando direto de $\frac{\mathcal{M}}{r_{\Gamma} \mathcal{M}}$ se e somente se $P_{i}$ é um somando direto de $\Omega^{1}(M)$.

Demonstração: Observemos que $\operatorname{Ext}_{\Lambda}^{1}\left(T, P_{(0)}\right)=0$ e que é exata a seqüência longa dada por:

$$
0 \longrightarrow\left(T, P_{(1)}\right) \longrightarrow\left(T, P_{(0)}\right) \longrightarrow(T, M) \stackrel{\delta}{\longrightarrow} \operatorname{Ext}_{\Lambda}^{1}\left(T, P_{(1)}\right) \longrightarrow 0
$$

obtida da resolução projetiva minimal de $M$, apresentada no Lema 3.14.

Como $P_{(1)}$ é projetivo, teremos que $\operatorname{Ext}_{\Lambda}^{1}\left(T, P_{(1)}\right) \cong(\hat{S})^{m}$, para algum $m \in \mathbb{N}$. Portanto, podemos concluir que:

$P_{i}$ é somando direto de $P_{1} \Leftrightarrow m \neq 0 \Leftrightarrow \delta \neq 0 \Leftrightarrow \hat{S}$ é um somando direto do topo de $\mathcal{M}$.

$O$ resultado a seguir estende para álgebras $B B$-inclinadas, o fato apresentado na Prop.6.1 em [G-M,2], para o caso de módulos cuja cobertura projetiva não intercepta $\hat{P}_{\hat{i}_{n}}=P_{\Gamma}(\hat{S})$.

Proposição 3.17 Sejam $M \in \mathcal{T}(T)$ indecomponivel com $M \notin$ add $T$ e o $\Gamma=\Gamma_{i}$-módulo $\mathcal{M}=\operatorname{Hom}_{\Lambda}(T, M)$, ambos gerado em grau zero, e tal que $\hat{S}$ não é somando direto de $\frac{\mathcal{M}}{r_{\Gamma} \mathcal{M}}$. Então, $\mathcal{M}$ é $\Gamma$-módulo de Koszul se e somente se $r_{\Gamma} \mathcal{M}$ é projetivo. 
Demonstração: Se $r_{\Gamma} \mathcal{M}$ é projetivo, a Proposição 2.10, garante que $\mathcal{M}$ é módulo de Koszul.

Suponhamos que $\mathcal{M}$ seja um módulo de Koszul. Desde que $\hat{S}$ não é somando direto do topo de $\mathcal{M}$, podemos considerar a $\Gamma$-resolução projetiva

$$
0 \longrightarrow\left(T, P_{(1)}\right) \stackrel{f_{\bullet}}{\longrightarrow}\left(T, P_{(0)}\right) \longrightarrow(T, M) \longrightarrow 0
$$

obtida no Lema 3.14 .

Do fato de $\mathcal{M}$ ser Koszul, resulta que $\left(T, P_{(1)}\right)$ é um somando direto da cobertura projetiva de $r_{\Gamma}\left(T, P_{(0)}\right)$. Conforme o Lema 3.5 , temos que $r_{\Gamma}\left(T, P_{(0)}\right)$ é um $\Gamma$-módulo projetivo. Logo, o monomorfismo $\left(T, P_{(1)}\right) \hookrightarrow$ $r_{\Gamma}\left(T, P_{(0)}\right)$, induzido por $f_{*}$, cinde. Assim, a seqüência exata curta

$$
0 \longrightarrow\left(T, P_{(1)}\right) \longrightarrow r_{\Gamma}\left(T, P_{(0)}\right) \longrightarrow r_{\Gamma}(T, M) \longrightarrow 0,
$$

induzida pela $\Gamma$-resolução projetiva de $\mathcal{M}$, cinde.

Segue que $r_{\Gamma}(T, M)$ é um $\Gamma$-módulo projetivo.

Os próximos resultados nos dizem quais são os $\Lambda$-módulos $M$, tal que $(T, M)$ tem topo interceptando os simples $\hat{S}=\hat{S}_{\hat{i}_{n}}, \hat{S}_{\hat{l}_{1}}, \cdots, \hat{S}_{\hat{l}_{t}}$, que são $\Gamma$ módulos de Koszul.

Proposição 3.18 Seja $M$ o $\Lambda$-módulo definido por

$$
M=\operatorname{coker}\left(P_{j_{m}} \stackrel{g_{m, s}}{\longrightarrow} P_{l_{s}}\right)
$$

onde $g_{m, s}: P_{j_{m}} \longrightarrow P_{l_{s}}$ é a aplicação induzida por um caminho de comprimento 2 que liga os vértices $j_{m}$, $i$ e $l_{s}$ para $m=1, \cdots, r$ e $s=1, \cdots, t$, fixados. Suponhamos que $\mathcal{M}=\operatorname{Hom}_{\Lambda}(T, M)$ seja um módulo graduado sobre $\Gamma=\Gamma_{i}$, gerado em grau zero. Então, $\mathcal{M}$ é Koszul.

Demonstração: Temos que $M \in \mathcal{T}(T)$ e que a seqüência abaixo é exata:

$$
\begin{gathered}
0 \longrightarrow P_{j_{m}} \stackrel{g_{m, s}}{\longrightarrow} P_{l_{s}} \longrightarrow \underset{\|}{\operatorname{conuc}} g_{m, s} \longrightarrow 0 \\
M
\end{gathered}
$$

Aplicando o funtor $\operatorname{Hom}_{\Lambda}(T,-)$ a esta seqüência, obtemos a seguinte seqüência exata curta de $\Gamma$-módulos:

$$
0 \longrightarrow\left(T, P_{j_{m}}\right) \stackrel{g_{m, s}^{*}}{\longrightarrow}\left(T, P_{l_{s}}\right) \longrightarrow \mathcal{M} \longrightarrow 0
$$


Pelo Lema 3.4 , e a resolução projetiva minimal do simples $\hat{S}_{\hat{l}_{s}}$, é fácil mostrar que a $\Gamma$-resolução projetiva acima, é linear. Logo, $\mathcal{M}$ é um $\Gamma$-módulo de Koszul.

Proposição 3.19 Sejam $M \in \mathcal{T}(T)$ um $\Lambda$-módulo graduado e gerado em grau zero, e o $\Gamma=\Gamma_{i}$-módulo $\mathcal{M}=\operatorname{Hom}_{\Lambda}(T, M)$. Suponhamos que $\mathcal{M}$ seja graduado e gerado em grau zero e tal que sua cobertura projetiva é o projetivo $\hat{P} \in \operatorname{add} \hat{P}_{\hat{i}_{n}}$. Se $\mathcal{M}$ é Koszul então $M$ é Koszul.

Demonstração: Seja a $\Lambda$-resolução projetiva minimal de $M$, dada por:

$$
0 \longrightarrow P_{(1)}(M) \stackrel{f}{\longrightarrow} P_{M} \stackrel{\pi}{\longrightarrow} M \longrightarrow 0
$$

Queremos mostrar que esta é uma resolução linear.

Observamos que $\mathcal{M} \notin$ add $\hat{S}$, pois $\mathcal{M} \in \mathcal{Y}(T)$. Assim, podemos considerar a $\Gamma$-resolução projetiva de $\mathcal{M}$

$$
0 \longrightarrow(T, P) \longrightarrow\left(T, \coprod_{m} \tau^{-} S_{i}\right) \stackrel{p^{*}}{\longrightarrow} \mathcal{M} \longrightarrow 0
$$

com $P$ projetivo em add $T, m>0$ e $p_{*}$ induzido por $p: \underset{m}{\amalg} \tau^{-} S_{i} \longrightarrow M$ um $\Lambda$ morfismo, que existe, pela BB-equivalência entre os módulos de torção. Como $M \in \mathcal{T}(T)$, segue que $\operatorname{Ext}_{\Lambda}^{1}(T, M)=0$, e portanto, temos pelas fórmulas de Auslander que $(M, S)=0$. Resulta disso, que $P_{M} \in$ add $T$, e, consequentemente, que $\left(T, P_{M}\right)$ é um $\Gamma$-módulo projetivo. Assim, o $\Gamma$-morfismo induzido por $\pi$ se fatora através de $p_{*}$. Mais claramente, podemos considerar o diagrama comutativo dado por:

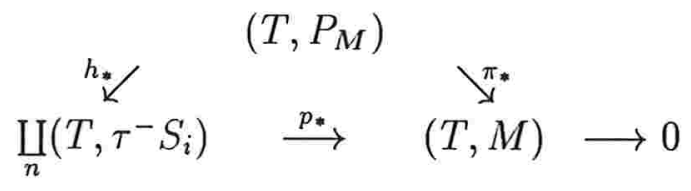

onde $\pi_{*}=p_{*} \circ h_{*}$.

Note que $\operatorname{Im} h_{*} \subseteq r_{\Gamma}\left(T, \coprod_{m} \tau^{-} S_{i}\right)$.

Agora, aplique o funtor $\operatorname{Hom}_{\Lambda}(T,-)$ à seqüência $\left({ }^{*}\right)$ para obtermos a seguinte seqüência exata longa:

$$
0 \longrightarrow\left(T, P_{(1)}(M)\right) \longrightarrow\left(T, P_{M}\right) \stackrel{\pi_{*}}{\longrightarrow}(T, M) \longrightarrow \operatorname{Ext}_{\Lambda}^{1}\left(T, P_{(1)}(M)\right) \longrightarrow 0
$$


com $\operatorname{Ext}_{\Lambda}^{1}\left(T, P_{(1)}(M)\right) \neq 0$, pois $\left.P_{(1)}(M)\right) \notin \mathcal{T}(T)$, pelo Lema 3.16.

Temos que $p_{*}\left(r_{\Gamma}\left(T, \coprod_{n} \tau^{-} S_{i}\right)\right)=r_{\Gamma}(T, M) \neq 0$, pois $\mathcal{M}$ não é semi-simples.

Além disso, $\hat{S} \notin \operatorname{supp} \frac{r_{\Gamma}(T, M)}{r_{\Gamma}^{2}(T, M)}$, pois $\Gamma$ não tem circuitos orientados. Segue que a seqüência abaixo é exata:

$$
0 \longrightarrow\left(T, P_{(1)}(M)\right) \stackrel{f_{*}}{\longrightarrow}\left(T, P_{M}\right) \stackrel{\pi_{*}}{\longrightarrow} r_{\Gamma}(T, M) \longrightarrow 0
$$

Portanto, esta seqüência é uma $\Gamma$-resolução projetiva de $r_{\Gamma}(T, M)$. Mas, $\left(^{*}\right)$ é minimal, então $\operatorname{Im} f \subseteq r_{\Lambda} P_{M}$. Como $r_{\Gamma}\left(T, P_{M}\right)=\left(T, r_{\Lambda} P_{M}\right)$, por argumentos análogos aos usados nos Lemas 3.2 e 3.4 , segue que $\operatorname{Im} f_{*} \subseteq$ $r_{\Gamma}\left(T, P_{M}\right)$. Ou seja, $\left({ }^{* *}\right)$ é minimal.

Sabemos que sobre uma álgebra de Koszul, o radical de um módulo de Koszul é um módulo de Koszul (cf. [GM, 1]). Segue que (**) é uma resolução linear. Ou seja, $\left(T, P_{(1)}\right)$ é um somando direto da cobertura projetiva de $r_{\Gamma}\left(T, P_{M}\right)$.

Mas, $r_{\Gamma}^{2} \mathcal{M}$ é módulo projetivo, pois $\hat{S}$ não é somando direto do topo $r \mathcal{M}$ (veja proposição 3.17 ); portanto, a seqüência exata curta abaixo, induzida por $(* *)$, cinde:

$$
0 \longrightarrow\left(T, P_{(1)}(M)\right) \longrightarrow r_{\Gamma}\left(T, P_{M}\right) \longrightarrow r_{\Gamma}^{2}(T, M) \longrightarrow 0
$$

Segue, então, que $\left(T, P_{(1)}(M)\right)$ é um somando direto de $r_{\Gamma}\left(T, P_{M}\right)$.

Observamos que $P_{M}=\bigoplus_{s} P_{l_{s}}^{n_{s}} \bigoplus \underset{j \neq l_{s}}{\bigoplus_{j}} P_{\text {l }}$ e portanto $r_{\Lambda} P_{M}=$ $\bigoplus_{s}\left(P_{i}^{m_{s}} \oplus P_{l_{s}}^{\prime}\right)^{n_{s}} \bigoplus_{j \neq l_{s}} r P_{j}$. Mas, vimos acima, que $\operatorname{Ext}_{\Lambda}^{1}\left(T, P_{(1)}\right) \neq 0$. Assim, podemos concluir que $P_{(1)}(M)$ é um somando direto de $r_{\Lambda} P_{M}$ e com isso provamos que $M$ é um $\Lambda$-módulo de Koszul.

Existem vários exemplos de álgebras de caminhos $\Lambda$, que tem sua classe de módulos de Koszul, $\mathcal{K}(\Lambda)$, finita, mas tal que $\mathcal{K}(\Gamma)$ é infinita. E viceversa. Por isso, se tornou interessante responder que tipo de módulos sobre $\Lambda$, induzem, pelo funtor $\operatorname{Hom}_{\Lambda}(T,-)$, módulos de Koszul sobre $\Gamma$.

Observamos que os resultados apresentados nas proposições 3.16 e 3.20 nos dizem que os módulos de Koszul sobre $\Gamma$, com cobertura projetiva dada por add $\hat{P}$, onde $\hat{P}=\hat{P}_{\hat{i}_{n}} \oplus \underset{r}{\oplus} \hat{P}_{\hat{r}}$ com $r \notin\left\{l_{1}, \cdots, l_{t}\right\}$, são definidos por módulos de Koszul em $\Lambda$.

Mostramos, em seguida, que os $\Gamma$-módulos $\operatorname{Hom}_{\Lambda}\left(T\right.$, conuc $\left[P_{j_{m}} \stackrel{g_{m, s}}{\longrightarrow}\right.$ $\left.P_{l_{s}}\right]$ ), onde $g_{m, s}$ é o morfismo definido pela multiplicação por caminhos, como 
na Proposição 3.19 , tem cobertura projetiva dada por cópias de $\hat{P}_{\hat{l}_{s}}$, para cada $m=1, \cdots, r$ e $s=1, \cdots, t$, fixados e podem determinar uma classe infinita de módulos de Koszul.

Ou seja, a classe de módulos $\mathcal{K}(\Gamma)$ pode ser infinita, e para que isso ocorra, não é necessário que a classe de módulos $\mathcal{K}(\Lambda)$ também seja, mas, bastaria que a classe dos módulos cuja apresentação é dada como definimos acima, seja infinita.

No caso $\Gamma=\Gamma_{i}$, vimos pela observação 2 , (enunciada em seguida a Proposição 3.15), que se $\mathcal{M}=\operatorname{Hom}_{\Lambda}(T, M)$ é um $\Gamma$-módulo tal que supp $\frac{\mathcal{M}}{r \mathcal{M}}$ não intercepta o conjunto dado pelos simples $\left\{\hat{S}, \hat{S}_{\hat{l}_{1}}, \cdots, \hat{S}_{\hat{l}_{t}}\right\}$, então temos que Mé Koszul $\Leftrightarrow M$ é Koszul.

Provamos, também, que os módulos $\mathcal{M}$ com topo em add $\hat{S}$ são produzidos pelos módulos de Koszul $M$ na categoria $\Lambda$-mod, tal que $P_{i}$ é um somando direto de $\Omega^{1}(M)$.

Com estes resultados, conseguimos entender a relação existente entre as finitudes das classe $\mathcal{K}(\Lambda)$ e $\mathcal{K}(\Gamma)$, e também, obtivemos um critério para determinar se $\mathcal{K}(\Gamma)$ é infinita.

Finalmente, do fato de que todo módulo de dimensão projetiva um com apresentação linear é um módulo de Koszul e que o único simples de dimensão projetiva dois de $\Gamma_{i}$ é, também, um módulo de Koszul, que gera a classe dos módulos de torção, podemos concluir que, sobre as álgebras BB-inclinadas, $\mathcal{L}(\Gamma)=\mathcal{K}(\Gamma)$.

Nossos próximos resultados nos mostram como se comportam os submódulos e quocientes de módulos de Koszul, sobre as álgebras $B B$-inclinadas. Resultados nesta direção e de caráter mais geral, foram obtidos e apresentados no Capítulo 2.

Proposição 3.20 Sejam $M \in \mathcal{T}(T)$ e o $\Gamma=\Gamma_{i}$-módulo $\mathcal{M}=\operatorname{Hom}_{\Lambda}(T, M)$, tal que $\mathcal{M}$ é um $\Gamma$-módulo de Koszul. Sejam $P_{\Gamma}(\mathcal{M})=\underset{\hat{j} \neq \hat{i}_{n}}{\amalg} \hat{P}_{\hat{j}} \amalg\left(\hat{P}_{\hat{i}_{n}}\right)^{m} a$ cobertura projetiva de $\mathcal{M}$ e $\mathcal{M}^{\prime}$ submódulo graduado de $\mathcal{M}$ dado pela imagem homomórfica de $\underset{\hat{j} \neq \hat{i}_{n}}{\hat{P}_{\hat{j}}}$, sobre a cobertura projetiva de $\mathcal{M}$ e tal que $p d_{\Gamma} \mathcal{M}^{\prime}=$ 1 e $\frac{\mathcal{M}}{\mathcal{M}^{\prime}}$ não é um $\Gamma$-módulo projetivo. Então, $\mathcal{M}^{\prime}$ é um $\Gamma$-módulo de Koszul se e somente se $\hat{S}_{\hat{i}_{n}}$ não é um somando direto de $\frac{\mathcal{M}}{\mathcal{M}^{\prime}}$.

Demonstração: Temos que a inclusão $\mathcal{M}^{\prime} \stackrel{j}{\longrightarrow} \mathcal{M}$ induz o monomorfismo que 
cinde $\frac{\mathcal{M}^{\prime}}{r \mathcal{M}^{\prime}} \stackrel{\bar{j}}{\longrightarrow} \frac{\mathcal{M}}{r \mathcal{M}}$. Pela Proposição 2.13, temos que $\mathcal{M}^{\prime}$ é um módulo de Koszul se e somente se, $\operatorname{pd}_{\Gamma} \frac{\mathcal{M}}{\mathcal{M}^{\prime}}=1$. E é claro que $\operatorname{pd}_{\Gamma} \frac{\mathcal{M}}{\mathcal{M}^{\prime}}=1$ se e somente se $\hat{S}_{\hat{i}_{n}}$ não é somando direto de $\frac{\mathcal{M}}{\mathcal{M}^{\prime}}$.

Proposição 3.21 Considere $\Gamma_{i}$ e o módulo $\mathcal{M}$ como na proposição 3.20.

Seja $\mathcal{M}^{\prime \prime}$ submódulo graduado de $\mathcal{M}$ tal que $\mathcal{M}^{\prime \prime}$ é a imagem homomorfica de $\coprod_{n} \hat{P}_{i_{n}}$, através da cobertura projetiva de $\mathcal{M} e \frac{\mathcal{M}}{\mathcal{M}^{\prime \prime}}$ não é um módulo projetivo. Então $\mathcal{M}^{\prime \prime}$ é Koszul.

Demonstração: Se $\operatorname{pd}_{\Gamma} \mathcal{M}^{\prime \prime}=0$, então $\mathcal{M}^{\prime \prime}$ é um módulo de Koszul. Se $\operatorname{pd}_{\Gamma} \mathcal{M}^{\prime \prime} \neq 0$, então temos que $\operatorname{pd}_{\Gamma} \mathcal{M}^{\prime \prime}=1$, pois $\mathcal{M}^{\prime \prime} \subset \mathcal{M} \in \mathcal{Y}(T)$. Como $\hat{S}_{\hat{i}_{n}}$ não é somando direto de $\frac{\frac{\mathcal{M}}{\mathcal{M}^{\prime \prime}}}{r_{\Gamma}\left(\frac{\mathcal{M}}{\mathcal{M}^{\prime \prime}}\right)}$, podemos concluir que $\operatorname{pd}_{\Gamma} \frac{\mathcal{M}}{\mathcal{M}^{\prime \prime}}=1$, e pela Proposição 2.13 segue que $\mathcal{M}^{\prime \prime}$ é um $\Gamma$-módulo de Koszul.

O exemplo a seguir, nos mostra que pode ocorrer que $\frac{\mathcal{M}}{\mathcal{M}^{\prime}}$ e $\mathcal{M}$ sejam módulos de Koszul, mas $\mathcal{M}^{\prime}$ não ser um módulo de Koszul. Seja $\Gamma$ a álgebra $B B$-inclinada dada por:

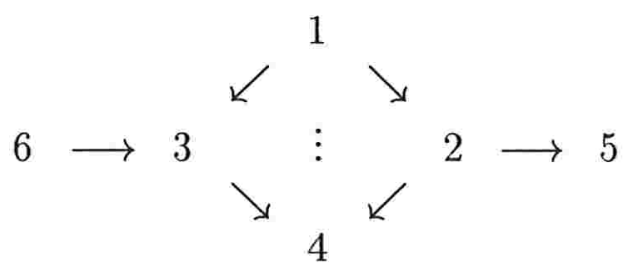

Considere o $\Gamma$-módulo $\mathcal{M}$ cuja $k$-representação é dada por:

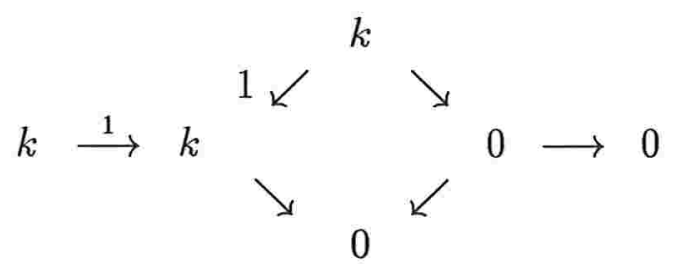

Temos que $\mathcal{M}$ tem $\Gamma$-resolução projetiva dada por:

$$
0 \longrightarrow P_{3} \oplus P_{2} \longrightarrow P_{1} \oplus P_{6} \longrightarrow \mathcal{M} \longrightarrow 0
$$


Assim, $\mathcal{M}$ é Koszul. Seja $\mathcal{M}^{\prime}$ submódulo de $\mathcal{M}$ cuja $k$-representação é dada por:

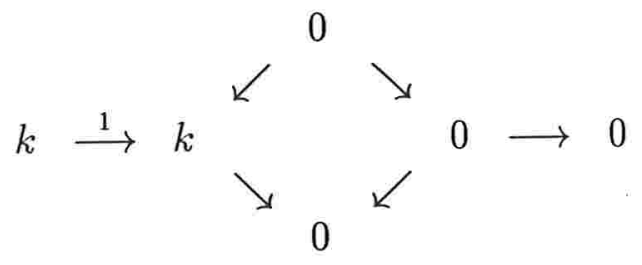

Temos que $\frac{\mathcal{M}}{\mathcal{M}^{\prime}} \cong S_{1}$ é um módulo de Koszul, mas $\mathcal{M}^{\prime}$ não, já que tem $\Gamma$-resolução projetiva dada por:

$$
0 \longrightarrow P_{4} \longrightarrow P_{6} \longrightarrow \mathcal{M}^{\prime} \longrightarrow 0
$$

com $P_{4}$ somando direto de $r^{2} P_{6}$. Observamos que $\operatorname{pd}_{\Gamma} S_{1}=2$.

\subsection{Uma generalização para as álgebras $B B-$ inclinadas}

Neste parágrafo, apresentamos um resultado que nos permitiu identificar um outro exemplo de álgebras de Koszul inclinadas.

Estamos mantendo aqui a notação que temos utilizado até aqui.

Consideremos $\theta$ o conjunto de índices $\{1,2, \ldots, u\} \subset Q_{0}$ tais que $m_{1}, \ldots$, $m_{u}$ são vértices na aljava de $\Lambda$ tomados de forma que não existem arestas no grafo da aljava ligando $m_{s}$ a $m_{j}$ para $s, j=1, \cdots, u$.

Seja $T_{\theta}$ o $\Lambda$-módulo dado por

$$
T_{\theta}=\tau^{-} S_{m_{1}} \oplus \cdots \oplus \tau^{-} S_{m_{u}} \oplus \bigoplus_{\substack{j \in Q_{0} \\ j \notin \theta}} P_{j}
$$

Temos que $T_{\theta}$ é um $\Lambda$-módulo inclinante. Com efeito, é claro que pd $T_{\theta} \leq$ 1, e também, que $\operatorname{Ext}_{\Lambda}^{1}\left(T_{\theta}, T_{\theta}\right)=0$, pois, para cada $j$ e cada $s$ em $\{1, \ldots, u\}$, distintos, é fato que $\operatorname{Ext}_{\Lambda}^{1}\left(\tau^{-} S_{m_{j}}, \tau^{-} S_{m_{s}}\right)=\operatorname{DHom}_{\Lambda}\left(\tau^{-} S_{m_{j}}, S_{m_{s}}\right)=$ $\operatorname{Ext}_{\Lambda}^{1}\left(S_{m_{j}}, S_{m_{s}}\right)=0$. Além disso, o número de somandos de $T_{\theta}$ é o número $\Lambda$-módulos simples não isomorfos.

Nosso objetivo, nesta seção, é mostrar que se $\Gamma_{\theta}=\operatorname{End}_{\Lambda}\left(T_{\theta}\right)^{o p}$ é uma álgebra graduada cuja apresentação é dada por um ideal graduado, então é 
uma álgebra de Koszul. Para isso, precisaremos demonstrar o resultado a seguir.

Considere sobre a álgebra hereditária $\Lambda$, os módulos projetivos indecomponíveis $P$ e $Q$, com $S_{p}$ e $S_{q}$ os simples do topo destes projetivos, associados aos vértices $p$ e $q$, respectivamente, tal que estes vértices não estejam ligados por arestas em $Q(\Lambda)$.

Proposição 3.22 Sejam $p$ e $q$, definidos acima e seja $T=\tau^{-} S_{p} \oplus \tau^{-} S_{q} \oplus$, $\bigoplus_{\substack{j \in Q_{0} \\ j \neq p, q}} P_{j}$ tal que $\Gamma_{0}=\operatorname{End}_{\Lambda}(T)^{o p}$, o anel de endomorfismo de $T$ sobre $\Lambda$, é uma álgebra graduada com alguma apresentação dada por um ideal graduado. Então, $\Gamma_{0}$ é uma álgebra de Koszul.

Demonstração: Sabemos que a classe dos módulos livre de torção de $\Lambda$-mod é dada por $\mathcal{F}=$ Cogen $(\tau T)$. Como $\tau T=S_{p} \oplus S_{q}$, segue que $\mathcal{X}(T)=$ Gen $\left(\operatorname{Ext}^{1}\left(T, S_{p} \oplus S_{q}\right)\right)$. Observando que, para todo $S$

$$
\operatorname{Ext}_{\Lambda}^{1}(T, S) \cong D \operatorname{Hom}_{\Lambda}(S, \tau T)=D \operatorname{Hom}_{\Lambda}\left(S, S_{p} \oplus S_{q}\right)
$$

segue que $\operatorname{Ext}_{\Lambda}^{1}\left(T, S_{p}\right)$ e $\operatorname{Ext}_{\Lambda}^{1}\left(T, S_{q}\right)$ são $\Gamma$-módulos simples e estão em $\mathcal{X}(T)$.

Vamos denota-los por $\hat{S}_{\hat{p}}$ e $\hat{S}_{\hat{q}}$, respectivamente. Queremos mostrar que ambos são módulos de Koszul. É suficiente verificar para o caso em que tenham dimensão projetiva dois, pois, todo módulo simples de dimensão projetiva um, sobre uma álgebra graduada é um módulo de Koszul.

Sem perda de generalidade, podemos assumir que $\operatorname{Hom}_{\Lambda}(P, Q)=0$. Como os vértices $p$ e $q$ não estão ligados por arestas, podemos visualizar a situação local na aljava de $\Lambda$, relativamente a estes vértices, da seguinte forma:

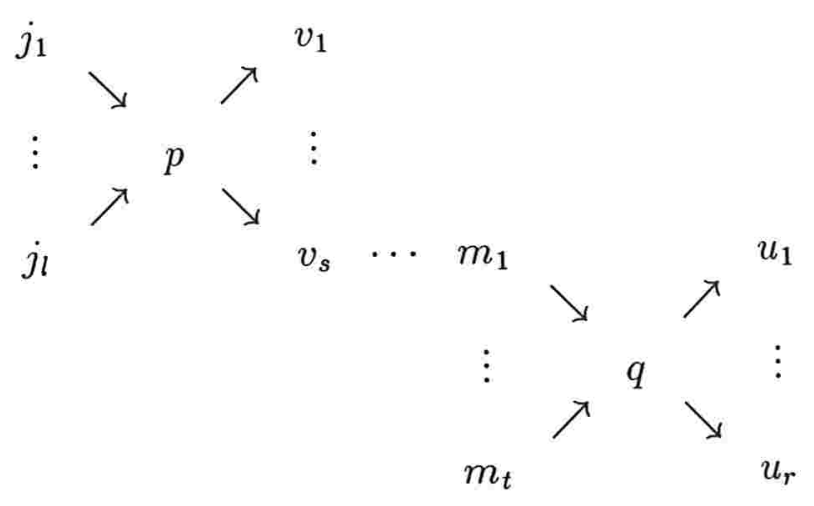


Observamos que podemos ter mais de uma flecha ligando os vértices, e que foram suprimidas no desenho.

Como foi feito na Proposição 3.8, considerando a gr $\Lambda$-resolução projetiva de $\tau^{-} S_{p}$ dada por:

$$
0 \longrightarrow P \longrightarrow P_{j_{1}}^{m_{1}} \oplus \cdots \oplus P_{j_{u}}^{m_{u}} \stackrel{\pi}{\longrightarrow} \tau^{-} S_{p} \longrightarrow 0
$$

analogamente ao que obtivemos no Teorema 3.6, onde $m_{l}$ é o número de flechas de $p$ para $j_{l}$, na aljava de $\Lambda, \operatorname{com} l=1, \cdots, u$, obtemos que

$$
0 \longrightarrow\left(T, r_{\Lambda} P\right) \longrightarrow \bigoplus_{l=1}^{u}\left(T, P_{j_{l}}\right)^{m_{l}} \stackrel{\pi_{*}}{\longrightarrow}\left(T, \tau^{-} S_{p}\right) \longrightarrow \operatorname{Ext}_{\Lambda}^{1}(T, P) \cong \hat{S}_{\hat{p}} \longrightarrow 0 .
$$

Pelas condições 1 e 2, equivalentes a definição de módulo de Koszul, de acordo com [GM,1], e que foram apresentadas na seção 1.1, basta mostrar que $r_{\Gamma}\left(T, r_{\Lambda} P\right)=r_{\Gamma}^{2}\left(T, \bigoplus_{l} P_{j_{l}}^{m_{l}}\right) \cap\left(T, r_{\Lambda} P\right)$, pois $\Gamma_{0}$ é uma álgebra graduada.

Claramente, temos que $\left(T, r_{\Lambda} P\right) \subset r_{\Gamma}\left(T, \bigoplus_{l} P_{j_{l}}^{m_{l}}\right)$, pois a resolução acima é minimal. Como $\tau^{-} S_{p}$ é um $\Lambda$-módulo de Koszul, temos que $r_{\Lambda} P=r_{\Lambda}^{2}\left(\bigoplus_{l} P_{j_{l}}^{m_{l}}\right)$ $\cap P$, e portanto, temos que $\left(T, r_{\Lambda} P\right) \subset\left(T, \bigoplus_{l} r_{\Lambda}^{2} P_{j_{l}}^{m_{l}}\right)$.

Seja $P_{j_{l}}^{\prime} \in$ add $T$ módulo projetivo tal que $r P_{j_{l}}=P^{m_{l}} \oplus P_{j_{l}}^{\prime}$, para cada $l=$ $1, \cdots, u$ e $m_{l}$, como definimos acima. Então, temos que $r^{2} P_{j_{l}}=r P^{m_{l}} \oplus r P_{j_{l}}^{\prime}$ e, como $P \notin \mathcal{T}(T)$, segue que $r\left(T, P_{j_{l}}\right)=\left(T, r P^{m_{l}} \oplus P_{j_{l}}^{\prime}\right)$. Assim, obtemos que

$$
\begin{aligned}
r_{\Gamma}^{2}\left(T, \oplus_{l=1}^{u} P_{j_{l}}^{m_{l}}\right) & =\oplus_{l=1}^{u}\left(T,\left(r P^{m_{l}} \oplus P_{j_{l}}^{\prime}\right)^{m_{l}}\right) \\
=\left(T, \oplus_{l=1}^{u}\left(r^{2} P^{m_{l}} \oplus r P_{j_{l}}^{\prime}\right)^{m_{l}}\right) . & =\oplus_{l=1}^{u}\left(T,\left(r^{2} P^{m_{l}} \oplus r P_{j_{l}}^{\prime}\right)^{m_{l}}\right)
\end{aligned}
$$

Portanto, temos que $r_{\Gamma}^{2}\left(T, \oplus_{l} P_{j_{l}}^{m_{l}}\right) \cap\left(T, r_{\Lambda} P\right)=\left(T,\left[\oplus_{l=1}^{u}\left(r^{2} P^{m_{l}} \oplus r P_{j_{l}}^{\prime}\right)^{m_{l}} \cap\right.\right.$ $r P)$.

Mas, $r P=\oplus_{l=1}^{u}\left(r P^{m_{l}} \oplus r P_{j_{l}}^{\prime}\right)^{m_{l}} \cap P=r P \oplus\left[\oplus_{l=1}^{u}\left(r P_{j_{l}}^{\prime}\right)^{m_{l}} \cap P\right]$, nos diz que a interseção $\oplus_{l=1}^{u}\left(r P_{j_{l}}^{\prime}\right)^{m_{l}} \cap P$ é zero. Como temos que $\oplus_{l=1}^{u}\left(r^{2} P^{m_{l}} \oplus\right.$ $\left.r P_{j_{l}}^{\prime}\right)^{m_{l}} \cap r P=r^{2} P \oplus\left[\oplus_{l=1}^{u}\left(r P_{j_{l}}^{\prime}\right)^{m_{l}} \cap r P\right]$, segue que o termo entre parênteses, nesta última soma, é zero.

Assim, obtemos que $\left(T, r_{\Lambda}^{2} P\right)=r_{\Gamma}^{2}\left(T, \oplus_{l} P_{j_{l}}^{m_{l}}\right) \cap\left(T, r_{\Lambda} P\right)$. Como $r(T, r P) \cong$ $\left(T, r^{2} P\right)$, segue o que queriamos mostrar. Portanto,$\hat{S}_{\hat{p}}$ é um $\Gamma$-módulo de Koszul. 
Para mostrar que $\hat{S}_{Q}$ é um $\Gamma$-módulo de Koszul usamos os mesmos argumentos apresentados acima, para o caso so simples $\hat{S}_{\hat{P}}$. A situação é análoga, pois, se considerarmos a gr $\Lambda$-resolução projetiva de $\tau^{-} S_{q}$ dada por:

$$
0 \longrightarrow Q \stackrel{g}{\longrightarrow} \bigoplus_{y=1}^{t} Q_{v_{y}}^{m_{y}} \stackrel{\phi}{\longrightarrow} \tau^{-} S_{Q} \longrightarrow 0
$$

onde $m_{y}$ é o número de flechas do vértice $v_{y}$ para o vértice $q$, obtemos que

$$
0 \longrightarrow\left(T, r_{\Lambda} Q\right) \longrightarrow\left(T, \bigoplus_{y} Q_{v_{y}}\right)^{m_{y}} \longrightarrow\left(T, \tau^{-} S_{Q}\right) \longrightarrow \hat{S}_{\hat{q}} \longrightarrow 0
$$

é $\Gamma$-resolução projetiva linear de $\hat{S}_{Q}$. Com efeito, $\bigoplus_{y} Q_{v_{y}}^{m_{y}}$, os somandos diretos de $\bigoplus_{y} r_{\Lambda} Q_{v_{y}}$ distintos de $Q$ e $r_{\Lambda} Q$ são todos módulos em add $T$. Também, temos que $\tau^{-} S_{q}$ é módulo de Koszul e, portanto, vale que $r_{\Lambda} Q=r_{\Lambda}^{2}\left(\oplus_{y} Q_{v_{y}}^{m_{y}}\right) \cap$ $Q$. Um cálculo rápido mostra que $r_{\Gamma}\left(T, r_{\Lambda} Q\right)=r_{\Gamma}^{2}\left(T, \underset{y}{\oplus} Q_{v_{y}}^{m_{y}}\right) \cap\left(T, r_{\Lambda} Q\right)$.

Ou seja, $\hat{S}_{\dot{q}}$ é um $\Gamma$-módulo de Koszul.

Como todo módulo simples sobre $\Gamma_{0}$ é um módulo de Koszul, segue que esta é uma álgebra de Koszul.

Observemos que $\Gamma_{0} \cong k Q / I$, onde $Q$ é uma aljava finita e $I$ é um ideal admissível graduado de $k Q$, por hipótese. Vimos que os únicos módulos simples sobre $\Gamma$, que podem ter dimensão projetiva 2 , são os descritos acima, que denotamos por $\hat{S}_{\hat{p}}$ e $\hat{S}_{\hat{q}}$. Assim, podemos considerar que o ideal das relações de $\Gamma$ é gerado por combinação lineares de caminhos que começam em $p$ e $q$, (cf em [B]). Pelo que mostramos acima, podemos concluir que estes caminhos são quadráticos. Segue que $\Gamma_{0}$ é álgebra graduada quadrática.

Agora, podemos repetir a argumentação que usamos para mostrar que $\hat{S}_{\hat{p}}$ é módulo de Koszul e concluir que cada $\Gamma_{\theta}$-módulo simples $\hat{S}_{\hat{m}_{j}}$, com $j=1, \cdots, u$, e $\theta$ o conjunto de índices definido no início deste parágrafo, é um módulo de Koszul e o resultado que queremos segue como conseqüência.

Teorema 3.23 Seja $\Lambda$ uma k-álgebra hereditária de dimensão finita. Seja $T=\bigoplus_{j=1}^{t} \tau^{-} S_{l_{j}} \oplus \underset{m \neq l_{j}}{\bigoplus_{m}} P_{m}$ um $\Lambda$-módulo inclinante tal que $\Gamma=\operatorname{End}_{\Lambda}(T)^{o p} e^{\prime}$ 
uma álgebra graduada com alguma apresentação dada por um ideal graduado. Então $\Gamma$ é álgebra de Koszul.

Demonstração: Temos que cada $\Gamma$-módulo $\operatorname{Ext}_{\Lambda}^{1}\left(T, S_{m_{j}}\right)$ é um $\Gamma$-módulo simples de torção. Pela Proposição 3.22, estes módulos são $\Gamma$-módulos de Koszul, segue que $\Gamma$ é Koszul. 


\section{Capítulo 4}

\section{Álgebras inclinadas graduadas}

Neste capítulo, estaremos apresentando alguns estudos e resultados importantes, sobre álgebras inclinadas graduadas, sendo o mais importantes destes, o que permite decidir quando estas álgebras são álgebras de Koszul.

\subsection{Introdução}

Estaremos considerando $\Lambda$, uma álgebra de dimensão finita sobre o corpo algebricamente fechado $k$, e $T$ um módulo inclinante sobre $\Lambda$, graduado e gerado em grau zero. Consideraremos $\Gamma=\operatorname{End}_{\Lambda}(T)^{o p}$, o anel de endomorfismos de $T$ sobre $\Lambda$.

Fixada uma $\mathbb{Z}$-graduação para uma álgebra de Artin $\Gamma$, sabemos que os módulos simples e os projetivos são graduáveis, o radical e o socle de um módulo graduavel é homogêneo e, em particular, o radical de uma álgebra de Artin graduada é um ideal homogêno graduado. A verificação destes fatos pode ser vista, por exemplo, na Proposicão 3.5, em [G-G]. Também, neste mesmo trabalho, Green e Gordon, mostram que $\Gamma$ é uma álgebra de Artin graduada, com uma $\mathbb{Z}$-graduação induzida pelo grau dos morfismos homogêneos de $T$ em $T$. Por outro lado, se ocorrer que $\Gamma$ é o quociente de uma álgebra de caminhos, digamos $\Gamma=k Q / I$, onde $Q$ é uma aljava finita e $I$ é um ideal admissível, graduado, podemos considerar sobre $\Gamma$, uma outra graduação, a saber, a graduação induzida pelo comprimento das flechas. Estas duas $\mathbb{Z}$-graduações sobre $\Gamma$, serão detalhadas nas seções 4.2 e 4.3 . 
Observamos que as afirmações apresentadas em [GG], podem ser generalizadas para as álgebras de Artin $G$-graduadas, onde $G$ é um grupo livre de torção, como foi provado em [FGGM]; também neste artigo, os autores mostraram que existem álgebras locais, não semi-simples, que não tem nenhuma graduação não trivial; em [GHM], podem ser encontradas mais informações sobre esta questão.

Ainda no parágrafo 4.3 , mostramos, através de exemplos, que não é possível relacionar as graduações sobre $\Gamma$, que mencionamos acima, através de funtores graduados. No parágrafo 4.4, apresentaremos resultados que são conseqüências de condições impostas sobre a componente de grau zero de $\Gamma$, no caso de ser $\Gamma$ uma álgebra inclinada.

Em seguida, apresentaremos um estudo detalhado da resolução projetiva de $\Gamma / r$, onde $r$ é o radical de Jacobson de $\Gamma$, e esta álgebra é dada pelo anel de endomorfismo de um módulo inclinante, sobre uma álgebra hereditária de dimensão finita, ou seja, $\Gamma$ é uma álgebra inclinada.

A definição que obtivemos, para os morfismos que definem a cobertura projetiva do radical de projetivos indecomponíveis de $\Gamma$, se aplica ao caso em que $\Lambda$ é $k$-álgebra de dimensão finita. Sua principal conseqüência foi motivar a caracterização dos morfismos que possuem a propriedade de serem elementos de $r$ que não pertencem a $r^{2}$, e, por outro lado, sob a ótica do Teorema de Gabriel, os morfismos que são induzidos pela multiplicação por flechas da aljava de $\Gamma$.

Em seguida, no parágrafo 4.7, introduzimos a noção de morfismo poço de torção, que se originou da idéia de restringir o conceito de morfismo quase cindido à classe dos módulos de torção de $\Lambda$. Sabemos que este conceito está definido sobre álgebras de Artin, assim, apesar de inicialmente estarmos interessados em álgebras inclinadas, pudemos obter resultados para o anel de endomorfismo de um módulo inclinante, sobre uma $k$-álgebra de dimensão finita, não necessariamente hereditária. No caso de $\Lambda$ ser hereditária, temos a vantagem de conhecer a sua aljava de $A R$, mais detalhadamente, o que nos permite identificar gráficamente, os predecessores de um somando direto indecomponível do módulo inclinante, assim como os domínios e contra-domínios possiveis dos morfismos, e em particular, os morfismos quase cindidos. Assim, nossas aplicações e exemplos se voltaram, com mais ênfase, para a classe das álgebras inclinadas.

No parágrafo 4.8, apresentamos nosso principal resultado, que caracteriza 
as álgebras inclinadas graduadas Koszul, e alguns exemplos e aplicações, como conseqüência deste resultado.

No parágrafo 4.9 apresentamos exemplos que ilustram nossos resultados, incluindo o caso das álgebras inclinadas generalizadas de tipo $A_{n}$. Mais tres exemplos, foram apresentados no parágrafo 4.10 , e ilustram a relação entre as classes $\mathcal{L}(\Gamma)$ e $\mathcal{K}(\Gamma)$.

\subsection{Graduações sobre álgebras de caminhos}

Neste seção, vamos descrever a graduação que pode ser induzida sobre uma álgebra de caminhos, através de suas flechas.

Consideremos $\Lambda$ uma $k$-álgebra de dimensão finita. Construímos o anel graduado de $\Lambda$ associado a $I$, um ideal próprio de $\Lambda$, que denotaremos por $\operatorname{Gr}_{\Lambda}(I)$, da maneira descrita a seguir.

Tomemos a seqüência decrescente de ideais dada por:

$$
I^{0}=\Lambda \supset I^{1}=I \supset I^{2} \supset I^{3} \supset \cdots \supset I^{n} \supset \cdots
$$

e consideremos os quocientes $A^{(n)}=\frac{I^{n}}{I^{n+1}}$. É claro que $I^{(n)} \cdot I^{(m)} \subset I^{(n+m)}$. Portanto, dados dois elementos homogêneos $x \in I^{(n)}$ e $y \in I^{(m)}$, podemos definir uma multiplicação $\bar{x} \cdot \bar{y}=\overline{x \cdot y}+I^{(n+m+1)}$, onde $x \in I^{(n)}$ e $y \in I^{(m)}$, que define uma estrutura de anel para a soma direta de todos os $A^{(n)}$, com $n \geq 0$.

Resulta disso, que o anel $\operatorname{Gr}_{\Lambda}(I)=\coprod_{n \geq 0} A^{(n)}$ é um anel graduado, chamado de anel graduado de $\Lambda$ associado a $I$. Observamos que esta construção pode ser feita no caso mais geral, em que $\Lambda$ é uma álgebra de Artin.

Um importante caso particular pode ser obtido ao tomarmos $I$ o radical de $\Lambda$. Neste caso, temos que

$$
\operatorname{Gr}_{\Lambda}(\operatorname{rad} \Lambda)=\Lambda^{(0)} \oplus \Lambda^{(1)} \oplus \cdots
$$

onde $\Lambda^{(n)}=\frac{\operatorname{rad}^{n} \Lambda}{\operatorname{rad}^{n+1} \Lambda}$, para $n \geq 0$. Se $\Lambda$ for uma $k$-álgebra graduada, de decomposição básica, 1-gerada, então $\Lambda$ e $\operatorname{Gr}_{\Lambda}(\operatorname{rad} \Lambda)$, são isomorfas como álgebras graduadas; (cf. em [GM,1]). 
É fato conhecido que o quociente de uma álgebra de caminhos por um ideal admissível graduado é uma $k$-álgebra graduada, de decomposição básica, 1-gerada. Segue que o anel graduado desta álgebra associado ao seu radical graduado, nos permitirá entender esta álgebra, como uma álgebra graduada com a graduação dada pelas flechas. Se a álgebra não for 1-gerada, não é verdade que ela e o anel graduado associado ao seu radical sejam isomorfos como álgebras graduadas, como mostra o exemplo 3, abaixo.

A seguir ilustramos esta graduação com alguns exemplos.

Exemplo 1: Seja $\Gamma$ a $k$-álgebra dada por:

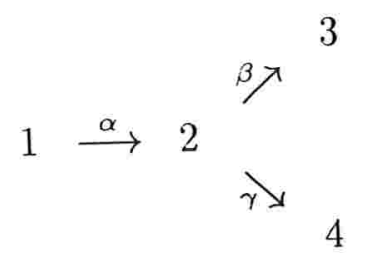

com $\gamma \alpha=0=\beta \alpha$.

Então, temos que $\Gamma^{(0)}$ é o $k$-espaço vetorial gerado pelos idempotentes, $e_{1}, e_{2}, e_{3}, e_{4}$, associados aos vértices de $Q(\Gamma) ; \Gamma^{(1)}$ é o $k$-espaço vetorial gerado pelas flechas $\alpha, \beta$ e $\gamma ; \Gamma^{(2)} \cong\left(\Gamma^{(1)}\right)^{2}$, e $\Gamma^{(n)}=0$, para cada $n>2$.

Exemplo 2: Seja $\Gamma$ a $k$-álgebra dada por:

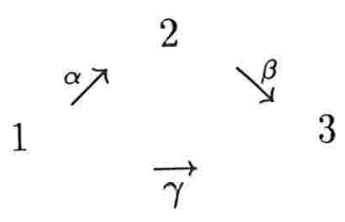

Temos que $\Gamma^{(0)}$, a componente de grau zero de $\Gamma$ é o $k$-espaço vetorial gerado pelos idempotentes $e_{1}, e_{2}, e_{3}$ e $\Gamma^{(1)}$, a componente de grau um, gerada por $\alpha, \beta, \gamma$. Temos, também, que $\Gamma^{(2)}=\left(\Gamma^{(1)}\right)^{2}$, e as componentes de grau maior que 2 são todas nulas.

Exemplo 3: Seja $\Gamma$ a álgebra dada por:

2

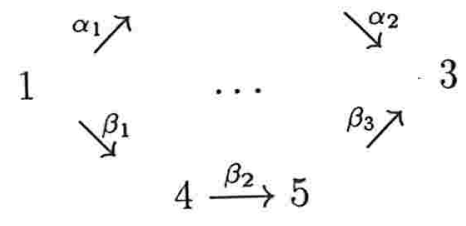


com a relação de comutatividade dada por $\alpha_{2} \alpha_{1}=\beta_{3} \beta_{2} \beta_{1}$.

Temos que $I$, o ideal das relações de $\Gamma$, não é homogêneo, e o anel graduado associado ao radical de $\Gamma$ não é isomorfo à $\Gamma$. Notemos que $P_{3}$ é gerado em grau 2, na graduação dada pelo radical de $\Gamma$, e em grau 3 na graduação dada pelo ideal $I$. No entanto, temos que $\Gamma^{(3)}=0$, em vista da relação de comutatividade definida sobre esta álgebra.

\subsection{A graduação induzida por morfismos ho- mogêneos}

Nesta seção, vamos descrever a graduação induzida pelo grau dos morfismos homogêneos entre módulos graduados indecomponíveis. Em particular, apresentaremos exemplos no caso em que tomamos a álgebra definida por um anel de endomorfismo de um módulo inclinante.

Seja $\Lambda$ uma $k$-álgebra de dimensão finita, graduada, 1-gerada, e de decomposição básica, ou seja, $\Lambda=k Q / I$, onde $Q$ é aljava finita e $I$ é ideal admissível graduado. Assim, podemos considerar $\Lambda$ como $k$-álgebra graduada, com a graduação induzida pelas flechas, mais precisamente, podemos considerar sobre $\Lambda$ a graduação definida pelo anel graduado associado ao radical de Jacobson graduado de $\Lambda$.

Tomemos $T$ um $\Lambda$-módulo graduado, gerado em grau zero. Digamos que seja $T=\bigoplus_{j=1}^{n} T_{j}$, onde cada somando direto $T_{j}$ é um $\Lambda$-módulo indecomponível, graduado pela graduação definida sobre $\Lambda$, que é dada por $T_{j}=\coprod_{m \geq 0} T_{j}^{(m)}$, onde $T_{j}^{(m)}=\Lambda^{(m)} \cdot T_{j}^{(0)}$ é a componente de grau $m$ do módulo $T_{j}$. Sem perda de generalidade, podemos assumir que $T$ é livre de multiplicidade, isto é, que os somandos diretos de indecomponíveis de $T$ não são isomorfos dois a dois.

A graduação induzida pelos morfismos homogêneos foi descrita em detalhes, no trabalho de green e Gordon, em [G-G], do qual obtivemos várias informações, que mencionaremos neste parágrafo. Antes disso, mostraremos com um exemplo, as graduações que podemos obter sobre os módulos, como as que descrevemos acima. 
Exemplo : Seja $\Lambda$ a álgebra de caminhos dada por:

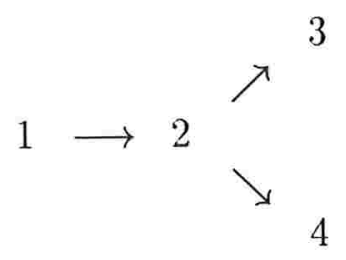

Consideremos sobre $\Lambda$, o módulo inclinante $T=\tau^{-} S_{2} \oplus P_{1} \oplus P_{2} \oplus P_{4}$. Temos que

$$
P_{j}=\Lambda e_{j} \cong \coprod_{m \geq 0} \Lambda^{(m)} e_{j}=\coprod_{m \geq 0} \frac{r^{m}}{r^{m+1}} e_{j}
$$

onde $e_{j}$ é idempotente de $\Lambda, j=1,3,4, r=\operatorname{rad} \Lambda$ e o isomorfismo é de módulos graduados. Também, temos que $\tau^{-} S_{2}=S_{1} \cong \Lambda^{(0)} \cdot e_{1}$.

De acordo com [GG], o $m$-translado de $T_{i}$ é o $\Lambda$-módulo dado por:

$$
\sigma(-m) T_{i}=\coprod_{s \in \mathbb{Z}} \bar{T}_{i}^{(s)} \text { onde } \bar{T}_{i}^{(s)}=T_{i}^{(s+m)} .
$$

Consideremos, agora, a decomposição de $\Gamma=\operatorname{End}_{\Lambda}(T)$, o anel de endomorfismo do $\Lambda$-módulo inclinante $T$, acima definido, dada por:

$$
\Gamma=\bigoplus_{j=1}^{n} \operatorname{Hom}_{\Lambda}\left(T, T_{j}\right)
$$

Seja $F_{\Lambda}:(\operatorname{gr} \Lambda)-\bmod \longrightarrow \Lambda-\bmod$, o funtor esquecimento. Para cada $j=1, \ldots, n$, consideraremos, como é feito em [GG], $\operatorname{Hom}_{\Lambda}\left(T, T_{j}\right)=$ $\operatorname{Hom}_{\Lambda}\left(F_{\Lambda} T, F_{\Lambda} T_{j}\right)$, e para cada $m \in \mathbb{Z}$, e cada $j=1, \cdots, n, \operatorname{Hom}_{\Lambda}\left(T, T_{j}\right)^{(m)}=$ Homgr $\Lambda\left(T, \sigma(-m) T_{j}\right)$, que é o grupo dos morfismos graduados de $T$ em $\sigma(-m) T_{j}$. Pelo Lema 2.1, em [GG], verifica-se que

$$
\operatorname{Hom}_{\Lambda}\left(T, T_{j}\right)=\coprod_{m \in \mathbb{Z}} F_{\Lambda}\left[\operatorname{Homgr} \Lambda\left(T, \sigma(-m) T_{j}\right)\right] .
$$

Como estamos assumindo que $T$ é gerado em grau zero, segue que $m \geq 0$. Por fim, se definirmos $g_{j}$ o comprimento graduado de $T_{j}$, para cada $j=$ $1, \ldots, n$, como sendo o maior inteiro $m$, não-negativo, tal que $T_{j}^{(m)} \neq 0$ mas $T_{j}^{(m+s)}=0$, para $s \geq m+1$, obtemos a seguinte expressão 


$$
\Gamma=\bigoplus_{j=1}^{n} \operatorname{Hom}_{\Lambda}\left(T, T_{j}\right)=\bigoplus_{j=1}^{n} \bigoplus_{m=0}^{g_{j}} F\left[\operatorname{Homgr} \Lambda\left(T, \sigma(-m) T_{j}\right)\right]
$$

Definidas graduações sobre as álgebras $\Lambda$ e $\Gamma$, podemos considerar a categoria dos objetos graduados de $\Lambda$-mod e $\Gamma$-mod que denotamos por $\operatorname{gr}(\Lambda)-\bmod$ e gr $(\Gamma)$-mod, respectivamente, cujos morfismos entre os objetos são os morfismos graduados.

Denotando $F_{\Lambda}$ e $F_{\Gamma}$ os funtores esquecimentos das categorias gr $(\Lambda)-\bmod$ e $\operatorname{gr}(\Gamma)-\bmod$ em $\Lambda$-mod e $\Gamma$-mod, respectivamente, obteremos o seguinte diagrama comutativo:

$$
\begin{array}{ccc}
\operatorname{gr}(\Lambda)-\bmod & \operatorname{Hom}_{\Lambda}(T,-) & \\
F_{\Lambda} \downarrow & & \operatorname{gr}(\Gamma)-\bmod \\
\Lambda-\bmod & \longrightarrow F_{\Gamma} \\
& & \Gamma-\bmod
\end{array}
$$

No entanto, pode ocorrer que, as duas graduações distintas que definimos sobre $\Gamma$, não estarem relacionadas por funtores graduados, como nos mostram os exemplos a seguir.

Exemplo 1: Consideremos $\Lambda$, a álgebra de caminhos do exemplo apresentado na seção anterior e tomemos o módulo inclinante $T=\tau^{-} S_{2} \oplus P_{1} \oplus P_{3} \oplus P_{4}$. Sabemos, pelos nossos resultados no capítulo 3 , que $\Gamma=\operatorname{End}_{\Lambda}(T)$ é a álgebra dada por:

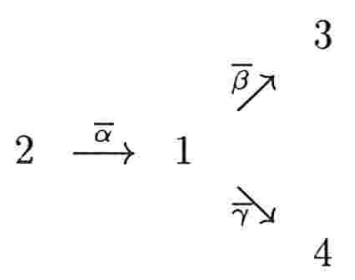

$\operatorname{com} \bar{\beta} \bar{\alpha}=0=\overline{\gamma \alpha}$.

Sobre $\Gamma$, temos a graduação-morfismo dada por:

$\Gamma^{(0)}=k \varepsilon_{1} \oplus k \varepsilon_{2} \oplus k \varepsilon_{3} \oplus k \varepsilon_{4} \oplus k \pi, \quad \Gamma^{(1)}=0, \quad \Gamma^{(2)}=k \cdot \beta \alpha \oplus k \cdot \gamma \alpha$, е $\Gamma^{(n)}=0$, para $n>2$, onde $\varepsilon_{j}: T \longrightarrow T_{j}$ é o morfismo dado por $\left(0, \ldots, 1_{T_{j}}, 0, \ldots\right)$, e as 
composições $\beta \alpha$ e $\gamma \alpha$ são os morfismos de $P_{3}$ e $P_{4}$ para $P_{1}$, respectivamente, e $\pi: P_{1} \longrightarrow \tau^{-} S_{2}=S_{1}$ é a cobertura projetiva de $S_{1}$ em $\Lambda$-mod.

Pelos resultados que obtivemos no capítulo 3 , podemos concluir que as morfismos entre os somandos diretos indecomponíveis de $T$, que induzem as flechas na aljava de $\Gamma$, tem graus zero e dois. Observamos que $\Gamma^{(0)}$ não é um módulo semi-simples no sentido usual, isto é, gerado por idempotentes ortogonais primitivos, e que $\Gamma$ não é uma álgebra hereditária. Também, convém observar que a morfismo $\pi$ é um epimorfismo de grau zero.

Exemplo 2: Sejam $\Lambda$ a $k$-álgebra dada por:

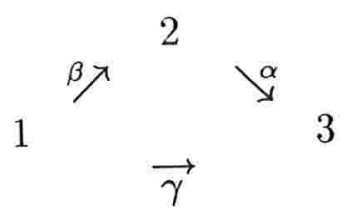

e $T=S_{1} \oplus \tau^{-} S_{3} \oplus P_{1}$ um módulo inclinante sobre $\Lambda$. Notemos que $\tau T=$ $\tau S_{1} \oplus S_{3}$. Temos que $\Gamma=\operatorname{End}_{\Lambda}(T)$ é a álgebra hereditária dada por:

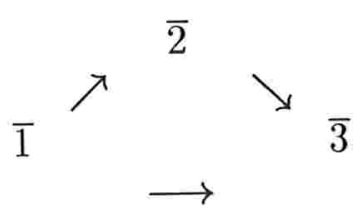

Pela graduação induzida pelo grau dos morfismos homogêneos entre somandos diretos de $T$, temos que $\Gamma$ é gerada em grau zero, pelos morfismos $\varepsilon_{\overline{1}}, \varepsilon_{\overline{2}}, \varepsilon_{\overline{3}}, \pi_{1}, \pi_{2}, p$, definidos por:

$\varepsilon_{\bar{j}}: T \longrightarrow T_{j}$, dada por $\varepsilon_{\bar{j}}=\left(0, \ldots, I_{T_{j}}, \ldots 0\right)$, onde $I_{T_{j}}$ é a função identidade sobre $T_{j} ; \pi_{1}: P_{1} \longrightarrow S_{1}$, a $\Lambda$-cobertura projetiva de $S_{1}, \pi_{2}$ : $P_{1} \longrightarrow \tau^{-} S_{3}$, uma componente da cobertura projetiva de $\tau^{-} S_{3}$, e $p$ : $\tau^{-} S_{3} \longrightarrow S_{1}$, um epimorfismo de $\tau^{-} S_{3}$ sobre o simples $S_{1}$.

Notemos que $\pi_{1}, \pi_{2}$ e $p$ são os morfismos que induzem morfismos entre os projetivos associados aos vértices $\overline{1}$ e $\overline{3}, \overline{2}$ e $\overline{3}, \overline{1}$ e $\overline{2}$, respectivamente. Como temos que $T$ é gerado em grau zero, segue que os morfismos de grau zero, entre somandos de $T$, somente podem ser fatorados por outros morfismos de grau zero. 
Exemplo 3: Tome $\Lambda$ a $k$-álgebra dada por:

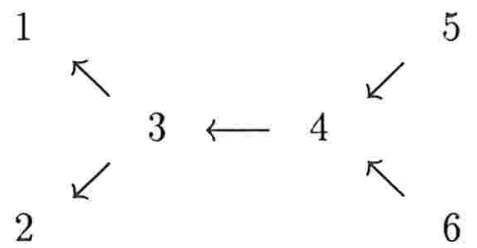

e seja $T=\tau^{-2} S_{1} \oplus \tau^{-} S_{2} \oplus P_{4} \oplus P_{1} \oplus P_{5} \oplus P_{6}$ um $\Lambda$-módulo inclinante. Notemos que $T$ é um módulo preprojetivo. Temos que $\Gamma=\operatorname{End}_{\Lambda}(T)$ é dada por:

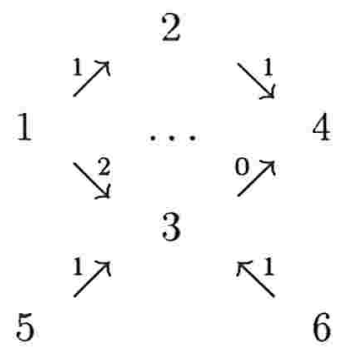

onde os números sobre as flechas indicam os menores graus possíveis, para os morfismos homogêneos entre os somandos diretos de $T$, que definem os projetivos associados aos vértices ligados por estas flechas.

\subsection{A apresentação projetiva minimal de $\Gamma / r$}

Neste parágrafo, estaremos interessados em identificar o tipo de aplicações homogêneas entre somandos diretos indecomponíveis do módulo inclinante, que aparecem nas resoluções projetivas dos módulos simples de álgebras inclinadas.

Assim, estaremos considerando $\Lambda$ uma $k$-álgebra de caminhos de dimensão finita, $T=\oplus_{j=1}^{n} T_{j}$ um $\Lambda$-módulo graduado inclinante, livre de multiplicidade, gerado em grau zero, e $\Gamma=\operatorname{End}_{\Lambda}(T)^{o p}$, o anel de endomorfismo de $T$ sobre $\Lambda$, uma álgebra inclinada, com a graduação apresentada em 4.3.

Seja $S_{l}$ um $\Gamma$-módulo simples, não projetivo, associado ao vértice $l$, cuja apresentação projetiva minimal sobre $\operatorname{gr}(\Gamma)$-mod é dada por:

$$
\bigoplus_{v=1}^{s} P_{j_{v}}^{m_{j_{v}}} \stackrel{f_{*}}{\longrightarrow} P_{l} \longrightarrow S_{l} \longrightarrow 0
$$


onde $f_{*}=\left(f_{*}, \ldots, f_{*}\right)$ é definida por $f_{v_{v}}(-)=f_{v}$ o- tal que $f_{v}: T_{j_{v}}^{m_{j_{v}}} \longrightarrow T_{l}$ é um $\Lambda$-morfismo que induz $f_{v}$ e $m_{j_{v}}$ é o número de vezes que $S_{j_{v}}$ aparece no topo de $r P_{l}$.

Como $S_{l}$ é um módulo simples, podemos assumir que $f_{*}$ é um morfismo graduado homogêneo. Como $T$ é gerado em grau zero, temos que grau de $f_{*}$ é não-negativo.

Se tomarmos o morfismo $\varepsilon_{v}: T \longrightarrow T_{j_{v}}$ com $v \in\{1, \ldots, s\}$ fixado, definido por $\varepsilon_{v}=\left(0, \ldots, I_{T_{j_{v}}}, 0, \ldots\right)$ e considerarmos a restrição de $f_{v}$ ao indecomponível $P_{j_{v}}$, que denotaremos por $f_{v}^{m_{v}}$, para cada $m_{v}=1, \cdots, m_{j_{v}}$, obteremos que

$$
f_{v}^{m_{v}}\left(\varepsilon_{v}\right)\left(T^{(0)}\right)=\left(f_{v}^{m_{v}} \circ \varepsilon_{v}\right)\left(T^{(0)}\right)=f_{v}^{m_{v}}\left(T_{j_{v}}^{(0)}\right)
$$

Segue que $f_{v}^{m_{v}}$ o $\varepsilon_{v}$ é um $\Lambda$-morfismo graduado homogêneo, com grau igual ao de $f_{v}^{m_{v}}$, que sabemos ser não-negativo pois $T$ é gerado em grau zero.

Proposição 4.1 Nas condições e notações usadas acima temos que $f_{*}$ é induzida ou por um monomorfismo ou por um epimorfismo.

Demonstração: Suponha que seja $f_{*}$ induzida por um $\Lambda$-morfismo $f$ tal que $f$ não é epimorfismo. Ou seja, temos que o conúcleo de $f$, que denotaremos por conuc $f$, é não nulo. Considere a seqüência exata curta de $\Lambda$-módulos dada por $0 \longrightarrow \operatorname{Im} f \stackrel{i}{\longrightarrow} T_{l} \longrightarrow$ conuc $f \longrightarrow 0$, onde $i$ é a inclusão canônica.

Como a classe dos módulos de torção é fechada por imagens, temos que Im $f \in \mathcal{T}(T)$; assim ao aplicarmos o funtor $\operatorname{Hom}_{\Lambda}(T,-)$ à seqüência acima, vamos obter a seqüência exata curta de $\Gamma$-módulos dada por:

$$
0 \longrightarrow \operatorname{Hom}_{\Lambda}(T, \operatorname{Im} f) \stackrel{i_{*}}{\longrightarrow} P_{l} \longrightarrow \operatorname{Hom}(T, \text { conuc } f) \longrightarrow 0
$$

pois $\operatorname{Ext}_{\Lambda}^{1}(T, \operatorname{Im} f)=0$. Considere, agora, o diagrama comutativo de $\Lambda$ módulos, dado por:

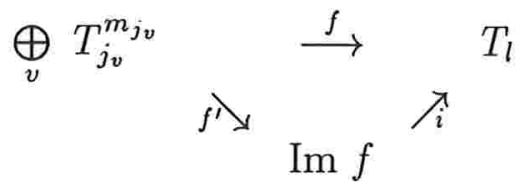

onde $f=i \circ f^{\prime}$. Então temos que $f_{*}=i_{*} \circ f_{*}^{\prime}$. 
Temos que $r_{\Gamma} P_{l} \cong \operatorname{Im} f_{*}=\operatorname{Im}\left(i_{*} \circ f_{*}^{\prime}\right)$; e como $i_{*}$ é monomorfismo, pois $i$ é monomorfismo e o funtor $\operatorname{Hom}_{\Lambda}(T,-)$ preserva monomorfismos, segue que $\operatorname{Im} f^{\prime} * \cong \operatorname{Im} f_{*} \cong r_{\Gamma} P_{l}$. Mais claramente, a equivalência de Brenner-Butler nos fornece um diagrama comutativo de $\Gamma$-módulos equivalente ao descrito acima, dado por:

$$
\begin{aligned}
& \bigoplus_{v} P_{j_{v}}^{m_{j_{v}}}=\bigoplus_{v} \operatorname{Hom}_{\Lambda}\left(T, T_{j_{v}}^{m_{j_{v}}}\right) \quad \stackrel{f_{*}}{\longrightarrow} \quad P_{l}=\operatorname{Hom}_{\Lambda}\left(T, T_{l}\right) \\
& f_{*}^{\lambda}(T, \operatorname{Im} f)^{\nearrow i_{*}}
\end{aligned}
$$

e, portanto, $(T, \operatorname{Im} f) \cong r_{\Gamma} P_{l}$. Segue daí que $S_{l} \cong(T$, conuc $f)$. Como conuc $f \in \mathcal{T}(T)$, temos que $S_{l} \in \mathcal{Y}(T)$ e, portanto, $\operatorname{pd}_{\Gamma} S_{l}=1$. Logo, $r_{\Gamma} P_{l}$ é um $\Gamma$-módulo projetivo dado por:

$$
r_{\Gamma} P_{l} \cong \bigoplus_{v} P_{j_{v}}^{m_{j_{v}}} \quad \text { e } \quad \operatorname{Im} f \cong \operatorname{Im} f^{\prime}=\bigoplus_{v} T_{j_{v}}^{m_{j_{v}}} .
$$

Segue que $f=i \circ f^{\prime}$ é um monomorfismo.

Como conseqüência desta proposição, temos o seguinte corolário:

Corolário 4.2 Com as notações anteriores, temos:

(i) $S_{l} \in \mathcal{Y}(T)$ se, e somente se, $f$ é um monomorfismo. Neste caso, temos que $S_{l} \cong \operatorname{Hom}_{\Lambda}(T$, conucf $)$, com conucf $\in \mathcal{T}(T)$.

(ii) $S_{l} \in \mathcal{X}(T)$ se, e somente se, $f$ é um epimorfismo. Neste caso, $S_{l} \cong$ Ext $t_{\Lambda}^{1}(T, n u c f)$, onde nucf $\in \mathcal{F}(T)$, se $p d S_{l}=1$, e nucf $\notin \mathcal{F}(T)$ se $p d S_{l}=2$.

Demonstração:

(i)Se $S_{l} \in \mathcal{Y}(T)$, então $\operatorname{pd} S_{l}=1$ e $S_{l} \cong \operatorname{Hom}_{\Lambda}(T, N)$, para algum $N \in \mathcal{T}(T)$. Segue que $f_{*}$ é um monomorfismo. Consideremos a seqüência exata curta $0 \longrightarrow\left(T, \oplus_{v} T_{j_{v}}^{m_{j_{v}}}\right) \stackrel{f_{*}}{\longrightarrow}\left(T, T_{l}\right) \longrightarrow(T, N) \longrightarrow 0$. Aplicando o funtor $T \otimes_{\Gamma}-$, obtemos, desde que $\operatorname{Tor}_{1}^{\Gamma}(T, N)=0$, (pois $N \in \mathcal{T}(T)$ ), a seqüência $0 \longrightarrow \oplus_{v} T_{j_{v}}^{m_{j_{v}}} \stackrel{f}{\longrightarrow} T_{l} \longrightarrow N \longrightarrow 0$, que é exata. Logo, $f$ é um monomorfismo.

Reciprocamente, se $f$ é um monomorfismo, então $f_{*}$ é monomorfismo. Logo, a seqüência exata curta, $0 \longrightarrow \oplus_{v} T_{j_{v}}^{m_{j v}} \longrightarrow T_{l} \longrightarrow$ conucf $\longrightarrow 0$, com conucf $\in \mathcal{T}(T)$. Assim, é imediato que $(T$, conucf $) \cong S_{l} \in \mathcal{Y}(T)$. 
(ii) Pela proposição 4.1 e por (i), é imediato que $S_{l} \in \mathcal{X}(T)$ se, e somente se, $\mathrm{f}$ é um epimorfismo. Aplicando o funtor $\operatorname{Hom}_{\Lambda}(T,-)$ a seqüência exata curta $0 \longrightarrow n u c f \longrightarrow \oplus_{v} T_{j_{v}}^{m_{j_{v}}} \longrightarrow T_{l} \longrightarrow 0$, segue imediatamente que $S_{l} \cong \operatorname{Ext}_{\Lambda}^{1}(T, n u c f)$, e que $n u c f \in \mathcal{F}(T)$, caso $\operatorname{pd} S_{l}=1$, e $n u c f \notin \mathcal{F}(T)$, caso $\operatorname{pd} S_{l}=2$.

Mantendo as notações e aplicando o corolário acima, podemos concluir que o núcleo de $f$ é dado por:

$$
K=\left\{\begin{array}{l}
N_{1} \text { ou } \\
N_{2} \text { ou } \\
\bar{T} \oplus N_{i} \text { para } i=1,2
\end{array}\right.
$$

onde $N_{1} \in$ ind $\mathcal{F}, N_{2} \in \Lambda$-mod é um módulo que não pertence à teoria de torção gerada por $T$ e $\bar{T} \in$ add $T$.

Com efeito. Se $S_{l} \in \mathcal{Y}(T)$, temos que $K=0$. Caso $S_{l} \in \mathcal{X}(T)$, então ou $K \in \mathcal{F}(T)$ ou $K \notin \mathcal{F}(T)$.

Como $\operatorname{Ext}_{\Lambda}^{1}(T, K) \cong k$, então $\operatorname{Ext}_{\Lambda}^{1}(T, K)$ é isomorfo a $\operatorname{Ext}_{\Lambda}^{1}\left(T, N_{1}\right)$ ou à $\operatorname{Ext}_{\Lambda}^{1}\left(T, N_{2}\right)$, para $N_{i}, i=1,2$, um dos módulos como definido acima. Observando que, no caso de termos $K \notin \mathcal{F}(T)$, ocorre, também, que $\operatorname{Hom}_{\Lambda}(T, K) \neq$ 0 , e como este último módulo é isomorfo à um $\Gamma$-módulo projetivo, segue nossa afirmação.

Neste momento, estaremos interessados em identificar os módulos $K=$ nuc $f$, tal que $K \notin \mathcal{F}$ pois caso contrário, temos $\Gamma$ hereditária, que já sabemos ser uma álgebra de Koszul.

Daqui para frente estaremos assumindo que pd $S_{l}=2$.

Consideremos a $\Gamma$-resolução projetiva minimal de $S_{l}$ dada por:

$$
0 \longrightarrow \bigoplus_{u=1}^{t} P_{l_{u}}^{d_{l_{u}}} \stackrel{\rho_{*}}{\longrightarrow} \bigoplus_{v=1}^{s} P_{j_{v}}^{m_{j_{v}}} \stackrel{f_{*}}{\longrightarrow} P_{l} \longrightarrow S_{l} \longrightarrow 0
$$

onde $P_{x}=\operatorname{Hom}_{\Lambda}\left(T, T_{x}\right)$ para cada índice $x$, e $f_{*}=\left(f_{i}^{*}, \ldots, f_{s}^{*}\right)$ como definimos no início desta seção, e

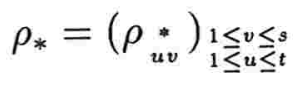

é induzida pelo $\Lambda$-morfismo $\left(\rho_{u v}\right)_{u, v} \operatorname{com} \rho_{u v}: T_{l_{u}}^{d_{l_{u}}} \longrightarrow T_{j_{v}}^{m_{j_{v}}}$ tal que $\rho_{u v}$ é a composição com $\rho_{u v}$, e $d_{l_{u}}$ é a multiplicidade de $P_{l_{u}}$ em $\Omega^{2}\left(S_{l}\right)$. 
Como $S_{l}$ é um módulo simples podemos assumir que cada função componente da apresentação $f_{*}$ de $S_{l}$ é induzida pela multiplicação por flechas $\alpha_{v}^{m_{v}}$ em $Q(\Gamma)$, ligando o vértice $l$ ao vértice $j_{v}$, onde $m_{v}=1, \cdots, m_{j_{v}}$, (cf Teo. 2.5 em [GM,1]). Observamos, também, que como $f_{v}: T_{j_{v}}^{m_{j_{v}}} \longrightarrow T_{l}$ é não nula, então temos que $T_{j_{v}}$ e $T_{l}$ são somandos diretos indecomponíveis de $T$, não isomorfos, pois a resolução acima é minimal e $\Gamma$ não contém circuitos orientados.

Como temos $f_{*} \circ \rho_{*}=0$, segue que $f \circ \rho=0$. Assim, se tormarmos a representação matricial destas aplicações, teremos a seguinte expressão:

$$
[f \circ \rho]=\left[f_{1} f_{2} \ldots f_{s}\right] \cdot\left[\begin{array}{ccc}
\rho_{11} & \cdots & \rho_{t 1} \\
\vdots & & \vdots \\
\rho_{1 s} & \cdots & \rho_{t s}
\end{array}\right]=\left[\sum_{v=1}^{s} f_{v} \rho_{1 v}, \ldots, \sum_{v=1}^{s} f_{v} \rho_{t v}\right]=[0]
$$

Ou seja, para cada $u=1, \ldots, t$ fixado, temos que $\sum_{v=1}^{s} f_{v} \rho_{u v}=0$, .

Observamos que como $\rho_{*}$ é um monomorfismo, temos que cada coluna da matriz $\left(\rho_{u v}^{*}\right)_{v}$ é um morfismo de $P_{l_{u}}^{d_{l_{u}}}$ em $\bigoplus_{v} P_{j_{v}}^{m_{j_{v}}}$, que é, também, um monomorfismo, para cada $u$ fixado. Mais claramente, o $\Gamma$-morfismo dado por $\left[\rho_{u 1}^{*}, \ldots, \rho_{u s}^{*}\right]: P_{l_{u}}^{d_{l_{u}}} \longrightarrow \bigoplus_{v=1}^{s} P_{j_{v}}^{m_{j_{v}}}$ é um monomorfismo.

Pelos resultados obtidos na proposição 4.1 e seu corolário, podemos concluir que:

(a) $S_{l} \cong \operatorname{Ext}_{\Lambda}^{1}(T$, nuc $f)$, com nuc $f \notin \mathcal{F}(T)$.

(b) $\operatorname{Hom}_{\Lambda}(T$, nuc $f) \cong \bigoplus_{u} P_{l_{u}}^{m_{l_{u}}}$

(c) $\rho_{*}$ está induzido pelo monomorfismo $\rho$ tal que:

$$
0 \longrightarrow K=\operatorname{nuc} f \stackrel{\rho}{\longrightarrow} \bigoplus T_{j_{v}}^{m_{j_{v}}} \stackrel{f}{\longrightarrow} T_{l} \longrightarrow 0
$$

Finalmente, considerando o monomorfismo de $\Lambda$-módulos $\rho$, obteremos a seqüência exata curta $0 \longrightarrow \bigoplus_{u} T_{l_{u}}^{d_{l_{u}}} \stackrel{\rho^{\prime}}{\longrightarrow} \bigoplus_{v} T_{j_{v}}^{m_{j_{v}}} \longrightarrow$ conuc $\rho^{\prime} \longrightarrow 0$, onde $\rho^{\prime}=\rho \circ i \operatorname{com} i=\operatorname{Tr}_{T}(K) \hookrightarrow K$ dado pela inclusão canônica.

Aplicando $\operatorname{Hom}_{\Lambda}(T,-)$ à esta seqüência vamos obter a seqüência exata curta de $\Gamma$-módulos $0 \longrightarrow \bigoplus_{u} P_{l_{u}}^{d_{l_{u}}} \stackrel{\rho_{*}}{\longrightarrow} \bigoplus_{v} P_{j_{v}}^{m_{j_{v}}} \longrightarrow\left(T\right.$, conuc $\left.\rho^{\prime}\right) \longrightarrow 0$. 
Comparando esta sequência com a $\Gamma$-resolução projetiva minimal de $S_{l}$, que fixamos inicialmente, podemos concluir que $\left(T\right.$, conuc $\left.\rho^{\prime}\right) \cong r_{\Gamma} P_{l}$.

\subsection{A componente de grau zero de $\Gamma$}

Estaremos interessados em estudar, nesta seção, os morfismos de grau zero entre os somandos diretos indecomponíveis de um módulo inclinante $T$, graduado, gerado em grau zero sobre uma $k$-álgebra de dimensão finita, hereditária, $\Lambda$.

Supondo que $\Gamma=\operatorname{End}_{\Lambda}(T)^{o p}$ é um quociente de uma álgebra de caminhos por um ideal homogêneo, então poderemos considerar que $\Gamma^{(0)}$, a componente de grau zero de $\Gamma$, é um produto finito de cópias de $k$, gerada pelo conjunto dos idempotentes ortogonais primitivos de $\Gamma$. No caso em que não tivermos informações sobre o ideal das relações, passamos a considerar sobre $\Gamma$ a graduação definida pelas morfismos entre os somandos diretos de $T$, conforme foi definido na seção 4.3. Nesta situação, obtivemos algumas informações sobre $\Gamma$, analisando a componente de grau zero $\Gamma^{(0)}$.

Para apresentar estes resultados precisaremos introduzir notações e alguns fatos sobre as morfismos de grau zero, entre inclinantes parciais indecomponíveis.

Comecemos tomando $v$ no conjunto de indices $\{1, \ldots, n\}$ e consideremos $\operatorname{Hom}_{\Lambda}\left(T, T_{v}\right)$ como um $\mathbb{Z}$-módulo graduado, com a graduação dada pelo grau dos morfismos, como fizemos na seção 4.3, a saber,

$$
\operatorname{Hom}_{\Lambda}\left(T, T_{v}\right)=\bigoplus_{m \geq 0} F\left[\operatorname{Homgr}(\Lambda)\left(T, \sigma(-m) T_{v}\right)\right]
$$

onde $T_{v}$ é um somando direto indecomponível de $T$.

Pelo lema 4.1, pag 419, em [HR], sabemos que sobre uma álgebra hereditária, os morfismos não-nulos entre módulos indecomponíveis inclinantes parciais, ou são epimorfismos ou são monomorfismos. Este resultado vai direcionar nossas argumentações; assim é crucial considerar álgebras inclinadas, ao invés da situação mais geral apresentada no parágrafo 4.3.

Seja $f: T_{u} \longrightarrow T_{v}$ um morfismo homogêneo não-nulo, com $u \neq v$, no conjunto de índices fixado acima. Observamos que, se $T_{v}$ é um $\Lambda$-módulo simples então $f$ é necessariamente um epimorfismo. Por outro lado, se $f$ 
é um epimorfismo, teremos que $f\left(T_{u}^{(0)}\right)=T_{v}^{(0)}$, já que $T_{u}$ e $T_{v}$ são gerados em grau zero. Segue que $f$ tem grau zero. Assim, se $f \in \operatorname{Hom}_{\Lambda}\left(T_{u}, T_{v}\right)$ é um morfismo graduado não-nulo, homogêneo de grau $m>0$, então $f$ é um monomorfismo graduado.

Os próximos resultados nos dizem o que ocorre com $\Gamma=\operatorname{End}_{\Lambda}(T)^{o p}$, ao impormos certas condições sobre sua componente de grau zero. Vejamos como.

Proposição 4.3 Sejam $\Lambda$ uma k-álgebra de dimensão finita, hereditária, $e$ $T$ um $\Lambda$-módulo graduado, inclinante, gerado em grau zero, livre de multiplicidade, dado por $T=\bigoplus_{j=1}^{n} T_{j}$, onde cada $T_{j}$ é indecomponível. Consideremos $\Gamma=\operatorname{End}_{\Lambda}(T)$. Se Homgr $(\Lambda)\left(T_{i}, T_{j}\right)=0$, para todo $i \neq j$ então $\Gamma$ é hereditária.

Demonstração: Consideremos $S_{l}$ um $\Gamma$-módulo simples, não projetivo e sua gr $\Gamma$-apresentação projetiva minimal

$$
\bigoplus_{v=1}^{s} P_{j_{v}}^{m_{j_{v}}} \stackrel{f_{*}}{\longrightarrow} P_{l} \longrightarrow S_{l} \longrightarrow 0
$$

onde $f_{*}$ é induzida por $f=\left(f_{1}, \ldots, f_{s}\right)$ com $f_{v}: T_{j_{v}}^{m_{j_{v}}} \longrightarrow T_{l}$ tal que $f_{v}$ é a composição com $f_{v}$.

Como $\Gamma$ não possue ciclos orientados, sabemos que $l \neq j_{v}$, para todo $v=1, \ldots, s$. Por hipótese, não existem morfismos graduados entre somandos diretos de $T$, não isomorfos. Segue que cada função componente de $f_{v}: T_{j_{v}}^{m_{j_{v}}} \longrightarrow T_{l}$ é um morfismo homogêneo de grau positivo, para cada $v=1, \ldots, s$. E, portanto, $f_{v}$, também o é. Assumamos que cada função componente $f_{v}^{m_{v}}$ de $f_{v}$ é um monomorfismo de grau positivo $r_{m_{v}}$, com $m_{v}=1, \ldots, m_{j_{v}}$. Como $f_{*}$ é a apresentação de um módulo simples em $\Gamma$ segue que $r_{m_{v}}=r_{0}$ fixado, para cada $m_{v}=1, \ldots, m_{j_{v}}$ e $v=1, \cdots, s$.

Assim, temos que $\operatorname{Im} f \subset \underset{r \geq r_{0}}{\bigoplus} T_{l}^{(r)}$. Logo, $f$ não é um epimorfismo. Segue pela proposição 4.1 que $f$ é um monomorfismo. Logo $f_{*}$ é um monomorfismo e portanto pd $S_{l}=1$. Segue que gldim $\Gamma=1$. 
Observamos que não vale a recíproca desta proposição. Para ver isso, considere o exemplo 2 da seção 4.3 , onde temos que $\Gamma$ é hereditária e gerada em grau zero, na graduação induzida pelo grau dos morfismos.

A proposição a seguir direciona o comportamento da componente de grau zero de $\Gamma$, quando esta é uma álgebra monomial.

Proposição 4.4 Sejam $\Lambda$ uma k-álgebra de dimensão finita, hereditária e $T=\oplus_{j=1}^{n} T_{j}$ um $\Lambda$-módulo inclinante, graduado, gerado em grau zero e livre de multiplicidade. Seja $\Gamma=\operatorname{End}_{\Lambda}(T)$, e consideremos a gr $\Gamma$-apresentação projetiva minimal de $\Gamma / r$

$$
\bigoplus_{v} P_{j_{v}}^{m_{j_{v}}} \stackrel{f_{*}}{\longrightarrow} \Gamma \longrightarrow \Gamma / r \longrightarrow 0
$$

Se $\Gamma$ é monomial então para cada $v$, existe um somando direto indecomponível $T^{\prime}$ de $T$, com $T^{\prime} \neq T_{j_{v}}$, tal que Homgr ${ }_{\Lambda}\left(T_{j_{v}}, T^{\prime}\right) \neq 0$.

Demonstração: Seja a gr $\Gamma$-resolução projetiva minimal de $\Gamma / r$

$$
0 \longrightarrow P_{(2)} \stackrel{\rho_{*}}{\longrightarrow} P_{(1)}=\bigoplus_{v} P_{j_{v}}^{m_{j_{v}}} \stackrel{f_{*}}{\longrightarrow} \Gamma \longrightarrow \Gamma / r \longrightarrow 0
$$

Sem perda de generalidade, assumiremos que $P_{(2)} \cong \bigoplus_{u} \operatorname{Hom}_{\Lambda}\left(T, T_{l_{u}}\right)^{m_{l_{u}}}$, onde os módulos $T_{l_{u}}$ são somandos diretos indecomponíveis de $T$, não isomorfos dois a dois. Um cálculo rápido com as funções componentes de $f_{*} \mathrm{e}$ $\rho_{*}=\left(\rho_{u v}^{*}\right)_{u, v}$, nos mostra que cada entrada da matriz $\left[\sum_{v=1}^{s} f_{v} \rho_{u v}\right]_{u}$ é nula, (cf. a seção 4.4).

Como $\Gamma$ é monomial e $P_{(2)}=I I_{I^{2}}$, onde $\Gamma=k Q / I$, segue que cada vértice $u$, associado ao projetivo $P_{l_{u}}$, é final de uma relação monomial de $\Gamma$, (cf em [B]). Assim, podemos assumir que $f_{v} \rho_{u v}=0$, para cada par $(u, v)$ fixado. Consideremos, agora, as funções componentes de $f_{v}$ e $\rho_{u v}$, dadas por $f_{v}=\left(f_{v}^{1}, \cdots, f_{v}^{m_{j_{v}}}\right): T_{j_{v}}^{m_{j_{v}}} \longrightarrow T_{l}$, onde $f_{v}^{m_{v}}$ é um morfismo de $T_{j_{v}}^{m_{j_{v}}}$ em $T_{l}$, e $\rho_{u v}=\left(\rho_{u v}^{1}, \cdots, \rho_{u v}^{d_{l u}}\right): T_{l_{u}}^{d_{l_{u}}} \longrightarrow T_{j_{v}}^{m_{j_{v}}}$, onde $\rho_{u v}^{d_{u}}$ é um morfismo de $T_{l_{u}}$ em $T_{j_{v}}^{m_{j v}}$, para $d_{u}=1, \cdots, d_{l_{u}}$. Assim, como temos que $f_{v} \rho_{u v}=0$, segue que $f_{v} \cdot\left(\rho_{u v}^{1}, \cdots, \rho_{u v}^{d_{l u}}\right)=0$, para cada par $(u, v)$ fixado. Portanto, temos que $f_{v} . \rho_{u v}^{d_{u}}=0$, para cada $d_{u}=1, \cdots, d_{l_{u}}$. Finalmente, temos que $f_{v}^{m_{v}} \rho_{u v}^{d_{u}}=0$, para cada $m_{v}=1, \cdots, m_{j_{v}}, d_{u}=1, \cdots, d_{l_{u}}$ onde $(u, v)$ é um par fixado. 
Então, pelo Lema 4.1 em [HR], segue imediatamente que $f_{v}^{m_{v}}$ é um epimorfismo, para cada $m_{v}$. Como $T$ é módulo graduado, gerado em grau zero, segue que $f_{v}^{m_{v}}$ é morfismo homogêneo de grau zero. Portanto, existem morfismos graduados entre somandos diretos indecomponíveis de $T$ não isomorfos.

\section{Observação:}

Notemos que no caso das álgebras $\mathrm{BB}$-inclinadas $\Gamma=\Gamma_{i}$, a apresentação do semi-simples $\Gamma / r$ é induzida por um morfismo tal que cada função componente é um monomorfismo de grau zero. Com efeito, a $\Lambda$-resolução projetiva de $\tau^{-} S_{i}$, dada por

$$
0 \longrightarrow P_{i} \longrightarrow \bigoplus_{s} P_{l_{s}}^{m_{s}} \stackrel{\left(f_{s}\right)}{\longrightarrow} \tau^{-} S_{i} \longrightarrow 0
$$

induz a resolução projetiva de $\hat{S}$ e cada morfismo $f_{s}$ é homogêneo de grau zero e é um monomorfismo. (Para mais detalhes, cf. seção 3.2).

\subsection{A cobertura projetiva de $\operatorname{rad} \Gamma$}

Nosso objetivo neste parágrafo, é definir as aplicações entre os somandos diretos indecomponíveis do inclinante $T$, tais que os vértices da aljava $Q(\Gamma)$, associados aos $\Gamma$-módulos projetivos indecomponíveis determinados por estes somandos, estejam ligados por flechas.

Definição 4.5 Seja $f: T_{v} \longrightarrow T_{l}$ um morfismo não-nulo, com $T_{j} \not T_{v}$. Dizemos que $f$ não se fatora própriamente através de addT, se $f=g h$ para algum $g: T^{\prime} \longrightarrow T_{l}$ e $h: T_{v} \longrightarrow T^{\prime}$, com $T^{\prime} \in$ addT, então ou $h$ é um monomorfismo que cinde ou $g$ é um epimorfismo que cinde.

Seja dado $T=\oplus_{j=1}^{n} T_{j}$, módulo inclinante sobre $\Lambda$ uma $k$-álgebra de dimensão finita, hereditária. Fixemos $P=P_{l}=\operatorname{Hom}_{\Lambda}\left(T, T_{l}\right)$, tal que $r P_{l} \neq$ 0 , e $T_{l}$ é um somando direto indecomponível de $T$. Consideremos $P_{(0)}\left(r P_{l}\right)=$ $\bigoplus_{m} P_{m}$, uma decomposição em projetivos indecomponíveis para a cobertura 
projetiva de $r_{\Gamma} P_{l}$. Observamos que devemos ter $\operatorname{Hom}_{\Lambda}\left(T_{m}, T_{l}\right) \neq 0$, para $m \neq l$, como definidos acima.

Lema 4.6 Sejam $P_{l}=\left(T, T_{l}\right)$ um $\Gamma$-módulo projetivo indecomponível e $j \neq l$ tal que $\left(T_{j}, T_{l}\right) \neq 0$. Então, $P_{j}$ é somando direto de $P_{(0)}\left(r P_{l}\right)$ se e somente se, existe um morfismo não nulo $f: T_{j} \longrightarrow T_{l}$, que não se fatora própriamente através de addT.

Demonstração: Suponhamos que $P_{j}=\left(T, T_{j}\right)$ seja um somando direto de $P_{(0)}\left(r P_{l}\right)$. Então, existe um morfismo não nulo $f_{*}: P_{j} \longrightarrow P_{l}$, tal que $\operatorname{Im} f_{*} \subset r P_{l}$ e $\operatorname{Imf} \not \subset r^{2} P_{l}$.

Seja $f: T_{j} \longrightarrow T_{l}$ morfismo, não nulo, que induz $f_{*}$. Afirmamos que $f$ não se fatora propriamente através de add $T$. Caso contrário, existem $g: T^{\prime} \longrightarrow T_{l}$ e $h: T_{j} \longrightarrow T^{\prime}$, não nulos, com $T^{\prime} \in \operatorname{add} T$, tal que $f=g h$, onde $g$ não é epimorfismo que cinde e $h$ não é monomorfismo que cinde. Portanto, temos, pela BB-equivalência que $f_{*}=g_{*} h_{*}$, com $g_{*}$ não epimorfismo que cinde e $h_{*}$ não monomorfismo que cinde. Logo, $I m g_{*} \subset r P_{l}$ e $I m h_{*} \subset r\left(T, T^{\prime}\right)$, resultando que $I m f_{*} \subset r^{2} P_{l}$, o que contraria a hipótese.

Reciprocamente, suponhamos que exista um morfismo, não nulo, $f$ : $T_{j} \longrightarrow T_{l}$ que não se fatora propriamente através de $\operatorname{add} T$. Mostremos que $P_{j}$ é somando direto de $P_{(0)}\left(r P_{l}\right)$. Suponhamos que este não seja o caso. Então, todo morfismo não nulo, de $P_{j}$ para $P_{l}$ tem imagem em $r^{2} P_{l}$. Em particular, temos que $\operatorname{Im} f_{*} \subset r^{2} P_{l}$. Então $f_{*}$ é uma combinação linear de funções $f_{\gamma_{s}}$ determinadas por caminhos $\gamma_{s} \in Q(\Gamma)$, que não estãono ideal de relações, de comprimento maior ou igual a dois, e que ligam o vértice $l$ (associado ao projetivo $P_{l}$ ) ao vértice $j$ (associado ao projetivo $P_{j}$ ). Usando a BB-equivalência, resulta que $f$ é uma combinação linear das funções $f_{\gamma_{s}}$, que induzem as $f_{\gamma_{s}}$. Desde que $f$ não se fatora propriamente através de add $T$, temos que existe $i_{0}$ tal que $f_{\gamma}=f_{\gamma_{i_{0}}}$ não se fatora propriamente através de $\operatorname{add} T$. Como $\gamma$ é um caminho de comprimento maior ou igual a dois, podemos escrever $\gamma=\gamma_{1} \cdot \gamma_{2}$, onde $\gamma_{1}$ e $\gamma_{2}$ são caminhos de comprimento maior ou igual a um. Sabemos que os caminhos $\gamma_{1}$ e $\gamma_{2}$ induzem aplicações entre os projetivos associados aos vértices iniciais e finais destes. Assim, temos um 
diagrama comutativo dado por:

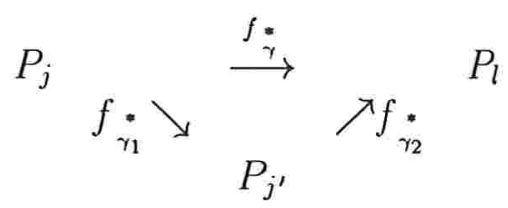

onde $f_{\gamma^{*}}, f_{\gamma_{1}}$ e $f_{\gamma_{2}}$ são os morfismos determinados pelos caminhos $\gamma, \gamma_{1}$ e $\gamma_{2}$, respectivamente, e $j^{\prime}$ é o vértice tal que $j^{\prime}=s\left(\gamma_{2}\right)=e\left(\gamma_{1}\right)$.

Pela equivalência de Brenner-Butler, obteremos um diagrama equivalente em $\Lambda$-mod dado por:

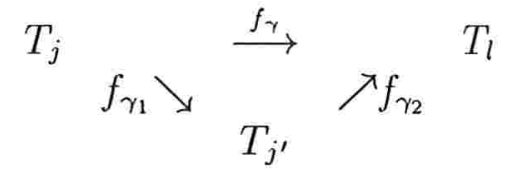

tal que $f_{\gamma}=f_{\gamma_{2}} \circ f_{\gamma_{1}} \neq 0$, onde ou $f_{\gamma_{1}}$ é um monomorfismo que cinde ou $f_{\gamma_{2}}$ é um epimorfismo que cinde. Logo, $I m f_{\dot{\gamma}} \subset r P_{l}$ e $I m f_{*} \not \subset r^{2} P_{l}$, pois ou $f_{\gamma_{1}}$ é um monomorfismo que cinde ou $f_{\gamma_{2}}$ é um epimorfismo que cinde, contrariando a hipótese de não ser $P_{j}$ um somando direto de $P_{(0)}\left(r P_{l}\right)$.

Podemos traduzir as idéias e parte de nossos resultado obtido no lema acima, para o caso dos caminhos diretos não nulos, o que mostraremos em seguida.

Definição 4.7 Dados $A$ e $B$, dois $\Lambda$-módulos indecomponíveis, chamamos de caminho direto de $A$ para $B$, a uma seqüência qualquer de morfismos não nulos, não isomorfismos

$$
A_{0}=A \stackrel{f_{1}}{\longrightarrow} A_{1} \longrightarrow \cdots \longrightarrow A_{n} \stackrel{f_{n+1}}{\longrightarrow} A_{n+1}=B
$$

onde $A_{j}$ são $\Lambda$-módulos indecomponiveis, para cada $j$. Se a composição de aplicações $f=f_{n+1} \circ \cdots \circ f_{1}$ é não-nula, então dizemos que o caminho direto é não-nulo.

Corolário 4.8 Sejam $P_{l}=\left(T, T_{l}\right)$ e $j \neq l$, fixados, tais que $\left(T_{j}, T_{l}\right) \neq 0$. Suponhamos que para todo caminho direto não-nulo de $T_{j}$ para $T_{l}$, somente os extremantes $T_{j}$ e $T_{l}$ estão em addT. Então $P_{j}$ é um somando direto de $P_{(0)}\left(r P_{l}\right)$. 
Demonstração: Imediata.

É fato conhecido que sobre álgebras hereditárias, todo morfismo não-nulo, entre módulos preprojetivos é soma de composições de morfismos irredutíveis. No caso em que $T$ é um módulo preprojetivo, sobre a álgebra hereditária de dimensão finita, $\Lambda$, chamamos $\Gamma$ de álgebra disfarçada. Neste caso, podemos nos orientar pela aljava de $A R$ de $\Gamma$ para encontrar os morfismos não-nulos entre os módulos indecomponíveis, somandos diretos de $T$, pois, os morfismos entre módulos preprojetivos indecomponíveis são soma de composições de morfismos irredutíveis. O exemplo a seguir esclarece o que queremos dizer.

Exemplo: Seja $\Lambda$ a álgebra dada por:

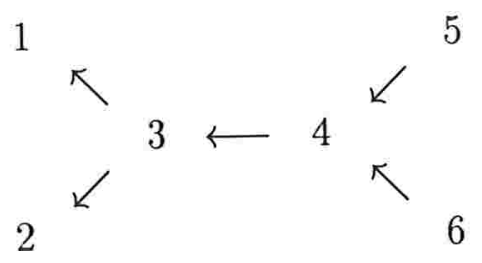

e considere $T=\tau^{-2} S_{1} \oplus \tau^{-} S_{2} \oplus P_{6} \oplus P_{5} \oplus P_{4} \oplus P_{1}$ um $\Lambda$-módulo inclinante.

Um esboço local da aljava de AR de $\Lambda$ é dado por:

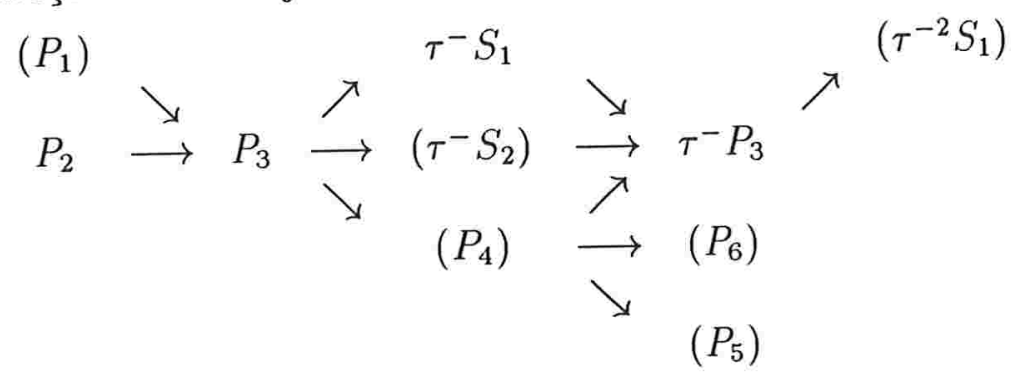

onde os módulos entre parênteses são os somandos diretos indecomponíveis de $T$, e as flechas indicam os morfismos irredutíveis. Os caminhos não-nulos entre os somandos diretos indecomponíveis de $T$, na aljava de AR de $\Lambda$, desenham a seguinte aljava:

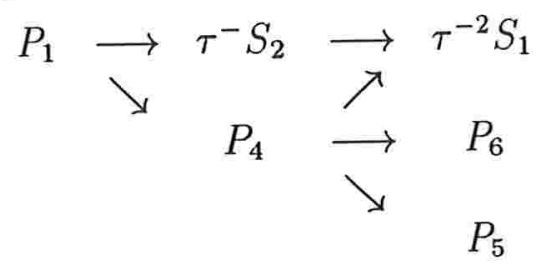


onde as flechas indicam morfismos não nulos, que não são isomorfismos.

Um cálculo simples mostra que $Q(\Gamma)$ é dada por:

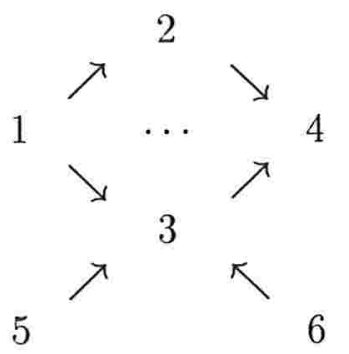

onde $\hat{P}_{1}=\left(T, \tau^{-2} S_{1}\right), \quad \hat{P}_{2}=\left(T, \tau^{-} S_{2}\right), \quad \hat{P}_{3}=\left(T, P_{4}\right), \quad \hat{P}_{4}=\left(T, P_{1}\right)$, $\hat{P}_{5}=\left(T, P_{5}\right)$ e $\hat{P}_{6}=\left(T, P_{6}\right)$ e as relações sobre $Q(\Gamma)$ são de comutatividade,

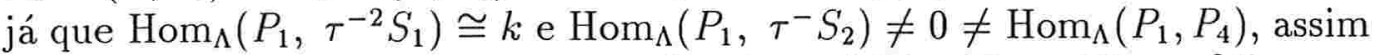
como são não-nulos os grupos $\operatorname{Hom}_{\Lambda}\left(\tau^{-} S_{2}, \tau^{-2} S_{1}\right)$ e $\operatorname{Hom}_{\Lambda}\left(P_{4}, \tau^{-2} S_{1}\right)$.

\subsection{Aplicações poço de torção}

Nesta seção, vamos introduzir um novo conceito para aplicações entre módulos de torção, que nos levará, no parágrafo seguinte, a apresentar nosso principal resultado neste capítulo, que é caracterizar as álgebras inclinadas graduadas que são álgebras de Koszul. Enfatizamos que, neste parágrafo, estaremos considerando $\Lambda$ uma $k$-álgebra de dimensão finita, não necessáriamente hereditária.

Definição 4.9 Sejam $M$ e $N, \Lambda$-módulos com $M$ e $N \in \mathcal{T}(T)$ e $M$ indecomponível. Dizemos que um morfismo $\alpha: N \longrightarrow M$, não nulo, é um morfismo poço de torção, ou simplesmente um poço-torção, quando a não é um epimorfismo que cinde e todo morfismo não-nulo, que não seja epimorfismo que cinde, $\beta: L \rightarrow M$, com $L \in \mathcal{T}(T)$, se fatora através de $\alpha$. Ou seja, um poço de torção é um morfismo poço na subcategoria plena $\mathcal{T}(T)$.

Vejamos alguns exemplos.

Exemplo 1: Todo morfismo que seja um morfismo minimal quase cindido à direita, e tal que ambos os módulos envolvidos sejam módulos em $\mathcal{T}(T)$ é um poço-torção. 
Exemplo 2: Seja $M \in \mathcal{T}(T)$ indecomponível e considere $f: E \longrightarrow M$ a aplicação quase cindida à direita de $M$. Tomemos $\operatorname{tr}_{T}(E) \in \mathcal{T}(T)$ o submódulo maximal de torção de $E$, e consideremos o seguinte diagrama:

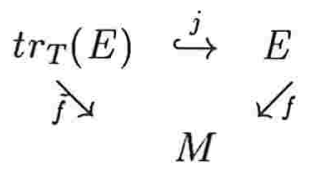

onde $j$ é a inclusão canônica e $\tilde{f}=f \circ j$. Então, $\tilde{f}$ é um morfismo poço de torção.

De fato, seja $\beta: L \longrightarrow M$ um morfismo não-nulo, que não seja um epimorfismo que cinde, $\operatorname{com} L \in \mathcal{T}(T)$. Então, $\beta$ se fatora através de $f$. Digamos que seja da seguinte maneira:

$$
\begin{array}{cc}
L \stackrel{\beta}{\longrightarrow} \quad M \\
h \searrow \quad \begin{array}{c}
\uparrow f \\
\end{array}
\end{array}
$$

$\operatorname{com} \beta=f \circ h$. Como $\operatorname{Im} h \in \mathcal{T}(T)$, pois esta classe de módulos é fechada por imagens, segue que $\operatorname{Im} h \subseteq \operatorname{tr}_{T}(E)$. Portanto, temos que $\beta=f \circ j \circ h$. Ou seja, $\beta$ se fatora através de $\tilde{f}$. Mas, como temos que $f$ não é epimorfismo que cinde segue que $\tilde{f}$ não será epimorfismo que cinde. Portanto, temos que $\tilde{f}$ é um poço-torção.

Definição 4.10 Dizemos que um poço-torção $\alpha: N \longrightarrow M$ é minimal se $\alpha$ é minimal à direita, ou seja, todo morfismo $g: N \longrightarrow N$, tal que $\alpha=\alpha \cdot g$, é um isomorfismo.

Observamos que esta definição tem o mesmo sentido que é dado em [ARS], para os morfismos quase cindidos minimais.

O próximo resultado mostrará que são os morfismos poço de torção em $\Lambda$, cujas componentes não se fatoram própriamente por somandos diretos de $T$, aqueles que induzem flechas na aljava de $\Gamma$. Mais precisamente, mostraremos que estas aplicações poço de torção, que tem como contradomínio os somandos diretos indecomponíveis de $T$, vão induzir um conjunto de morfismos, 
que pertencem ao $r \Gamma$ mas não pertencem ao $r^{2} \Gamma$. Decorre disto que, ao considerarmos o teorema de Gabriel, da maneira como explicitamos no capítulo de introdução, teremos obtido um conjunto de morfismos que contém aqueles que são induzidos pela multiplicação por flechas da aljava de $\Gamma$.

Proposição 4.11 Sejam $\Lambda$ uma k-álgebra de dimensão finita e $T$ um $\Lambda$ módulo inclinante. Seja $T_{l}$ um somando direto indecomponível de $T$, tal que tenhamos $P_{l}=\left(T, T_{l}\right)$ um $\Gamma$-módulo não simples. Então, existe um único, a menos de isomorfismo, morfismo poço de torção minimal, $\alpha: M \longrightarrow T_{l}$.

Mais ainda, $M \cong T \otimes r P_{l}$ e $\alpha=T \otimes g_{*}$, onde $g_{*}: r P_{l} \longrightarrow P_{l}$ é o morfismo poço minimal em $\Gamma$-mod.

Demonstração: Desde que $T_{l}$ é somando direto indecomponível de $T$, tal que $P_{l}$ é um $\Gamma$-módulo projetivo indecomponível, não simples, temos que $r P_{l} \neq 0$. Logo, existe $\alpha^{*}: r P_{l} \longrightarrow P_{l}$, morfismo poço, minimal em $\Gamma$-mod. Como $r P_{l} \in \mathcal{Y}(T)$, existe $M \in \mathcal{T}(T)$ tal que $r P_{l} \cong \operatorname{Hom}_{\Lambda}(T, M)$. Segue que $\alpha_{*}$ é induzido por um morfismo não-nulo $\alpha: M \longrightarrow T_{l}$. Afirmamos que $\alpha$ é um poço de torção. Claramente, $\alpha$ não é um epimorfismo que cinde, pois caso isto ocorresse teríamos que $\alpha^{*}$ seria um morfismo que cinde, um absurdo. Sejam $N \in \mathcal{T}(T)$ e o morfismo não nulo $\beta: N \longrightarrow T_{l}$, que não é um epimorfismo que cinde. Então, $\beta_{*}:(T, N) \longrightarrow\left(T, T_{l}\right)$ não é epimorfismo que cinde. Segue que $\beta_{*}$ se fatora através de $\alpha_{*}$, ou seja existe $h_{*}:(T, N) \longrightarrow r P_{l}$ um morfismo não nulo tal que $\beta_{*}=\alpha_{*} h_{*}$. Logo, $\beta=\alpha h$, pela BB-equivalência. Segue que $\alpha$ é um poço-torção. O fato de $\alpha_{*}$ ser minimal implica, pela BB-equivalência, que $\alpha$ é também minimal.

Usando argumentos análogos aos apresentados acima, podemos concluir que um outro poço-torção minimal, qualquer, $\beta: N \longrightarrow T_{l}$, induz um morfismo poço $\beta_{*}$, que é morfismo poço minimal em $\Gamma$-mod. Logo, pela unicidade de $\alpha_{*}$ ( a menos de isomorfismo), temos que $\operatorname{Hom}_{\Lambda}(T, M) \cong r P_{l} \cong$ $\operatorname{Hom}_{\Lambda}(T, N)$, e portanto, $M \cong N$ pois ambos pertencem a $\mathcal{T}(T)$. Utilizando o funtor $T \otimes-$, obtemos que $M \cong T \otimes r P_{l} \cong$ $T \otimes_{\Gamma} \operatorname{Hom}_{\Lambda}(T, M)$ e que $\alpha=T \otimes \alpha_{*}$.

Corolário 4.12 Nas mesmas condições da Proposição 4.11, temos que o poço de torção minimal $\alpha: M \longrightarrow T_{l}$ ou é um monomorfismo ou é um epimorfismo. 
Demonstração: Suponha que $\alpha$ não é um epimorfismo. Então, $\operatorname{Im} \alpha \nsubseteq T_{l}$.

Como $\alpha$ é poço-torção e $\operatorname{Im} \alpha \in \mathcal{T}(T)$, segue que a inclusão $j: \operatorname{Im} \alpha \longrightarrow T_{l}$ se fatora através de $\alpha$. Então, $\operatorname{Im} \alpha$ é um somando direto de $M$. Ou seja, $M \cong$ nuc $\alpha \oplus \operatorname{Im} \alpha$. Se $\alpha$ não é um monomorfismo, então nuc $\alpha \neq 0$; como $M \in \mathcal{T}(T)$, segue que nuc $\alpha \in \mathcal{T}(T)$ e portanto, $r P_{l} \cong(T$, nuc $\alpha) \oplus(T, \operatorname{Im} \alpha)$, $\operatorname{com}(T$, nuc $\alpha) \neq 0$. Mas, $\alpha$ é minimal, e portanto, $\alpha_{*}$ é minimal. Como temos que $\alpha_{*}(T, n u c \alpha)=0$, obtemos uma contradição.

Corolário 4.13 Nas mesmas condições da Proposição 4.11, seja $\alpha: M \longrightarrow$ $T_{l}$ poço de torção mínimal. Então, $M \in$ add $(T)$ se e somente se $r P_{l}$ é um $\Gamma$-módulo projetivo.

Demonstração: É uma conseqüência imediata da demonstração da Proposição 4.11, e da unicidade do poço de torção minimal.

O próximo corolário descreve os $\Gamma$-módulos simples $S_{l}$, o topo dos projetivos indecomponíveis $P_{l}$, através dos morfismos poço de torção.

Corolário 4.14 Nas condições da Proposição 4.11, seja $\alpha: M \longrightarrow T_{l}$ o poşo-torção minimal para $T_{l}$. Seja $S_{l}$ o $\Gamma$-módulo simples do topo de $P_{l}$. Então, ou $S_{l} \cong \operatorname{Hom}_{\Lambda}\left(T, T_{l} / M\right)$ ou $S_{l} \cong \operatorname{Ext}_{\Lambda}^{1}(T$, nuс $\alpha)$, com nuс $\alpha \in \mathcal{F}(T)$.

Demonstração: Pelo Corolário 4.12, ou $\alpha$ é monomorfismo ou é epimorfismo. Na primeira situação, ou seja, se $\alpha$ é um monomorfismo, temos que a sequiência exata de $\Lambda$-módulos, $0 \longrightarrow M \stackrel{\alpha}{\longrightarrow} T_{l} \longrightarrow$ conuc $\alpha \longrightarrow 0$, define a seguinte seqüência exata de $\Gamma$-módulos, ao aplicarmos o funtor $\operatorname{Hom}_{\Lambda}(T,-)$,

$$
0 \longrightarrow(T, M) \stackrel{\alpha_{*}}{\longrightarrow} P_{l} \longrightarrow(T, \text { conuc } \alpha) \longrightarrow 0
$$

Pela demonstração da Proposição 4.11, $r P_{l} \cong(T, M)$, portanto $S_{l} \cong$ $\operatorname{Hom}_{\Lambda}\left(T, T_{l} / M_{M}\right)$.

Se $\alpha$ é um epimorfismo, temos a seguinte seqüência exata de $\Lambda$-módulos, $0 \longrightarrow$ nuc $\alpha \longrightarrow M \stackrel{\alpha}{\longrightarrow} T_{l} \longrightarrow 0$. Ao aplicarmos o funtor $\operatorname{Hom}_{\Lambda}(T,-) \grave{a ̀ ~}$ esta seqüência, obtemos a seqüência exata longa de $\Gamma$-módulos,

$$
0 \longrightarrow(T, \text { nuc } \alpha) \longrightarrow(T, M) \stackrel{\alpha_{*}}{\longrightarrow} P_{l} \longrightarrow \operatorname{Ext}_{\Lambda}^{1}(T, \text { nuc } \alpha) \longrightarrow 0
$$


Mas, $\alpha_{*}$ é um monomorfismo, logo $(T$, nuc $\alpha)=0$ e conseqüentemente nuc $\alpha \in \mathcal{F}(T)$ e $S_{l} \cong \operatorname{Ext}_{\Lambda}^{1}(T$, nuc $\alpha)$.

Como conseqüência do Corolário 4.14 , temos que ou pd $S_{l} \leq \operatorname{pd}_{\Lambda} T_{l} / M$ ou pd $S_{l} \leq 1+\operatorname{pd}_{\Lambda}$ nuc $\alpha$. Assim, se $\Lambda$ é hereditária, então $\Gamma=\operatorname{End}_{\Lambda}(T)^{o p}$ é hereditária se, e somente se, $\operatorname{pd}_{\Lambda}$ nuc $\alpha=0$, para cada $\alpha$ poço de torção minimal, definido por somandos diretos indecomponíveis de $T$, que não correspondam a $\Gamma$-módulos projetivos simples.

Corolário 4.15 Consideremos $\alpha: M \longrightarrow T_{l}$ o morfismo poço de torção minimal, obtido na Proposição 4.11. Suponhamos que o $\Gamma$-módulo simples $S_{l} \cong \frac{P_{l}}{r P_{l}}$, tenha dimensão projetiva dois e que o ideal da apresentação de $\Gamma$ seja graduado. Tomemos a gr( $\Gamma)$-apresentação projetiva minimal de $S_{l}$ dada por:

$$
\bigoplus_{v=1}^{s} P_{j_{v}}^{m_{j_{v}}} \stackrel{f_{*}}{\longrightarrow} P_{l} \longrightarrow S_{l} \longrightarrow 0
$$

Se cada aplicação $\rho_{v}^{\prime}: \operatorname{tr}_{T}($ nuc $f) \longrightarrow T_{j_{v}}$, para $v=1, \ldots, s$, é um poçotorção então $S_{l}$ é um módulo de Koszul.

Demonstração: Pela Proposição 4.11, existe $\alpha: M_{v} \longrightarrow T_{j_{v}}$ o morfismo poço de torção minimal, para cada $v=1, \cdots, s, \operatorname{com} r P_{j_{v}} \cong \operatorname{Hom}_{\Lambda}\left(T, M_{v}\right)$. Temos que $\rho_{v}^{\prime}$ se fatora através de $\alpha$, pois esta é um poço-torção e, por hipótese, temos que $\alpha$ se fatora através de $\rho_{v}^{\prime}$. Ou seja, existem aplicações não-nulas:

$$
t r_{T}(\text { nuc } f) \stackrel{g}{\longrightarrow} M_{v} \stackrel{h}{\longrightarrow} \operatorname{tr}_{T}(\text { nuc } f)
$$

tal que $\alpha_{v}=\alpha_{v} g h$. Logo, $g h$ é isomorfismo, já que $\alpha_{v}$ é minimal. Segue que $h$ é monomorfismo que cinde. Ou seja, $M_{v}$ é um somando direto de $\operatorname{tr}_{T}($ nuc $f)$ para cada $v=1, \ldots, s$. Mas, nuc $f_{*} \cong \operatorname{Hom}_{\Lambda}\left(T, \operatorname{tr}_{T}(\right.$ nuc $\left.f)\right)$ é um $\Gamma$-módulo projetivo. Portanto, $\bigoplus_{v} r P_{j_{v}}^{m_{j_{v}}}$ é um projetivo, somando direto de nuc $f_{*}$. Como nuc $f_{*} \subset \underset{v}{\bigoplus} r P_{j_{v}}^{m_{j v}{ }^{v}}$, segue que $S_{l}$ é módulo de Koszul, pois $S_{l}$ é um módulo gerado em grau zero.

\section{8 Álgebras de Koszul inclinadas}

Nesta seção apresentaremos nosso principal resultado, em decorrência da introdução do conceito de aplicações poço de torção, que nos permitiu 
caracterizar as álgebras inclinadas graduadas que são álgebras de Koszul, descrever uma subaljava ordinária destas álgebras e ainda, descrever os módulos de torção que definem módulos de Koszul sobre esta álgebra inclinada.

Sendo o resultado de caráter mais geral, do que aquele que tomamos inicialmente, em nosso trabalho, estaremos prontos para decidir se as álgebras dadas por anéis de endomorfismos de módulos inclinantes sobre $k$-álgebras de dimensão finita, cujo ideal da apresentação da álgebra seja graduado, são Koszul ou não, investigando as aplicações envolvidas na $\Gamma$-resolução projetiva de $\Gamma / r$.

Sabemos por [AS, 4 e 5], que as álgebras inclinadas generalizadas de tipo $A_{n}$, são quadráticas monomiais. O nosso resultado fornece uma demonstração diagramática deste fato e que nos possibilita concluir que estas álgebras são álgebras de Koszul, assim como descrever a aljava e relações destas álgebras.

Estaremos mantendo a notação usada ao longo deste capítulo e a que foi usada no parágrafo anterior.

Sejam $\Lambda$ uma $k$-álgebra de dimensão finita, sobre um corpo algebricamente fechado $k$, e $T$ um $\Lambda$-módulo inclinante, graduado, gerado em grau zero e livre de multiplicidade, dado por $T=\bigoplus_{l=1}^{n} T_{l}$, com $T_{l}$ um $\Lambda$-módulo indecomponível. Seja $\Gamma=\operatorname{End}_{\Lambda}(T)^{o p}$, o anel de endomorfismo de $T$ sobre $\Lambda$, tal que $\Gamma \cong k Q / I$, onde $Q$ é uma aljava finita e $I$ é um ideal admissível.

Definição 4.16 Com as condições fixadas acima, seja $l \in\{1, \cdots, n\}$ tal que $r P_{l} \neq 0$. Dizemos que o $\Lambda$-módulo $E_{l} \in \mathcal{T}(T)$ é um predecessor de torção do $\Lambda$-módulo $T_{l}$, quando ocorrer que $r P_{l} \cong \operatorname{Hom}_{\Lambda}\left(T, E_{l}\right)$.

Daqui por diante, denotaremos por $E_{l} \circ \Lambda$-módulo de torção tal que o morfismo $E_{l} \stackrel{\alpha}{\longrightarrow} T_{l}$ é o poço de torção minimal, para cada $T_{l}$, somando direto indecomponível do inclinante $T, \operatorname{com} r P_{l} \neq 0$. Lembremos que chamamos $E_{l}$ de predecessor de torção de $T_{l}$.

Definição 4.17 Consideremos $\Gamma$ nas condições fixadas acima. Seja $E$ um $\Gamma$-módulo de torção. Dizemos que um par $\left(T_{E}, \phi\right)$, onde $T_{E}$ é um $\Lambda$-módulo e $\phi: T_{E} \longrightarrow E$ é um morfismo, é um gerador de torção de $E$, se satisfaz as seguintes propriedades:

(i) $T_{E} \in$ add $T$ e $\phi: T_{E} \longrightarrow E$ é um epimorfismo. 
(ii) todo epimorfismo $\psi: T^{\prime} \longrightarrow E$, onde $T^{\prime} \in$ add $T$ se fatora através de $\phi$.

Dizemos que $\left(T_{E}, \phi\right)$ é um gerador minimal se $\phi$ é morfismo minimal à direita, (cf Def. 4.10).

Lema 4.18 Dado $E \in \mathcal{T}(T)$, existe um único gerador de torção minimal, $\left(T_{E}, \phi\right)$ para E. Além disso, $\operatorname{Hom}_{\Lambda}\left(T, T_{E}\right)$ é a cobertura projetiva de $\operatorname{Hom}_{\Lambda}(T, E)$.

Demonstração: Se ocorre que $(T, E)$ é um projetivo, então temos que o par $\left(E, 1_{E}\right)$ é um gerador de torção minimal para $E$. Caso contrário, consideremos a cobertura projetiva $\pi_{*}:\left(T, T^{\prime}\right) \longrightarrow(T, E)$, onde $T^{\prime} \in$ add $T$. Como o funtou $T \otimes$ - é exato à direita, resulta que o morfismo $\pi: T^{\prime} \longrightarrow E$, que induz $\pi_{*}$, é um epimorfismo. Desde que $\pi_{*}$ é minimal, segue que $\pi$ é minimal. Queremos mostrar que o par $\left(T^{\prime}, \pi\right)$ minimal é único.

Seja $\left(T^{\prime \prime}, \phi\right)$ gerador de torção minimal de $E$. Pela definição de gerador de torção, temos que $\pi$ se fatora através de $\phi$, ou seja, existe $\alpha: T^{\prime} \longrightarrow T^{\prime \prime}$, tal que $\pi=\phi \alpha$.

Por outro lado, o morfismo $\phi$ induz o $\Gamma$-morfismo $\phi_{*}:\left(T, T^{\prime \prime}\right) \longrightarrow(T, E)$ que se fatora por $\pi_{*}$, ou seja, existe $\beta_{*}:\left(T, T^{\prime \prime}\right) \longrightarrow\left(T, T^{\prime}\right)$ tal que $\phi_{*}=\pi_{*} \beta_{*}$. Assim, obtemos que $\pi_{*}=\pi_{*}(\beta \alpha)_{*}$, resultando que $(\beta \alpha)_{*}$ é um ismorfismo. Pela BB-equivalência, segue que $T^{\prime \prime} \cong T^{\prime}$.

Teorema 4.19 Sejam $\Lambda$ uma k-álgebra de dimensão finita, $T$ um $\Lambda$-módulo inclinante e $\Gamma=\operatorname{End}_{\Lambda}(T) \cong k Q /_{I}$, onde $Q$ é uma aljava finita, $I$ é um ideal graduado. Seja $r=L / I$, o radical graduado de Jacobson de $\Gamma$, onde $L$ é o ideal de $k Q$ gerado pelas flechas. Considere a gr $(\Gamma)$-resolução projetiva minimal de $\Gamma / r$

$$
\cdots \longrightarrow P_{(2)} \stackrel{f_{*}}{\longrightarrow} P_{(1)} \longrightarrow \Gamma \longrightarrow \Gamma / r \rightarrow 0
$$

onde $P_{(j)}=\operatorname{Hom}_{\Lambda}\left(T, T_{j}^{\prime}\right)$, para cada $j \geq 1$, com $T_{j}^{\prime} \in$ add $T$. Seja $E_{j} a$ soma direta dos predecessores de torção de cada um dos somandos diretos indecomponíveis de $T_{j}^{\prime}$, com $j \geq 1$. Então, $\Gamma$ é uma álgebra de Koszul se e somente se $T_{j+1}^{\prime}$ é somando direto do gerador de $E_{j}$, para cada $j \geq 1$. 
Demonstração: Suponhamos que $\Gamma$ seja uma álgebra de Koszul. Então, para cada $j, P_{(j)}$ é gerado em grau $j$, ou equivalentemente, $P_{(j)}$ é somando direto de cobertura projetiva de $r P_{(j-1)}$.

Sejam $E_{j} \in \mathcal{T}(T)$ tal que $\operatorname{Hom}_{\Lambda}\left(T, E_{j}\right) \cong r P_{(j)}$ e $T^{\prime}$ o gerador de torção de $E_{j}$. Então, pelo Lema 4.18 , temos que $\operatorname{Hom}_{\Lambda}\left(T, T^{\prime}\right)$ é a cobertura projetiva de $r P_{(j)}$. Segue que $P_{(j+1)}$ é um somando direto de $\operatorname{Hom}_{\Lambda}\left(T, T^{\prime}\right)$ e, pela BBequivalência aplicada aos módulos de torção, teremos que, para cada $j \geq 1, T_{j}^{\prime}$ é somando direto de $T^{\prime}$.

Reciprocamente, se $T_{j+1}^{\prime}$ é um somando direto de $T^{\prime}$, o gerador de torção minimal de $E_{j}$, então $P_{(j+1)}$ é um somando direto da cobertura projetiva de $r P_{(j)}$, para cada $j \geq 1$. Como $\Gamma$ é 1 -gerada, resulta que $P_{(j+1)}$ é gerado em grau $j+1$, (cf. capítulo 1). Logo, $\Gamma$ é Koszul.

Com os resultados que já apresentamos, podemos descrever um grafo orientado subjacente à aljava ordinária de $\Gamma$, desde que se $r P_{l} \neq 0$ então $\left(T, T^{\prime}\right)=P_{(0)}\left(T, E_{l}\right)=P_{(0)}\left(r P_{l}\right)$, onde $T^{\prime}$ é o gerador de torção de $E_{l}$. Considere $T_{l_{1}, l}, \ldots, T_{l_{n}, l}$ os somandos diretos indecomponíveis, não-isomorfos, de $T^{\prime}$. Então, o grafo orientado abaixo, é um esboço do comportamento local de uma subaljava de $Q(\Gamma)$ :

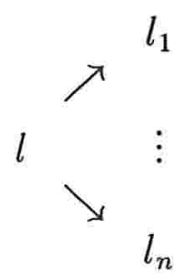

\subsection{Exemplos}

Neste parágrafo, vamos aplicar nossos resultados a algumas álgebras particulares, ao caso das classes de álgebras BB-inclinadas e das de tipo $A_{n}$ (cf. cap. 3 e $[\mathrm{AS}, 4])$. 


\section{9 .1}

Considere $\Lambda$ a $k$-álgebra dada por:

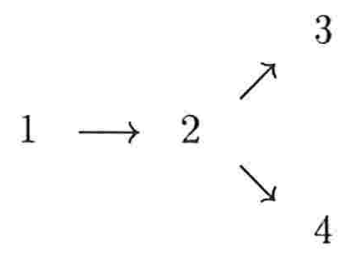

Seja $T=\tau^{-} S_{2} \oplus P_{1} \oplus P_{3} \oplus P_{4}, \Lambda$-módulo inclinante. A aljava de A-R de $\Lambda$ é dado pelo seguinte grafo:

$\left(P_{3}\right)$

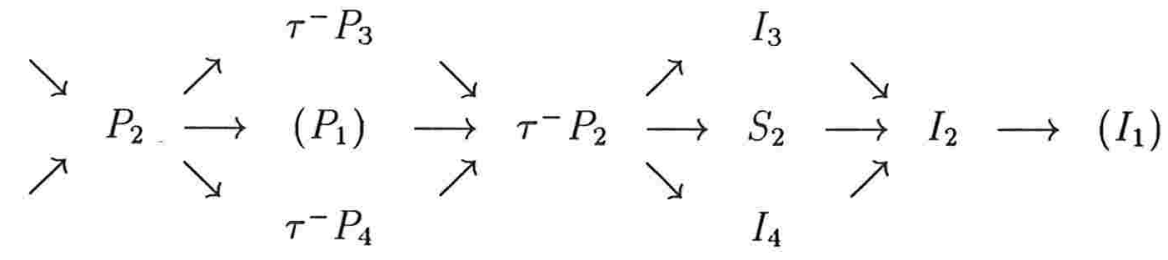

onde os módulos entre parênteses são os somandos diretos indecomponíveis de $T$, e as flechas representam morfismos irredutíveis.

Temos que o morfismo $\left(P_{2} \longrightarrow P_{1}\right)$, representado no grafo acima, é o morfismo minimal quase cindido à direita, minimal, com contradomínio em $P_{1}$; mais ainda, temos que $P_{3} \oplus P_{4}$ é o gerador de torção $\operatorname{de~} \operatorname{tr}_{T} P_{2}$. Também, temos que o gerador de torção de $I_{2}$ é $P_{1}$ (observando que o topo de $I_{2}$ é $S_{1}$ ), e que $I_{2} \longrightarrow \tau^{-} S_{2}=I_{1}$ é o morfismo que induz o morfismo quase cindido à direita, minimal, dado pela inclusão $r\left(T, \tau^{-} S_{2}\right) \hookrightarrow\left(T, \tau^{-} S_{2}\right)$.

Temos para $Q(\Gamma)$, o seguinte grafo:

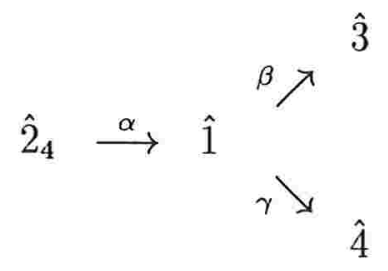

Neste caso, como temos que $\left(P_{j}, \tau^{-} S_{2}\right)=0$, para $j=3,4$, segue que $\Gamma$ é dada pela aljava acima, com as relações $\beta \alpha=0=\gamma \alpha$. Observamos que, neste exemplo, as noções de poço torção minimal e de gerador de torção minimal foram suficientes para construir a aljava de $\Gamma$. 


\subsection{2}

Considere a $k$-álgebra dada por:

$$
1 \longleftarrow 2 \longleftarrow 3 \longleftarrow{ }_{5}^{4}
$$

Seja $T=\tau^{-3} P_{1} \oplus \tau^{-2} P_{5} \oplus \tau^{-2} P_{3} \oplus \tau^{-2} P_{2} \oplus \tau^{-1} P_{4}$ um $\Lambda$-módulo inclinante.

Um esboço local da aljava de $A R$ de $\Lambda$ é dado por:

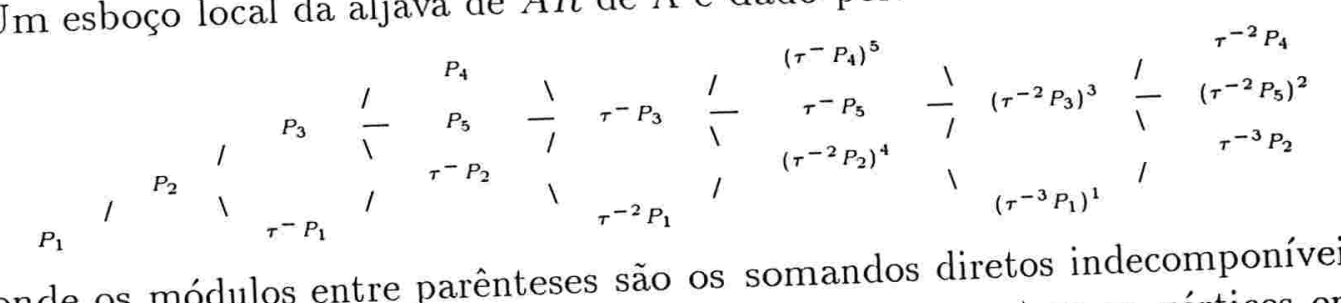

onde os módulos entre parênteses são os somandos diretos indecomponíveis de $T$, os números que indexam os parênteses representam os vértices em $Q(\Gamma)$ aos quais estes somandos estão associados, e os traços entre os módulos representam morfismos irredutíveis da esquerda para a direita.

Temos que a subaljava da aljava de $A R$ de $\Lambda$ definida pelos somandos diretos indecomponíveis de $T$, é dada pelo seguinte grafo:

$$
\begin{array}{cccc}
\tau^{-} P_{4}^{(5)} & & \\
& \searrow & \\
& & \tau^{-2} P_{3}^{(3)} & \longrightarrow \\
& \nearrow & \\
\tau^{-2} P_{2}^{(4)} & & \\
& \searrow & \\
& & \tau^{-3} P_{1}^{(1)}
\end{array}
$$

onde as flechas são morfismos irredutíveis e os únicos caminhos que ligam estes somandos, logo são os morfismos que induzem as aplicações poço de torção. Pelo corolário 4.13, concluimos que $\Gamma$ tem radical projetivo.

Con efeito, temos que $\Gamma=\operatorname{End}_{\Lambda}(T)^{o p}$ é hereditária com $Q(\Gamma)$ dada por:

$$
1 \longrightarrow 4 \longleftarrow 3 \longleftarrow 2
$$




\section{9 .3}

Considere $\Lambda$ a $k$-álgebra dada por:

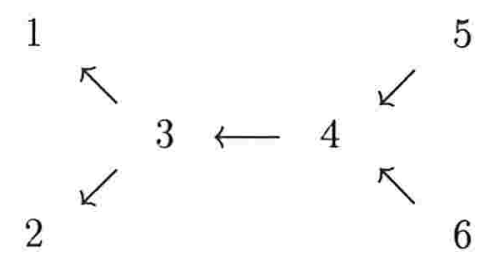

Temos o seguinte esboço local da componente preprojetiva da aljava de $A R$ de $\Lambda$ :

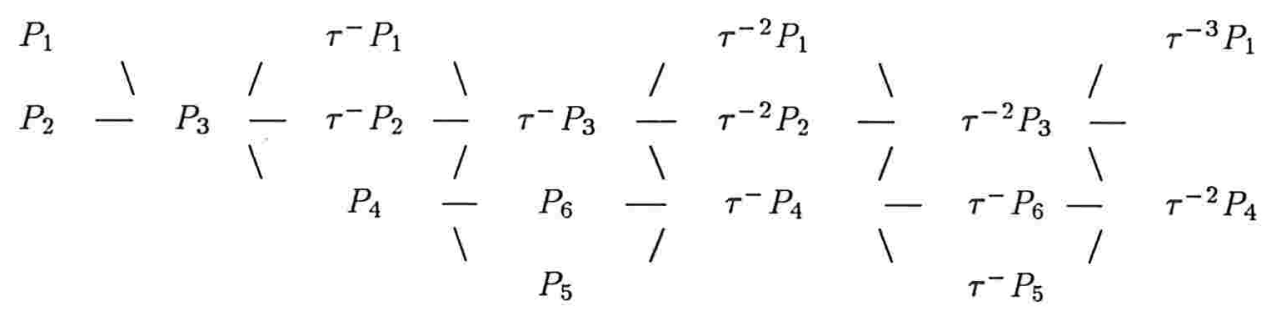

onde os traços representam os morfismos irredutíveis entre os módulos indecomponíveis, considerados da esquerda para a direita.

Considere o $\Lambda$-módulo inclinante dado por:

$$
T=\tau^{-} P_{5} \oplus \tau^{-} P_{4} \oplus \tau^{-} P_{3} \oplus \tau^{-} P_{2} \oplus \tau^{-} P_{1} \oplus P_{6}
$$

Temos que os somandos diretos indecomponíveis de $T$ formam a seguinte subaljava da aljava de $A R$ de $\Lambda$ :

$$
\begin{aligned}
& \tau^{-} P_{1} \\
& \tau^{-} P_{2} \longrightarrow \tau^{-} P_{3} \\
& P_{6} \longrightarrow \tau^{-} P_{4} \\
& \tau^{-} P_{5}
\end{aligned}
$$

onde as flechas são morfismos irredutíveis. Segue que $\Gamma$ tem radical projetivo. 
Com efeito, temos a aljava de $\Gamma$ dada por:

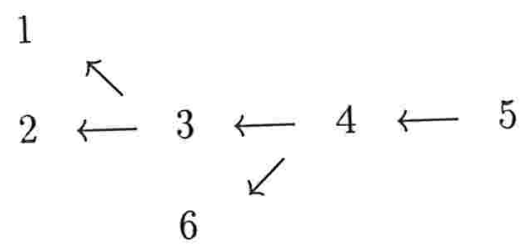

sem relações, pois $\Gamma$ é hereditária.

\subsection{4}

Seja $\Lambda$ a álgebra de caminhos de uma aljava $Q$, cujo grafo subjacente é $A_{n}$. Dizemos que uma $k$-álgebra de dimensão finita $B$ é uma álgebra inclinada generalizada do tipo $A_{n}$, quando existir uma família de álgebras $A_{i}$ e uma família de módulos inclinante $T_{i}={ }_{A_{i}} T, \operatorname{com} A_{0}=\Lambda, A_{i+1}=\operatorname{End}_{A_{i}}(T)^{o p}$ e a teoria de torção $\left(\mathcal{X}\left({ }_{A_{i}} T\right), \mathcal{Y}\left({ }_{A_{i}} T\right)\right)$ cinde para $i>0$, tal que $B=A_{m}$ para algum $m \in \mathbb{N}$.

Em $[\mathrm{AS}, 4]$, I. Assem provou que as álgebras inclinadas dadas por módulos inclinantes sobre $\Lambda$, são quadráticas monomiais. Em [AS,5], foram dadas várias condições que juntas formam uma condição necessária e suficiente para que uma álgebra seja inclinada generalizada do tipo $A_{n}$. Uma destas condições é o fato de que, estas álgebras, são quadráticas monomiais.

Usando nosso resultado e um lema apresentado em [AS,5], podemos concluir esta assertiva, assim como descvever a aljava ordinária destas álgebras, assim como foi feito neste trabalho de I. Assem. Mais ainda, podemos concluir que a classe das álgebras que satisfazem a propriedade enunciada neste lema, vai produzir uma álgebra quadrática monomial. Vejamos como isto ocorre.

Seja $\Lambda$ uma $k$-álgebra de dimensão finita, cuja aljava de Auslander-Reiten, $\Gamma_{A}$, satisfaz as seguintes propriedades:

(1) $\Gamma_{A}$ é simplesmente conexa.

(2) Existem no máximo dois morfismos irredutíveis para cada domínio ou contradomínio pré-fixado. 
(3) Se $P$ é um $\Lambda$-módulo projetivo com radical, $r P$, indecomponível, então existe no máximo um morfismo irredutível com contradomínio $r P$.

Lema [AS,5]: Seja $A$ a $k$-álgebra de dimensão finita e tipo de representação finito que satisfaz as propriedades acima. Então, para cada $A$-módulo indecomponível $M$, temos que o conjunto dos $A$-módulos indecomponíveis $N$, (classes de isomorfismos), tal que existe uma aplicação não-nula $N \longrightarrow M$, mas, não existe uma aplicação não-nula $N \longrightarrow \tau M$, é uma união de duas subaljavas plenas lineares de $\Gamma_{A}$, interceptando em [M].

Dualmente, o conjunto de todos os $A$-módulos indecomponíveis $L$, (classes de isomorfismos), tal que existe uma aplicação não-nula $M \longrightarrow L$, mas, não existe uma aplicação não-nula $\tau^{-} M \longrightarrow L$, é a união de duas subaljavas lineares plenas em $\Gamma_{A}$, interceptando em [M].

Demonstração: cf. em [AS,5].

Observamos que uma aljava é linear se para cada vértice desta aljava, existe, no máximo, uma flecha saindo e uma flecha chegando neste vértice. Decorrente do lema enunciado acima, temos que dado $T_{l}$ somando direto indecomponível do $A$-módulo inclinante $T$, existem, no máximo, dois morfismos irredutíveis chegando em $T_{l}$ e no máximo, dois saindo de $T_{l}$, sendo que estes morfismos determinam, no máximo, 4 subaljavas lineares interceptando em $T_{l}$; e tal que todo módulo indecomponível em $\Gamma_{A}$ que não pertença à estas subaljavas não possuem morfismos não-nulos chegando em $T_{l}$, ou não são contradomínios de morfismos não nulos saindo de $T_{l}$.

$\mathrm{O}$ desenho a seguir pode ajudar a fixar as idéias envolvidas no lema. Sejam $f_{1}, f_{2}$ morfismos irredutíveis chegando em $T_{l}$ e sejam $g_{1}, g_{2}$ os que saem. Sejam $L_{1}, L_{2}$ e $R_{1}, R_{2}$ as subaljavas lineares à esquerda e à direita de $T_{l}$, respectivamente, se interceptando em $T_{l}$. Podemos considerar o seguinte 
grafo orientado

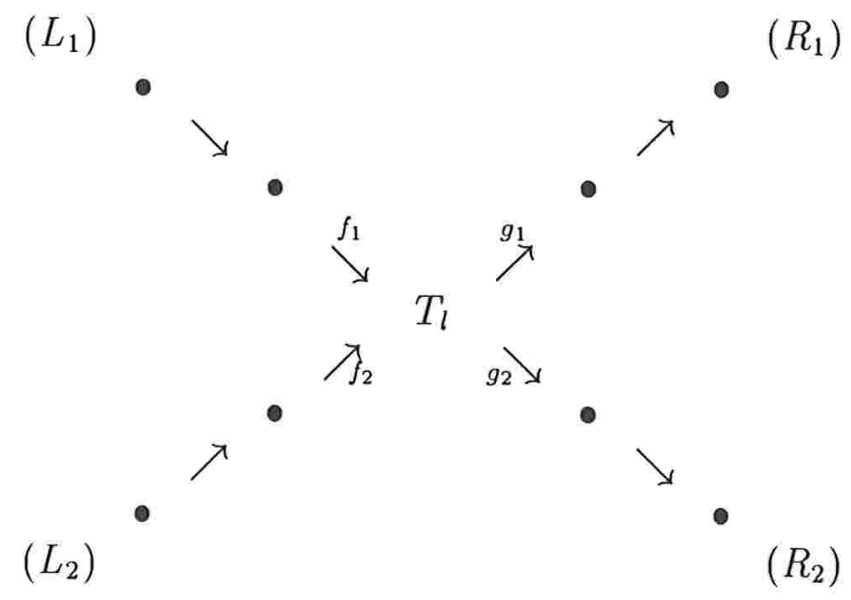

Por nossas hipóteses, teremos que o predecessor de torção $E_{l}$ do indecomponível $T_{l}$, pertence à $L_{1} \cup L_{2}$, caso seja $r P_{l} \neq 0$, e também, teremos que $E_{l}$ tem, no máximo, dois somandos diretos indecomponíveis. Aplicando o lema para cada um destes somandos, concluiremos que o gerador de torção de $E_{l}$ tem, no máximo, quatro somandos diretos indecomponíveis, não-isomorfos, em add $T$. Consideremos $E_{l}=E_{l_{1}} \oplus E_{l_{2}}$, com $E_{l_{j}}$ indecomponível, para cada $j=1,2$. Obteremos, então, o seguinte grafo orientado:

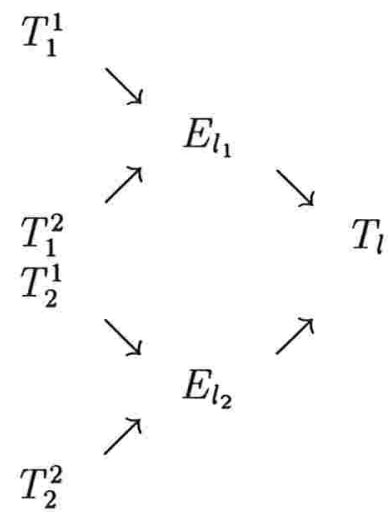

onde $T_{i}^{j}$ é somando direto indecomponível do gerador de $E_{l}$, para cada $i, j$, e tal que $T_{i}^{i}$ pertence a subaljava $L_{i}, \operatorname{com} i=1,2$.

Como $T_{1}^{2}$ não pertence à $L_{1}$ ( e, análogamente $T_{2}^{1}$ não pertence à $L_{2}$ ) segue que $\operatorname{Hom}_{A}\left(T_{1}^{2}, T_{l}\right)=0\left(\mathrm{e}\right.$, respectivamente, $\left.\operatorname{Hom}_{A}\left(T_{2}^{1}, T_{l}\right)=0\right)$. Assim, teremos que os morfismos entre os módulos $T_{l}$ e $T_{1}^{1}$, e entre $T_{l}$ e $T_{2}^{2}$, induzem 
as componentes da cobertura projetiva de $r P_{l}$. Portanto, existem flechas do vértice $l$ aos vértices associados aos projetivos $\operatorname{Hom}_{A}\left(T, T_{1}^{1}\right)$ e $\operatorname{Hom}_{A}\left(T, T_{2}^{2}\right)$. Como estes são o únicos morfismos não nulos possíveis, chegando em $T_{l}$, segue que definem as únicas flechas que podem existir de $l$ para estes vértices.

Para conveniência de notação, vamos reescrever $T_{1}^{1}=T_{1}$ e $T_{2}^{2}=T_{2}$. Aplicando a argumentação que acabamos de fazer para o módulo $T_{l}$, a cada um dos módulos indecomponíveis $T_{1}$ e $T_{2}$, definidos acima, obteremos, primeiramente, um diagrama análogo ao dado acima, bastando trocar $E_{l_{i}}$ por $T_{i}$, para cada $i=1,2$. Em seguida, podemos concluir que os módulos que não pertencem às subaljavas lineares do tipo $L_{i}, i=1,2$, e que se interceptam nos módulos $T_{1}$ e $T_{2}$, não possuem morfismos não nulos chegando neste módulos.

Vamos refazer nosso diagrama, para maior clareza de nossas idéias. Consideremos, agora, $T_{1}^{j} \operatorname{com} j,=1,2$, os somandos diretos indecomponíveis do gerador de torção do predecessor de torção de $T_{1}$, que possuem morfismos não nulos chegando em $T_{1}$, e, análogamente, $T_{2}^{j}$ para $T_{2}$. Então, temos o seguinte grafo:

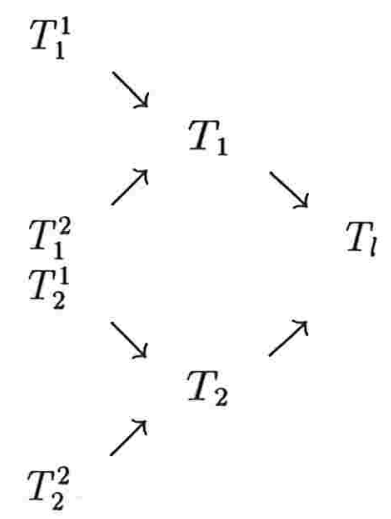

Portanto, se os módulos $T_{i}^{j} \operatorname{com} i \neq j$, definidos acima, são não nulos, teremos uma relação em $\Gamma$, saindo do vértice $l$ e chegando nos vértices associados aos projetivos indecomponíveis $\left(T, T_{1}^{2}\right)$ e $\left(T, T_{2}^{1}\right)$. Observamos que $T_{1}^{2} \not$ $T_{2}^{1}$, pois, caso contrário, existiria uma relação de comutatividade iniciando no vértice $l$, e envolvendo os projetivos indecomponíveis $P_{l},\left(T, T_{1}\right),\left(T, T_{2}\right)$ e $\left(T, T_{1}^{2}\right) \cong\left(T, T_{2}^{1}\right)$, implicando a existência de um morfismo não-nulo de $T_{1}^{2}$ para $T_{l}$. Portanto, teríamos que ter $T_{1}^{2} \in L_{1}$, uma contradição.

Com argumentos análogos aos apresentados acima, podemos concluir que: 
(i) Se $T_{l}$ tem 2 predecessores e 2 sucessores em add $T$, então $Q(\Gamma)$ contém uma subaljava dada por:

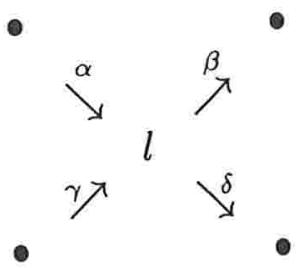

$\operatorname{com} \beta \alpha=0=\delta \gamma$.

(ii) Se $T_{l}$ tem 2 predecessores e um sucessor em add $T$, então $Q(\Gamma)$ contém subaljava dada por:

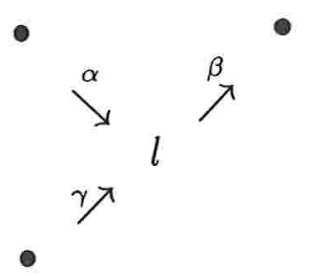

$\operatorname{com} \beta \alpha=0$

Análogamente, se ocorrem 1 predecessor e 2 sucessores tem-se uma subaljava dada por:

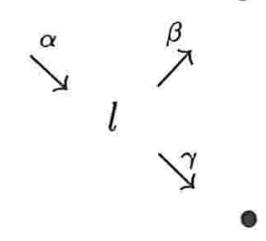

$\operatorname{com} \beta \alpha=0$.

Observamos que não pode ocorrer $\beta \gamma=0 \mathrm{em}$ (I), ou $\gamma \alpha=0 \mathrm{em}$ (II), pois, necessáriamente os módulos, somandos diretos indecomponíveis de $T$, que determinam os projetivos associados aos vértices envolvidos nestes caminhos, pertencem a mesma subaljava linear $L_{2}$ e $L_{1}$, respectivamente, não determinando, portanto, caminhos nulos na aljava de $\Gamma$. 


\subsection{As classes $\mathcal{L}(\Gamma)$ e $\mathcal{K}(\Gamma)$}

Nosso interesse neste parágrafo é fornecer alguns exemplos envolvendo álgebras hereditárias, as BB-inclinadas e um relato do que ocorre sobre as álgebras de tipo $A_{n}$ e as inclinadas generalizadas de tipo $A_{n}$, no que se refere aos módulos com apresentação linear e aos módulos de Koszul destas álgebras.

De acordo com [GMRSZ], sabemos que se $\Gamma$ é graduada gerada em graus zero e um então $\mathcal{L}(\Gamma) \cong \mathcal{L}\left(\Gamma / r^{2}\right)$. No caso das hereditárias temos que todo módulo sobre $\Gamma / r^{2}$ é um módulo de Koszul, já que $\Gamma / r^{2}-\bmod \cong \mathcal{K}\left(\Gamma / r^{2}\right) \cong$ $\mathcal{L}\left(\Gamma / r^{2}\right) \cong \mathcal{L}(\Gamma) \cong \mathcal{K}(\Gamma)$.

Assim, encontrar os módulos com apresentação linear, neste caso, equivale a encontrar os módulos sobre $\Gamma / r^{2}$. Temos por $[\mathrm{AP}]$, que $\Gamma / r^{2}$ é estávelmente equivalente a álgebra definida por:

$$
\left(\begin{array}{cc}
\Gamma / r & 0 \\
r & \Gamma / r
\end{array}\right)
$$

cuja aljava é dada pela aljava separada de $\Gamma$ (cf. definição na pag. 350, em [ARS]. Assim, podemos calcular estas classes com certa facilidade. No caso de álgebras inclinadas graduadas $\Gamma=\operatorname{End}_{\Lambda}(T)^{o p}$, com ideal de relações homogêneo, teremos que $\mathcal{L}(\Gamma) \cong \mathcal{L}\left(\Gamma / r^{2}\right)$. Vejamos como estes fatos funcionam.

Exemplo 1. Sabemos que, sobre álgebras graduadas quadráticas monomiais $\Gamma$, as classes $\mathcal{L}(\Gamma)$ e $\mathcal{K}(\Gamma)$ dos módulos com apresentação linear e dos módulos de Koszul, respectivamente, coincidem (cf. [GMRSZ]). Segue que sobre as álgebras de tipo $A_{n}$ e as inclinadas generalizadas de tipo $A_{n}$, os módulos de Koszul são exatamente os que possuem apresentação linear.

Exemplo 2. Seja $\Lambda=k \tilde{D}_{5}$, com a seguinte orientação em sua aljava ordinária:

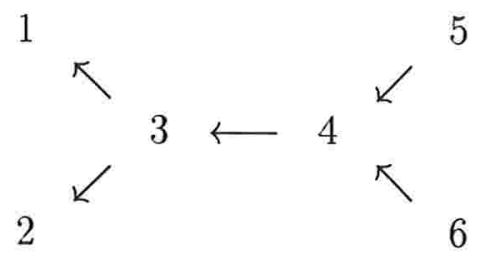


Temos que o grafo separado, estavelmente equivalente à $\Lambda / r^{2}$, é dado por $A_{3} \times A_{2} \times A_{3}$, que possui 13 módulos indecomponíveis, não-isomorfos. Segue que $\Lambda$ possui 13 módulos, não isomorfos, com apresentação linear. Como $\Lambda$ é hereditária, todos eles são módulos de Koszul.

Exemplo 2. Seja $\Lambda=k \tilde{D}_{5}$ como no exemplo 1 e tomemos $\Gamma=\Gamma_{4}$, a álgebra BB-inclinada associada ao vértice 4.

Ou seja, $\Gamma=\operatorname{End}_{\Lambda}(T)^{o p}, \operatorname{com} T=\tau^{-} S_{4} \oplus \underset{\substack{j=1 \\ j \neq 4}}{\bigoplus} P_{j}$, onde $P_{j}$ é projetivo indecomponivel.

Temos que $\Gamma$ é dada pela aljava:

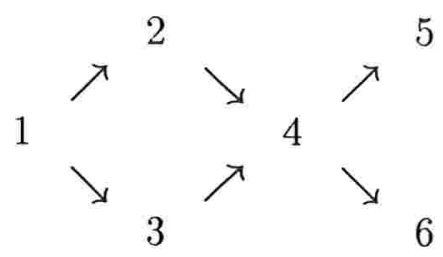

com a relação de comutatividade no quadrado. Temos que o grafo separado de $\Gamma / r^{2}$ é dado por: $A_{3} \times A_{3} \times A_{3}$.

Segue que $\Gamma$ tem 15 módulos, não isomorfos, com apresentação linear. Como $\hat{S}_{1}$, o $\Gamma$-módulo simples associado ao vértice 1 é um dos módulos de $\Gamma$ com apresentação linear (na verdade, é um módulo de Koszul), temos que todos os outros serão módulos de Koszul, pois pertencem a $\mathcal{Y}(T)$. Mais ainda, nesta situação temos que $\mathcal{L}(\Gamma) \cong \mathcal{K}(\Gamma)$, como já vimos no cap. 3 , seção 5 . No exemplo 1 vimos que existe sobre $\Lambda$, apenas 13 classes de isomorfia de módulos de Koszul, indecomponíveis. Ou seja, além de não ser verdade que módulos de Koszul sobre $\Lambda$ produzem $\Gamma$-módulos de Koszul como foi mostrado na seção 3.5 , também, temos que as classes $\mathcal{K}(\Lambda)$ e $\mathcal{K}(\Gamma)$ não se correspondem numericamente, mesmo sendo finitas. 


\section{Bibliografia}

[AP] Auslander, M.; Platzeck, M.I. Representation theory of hereditary artin algebras. Lectures Notes in Pure and Applied Math., 37, Marcel Dekker, New York and Basel (1978), 389-424.

[ARS] Auslander, M.; Reiten, I.; Smalo, S.Ø. Representation theory of Artin algebras. Cambridge Studies in Advanced Mathematics, 36.

[AS1] Assem, I. Tilting Theory. Publicação da Unversité de Sherbrooke, Sherbroke, Québec, Canada; (1988).

[AS2] Assem, I.; Skownonski, A. Tilting simply connected algebras. Comm. in Algebra, 22 (12), 4611-4619 (1994).

[AS3] Assem, I. Torsion theories induced by tilting modules. Canad. J. math., vol. XXXVI, no -5, (1984), pp. 899-913.

[AS4] Assem, I. Tilted Algebras of type $A_{n}$. Comm. in Alg., 10(19), 2121-2139 (1982).

[AS5] Assem, I.; Happel D. Generalized tilted algebras of type $A_{n}$. Comm. in Alg., 9 (20), 2101-2125 (1981).

[BB] Brenner, S.; Butler, M. Generalizations of Bernstein-Gelfand-Ponomarev reflection functors. Proc. ICRA II (Ottawa, 1979), LNM, 832 Springer-Verlag, Berlin (1980), 103-169.

[B] Bongartz, K. Algebras and quadratic forms. J. London Math. Soc. (2), 28 (1983), 461-469.

[FGGM] Farkas, D.; Geiss, C.; Green, E.L.; Marcos, E.N. Diagonalizable derivations and graduations, em preparação. 
[GG] Gordon, R.; Green, E.L. Graded Artin Algebras. Journal of Algebra, 76, 111-137 (1982).

[GHM] Goze, M.; Hakimjanov, Yu. ; Makhlouf, A. Sur les algèbres associatives caractèristiquement nilpotentes. Comm. in Algebra, 22(8), 2961-2968 (1994).

[GM1] Green, E.L.; Martinez-Villa, R. Koszul and Yoneda algebras. Canadian Math. Soc., 18, (1994), 247-298.

[GM2] Green, E.L.; Martinez-Villa, R. Koszul and Yoneda algebras II. Canadian Math. Soc., 24, (1996), 227-244.

[GMRSZ] Green, E.L.; Martinez-Villa, R.; Reiten, I.; Solberg, Ø; Zacharia, D. On modules with linear presentations, em preparação.

[HR] Happel, D.; Ringel, C.M. Construction of tilted algebras. LNM, 903, Springer-Verlag, 125-142.

[HR1] Happel, D.; Ringel, C.M. Tilted algebras. Amer. Math. Soc., (1982), 399-443.

[HV] Happel, D.; Vossieck, D. Minimal algebras of infinite representation type with preprojective component. Manuscripta Math., 42, 221-243 (1983).

[R] Ringel, C.M. Tame algebra. LNM, 1099, Springer-Verlag, Berlin. 